FIRST QUARTER 1993

\title{
K-AREA ACID/CAUSTIC BASIN GROUNDWATER MONITORING REPORT (U)
}

\section{KEY WORDS}

aluminum

iron

lead

KAC wells

specific conductance

PUBLICATION DATE: JUNE 1993

WESTINGHOUSE SAVANNAH RIVER COMPANY SAVANNAH RIVER SITE AIKEN, SC 29808

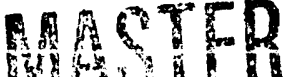

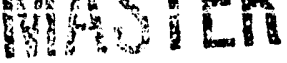


2

$-\cdots$

WSRC-TR-93-277 


\section{Abstract}

During first quarter 1993, samples from the KAC monitoring wells at the K-Area Acid/Caustic Basin were analyzed for indicator parameters, groundwater quality parameters, parameters indicating suitability as drinking water, and other constituents. Wells KAC 8 and 9 also were sampled for GC/MS VOA (gas chromatograph/mass spectrometer volatile organic analyses). Monitoring results that exceeded the final Primary Drinking Water Standards (PDWS) or the Savannah River Site (SRS) flagging criteria or turbidity standard during the quarter are discussed in this report.

Aluminum exceeded its Flag 2 criterion in wells $\mathrm{KAC} 6,7,8$, and 9. Iron exceeded the Flag 2 criterion in wells $\mathrm{KAC} 6,7$, and 8, lead was elevated in well $\mathrm{KAC} 7$, and specific conductance exceeded the Flag 2 criterion in well KAC 9. No samples exceeded the SRS turbidity standard. 
WSRC-TR-93-277 


\section{Contents}

Page

Abstract $\ldots \ldots \ldots \ldots \ldots \ldots \ldots \ldots \ldots \ldots \ldots \ldots \ldots \ldots \ldots \ldots \ldots \ldots \ldots \ldots$ iii

List of Figures $\ldots \ldots \ldots \ldots \ldots \ldots \ldots \ldots \ldots \ldots \ldots \ldots \ldots \ldots \ldots \ldots \ldots \ldots$

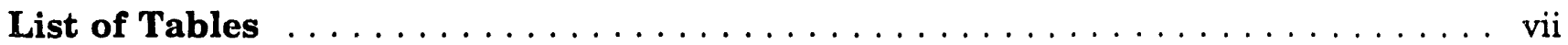

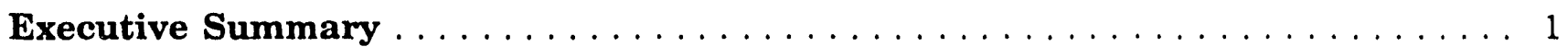

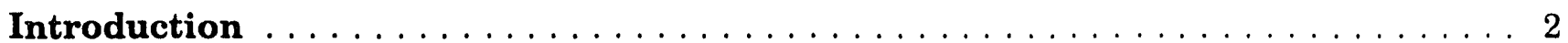

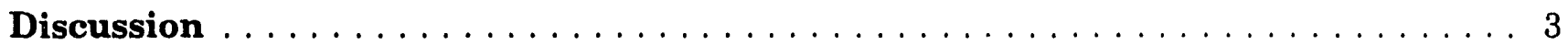

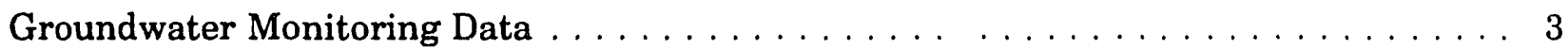

Analytical Results Exceeding Standards $\ldots \ldots \ldots \ldots \ldots \ldots \ldots \ldots \ldots \ldots$

Turbidity Results Exceeding Standards $\ldots \ldots \ldots \ldots \ldots \ldots \ldots \ldots \ldots \ldots$

Water Elevations, Flow Directions, and Flow Rates $\ldots \ldots \ldots \ldots \ldots \ldots \ldots \ldots \ldots$

Results for Upgradient vs. Downgradient Wells $\ldots \ldots \ldots \ldots \ldots \ldots \ldots \ldots \ldots \ldots 6$

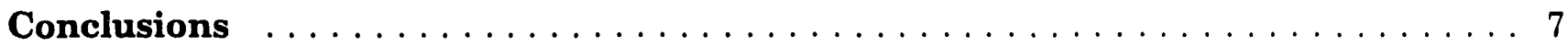

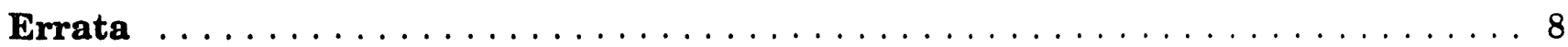

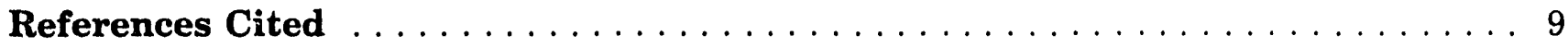

Appendix A-Final Primary Drinking Water Standards $\ldots \ldots \ldots \ldots \ldots \ldots$ A-1

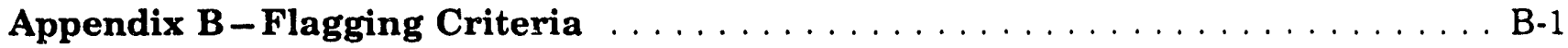

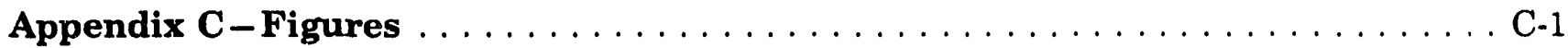

Appendix D-Groundwater Monitoring Results Tables $\ldots \ldots \ldots \ldots \ldots \ldots$ D-1

Appendix E-Data Quality/Useability Assessment $\ldots \ldots \ldots \ldots \ldots \ldots \ldots$ E-1 


\section{List of Figures}

Page

1. Location of the K-Area Acid/Caustic Basin at the Savannah River Site . . . . . . . C-2

2. Location of Groundwater Monitoring Wells at the K-Area Acid/Caustic Basin . . . . C C-3

3. Water-Elevation Contour Map of the Water Table at the K-Area Acid/Caustic

Basin .......................................

C-4

\section{List of Tables}

Page

1. Maximum Results for Constituents Exceeding Final Primary Drinking Water

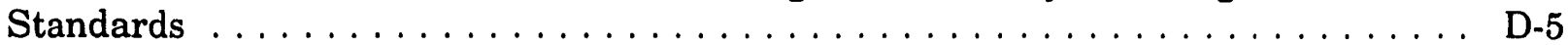

2. Maximum Results for Constituents Exceeding Half their Final Primary Drinking Water Standards, Other Flag 1 or Flag 2 Criteria, or the SRS Turbidity Standard

3. Groundwater Monitoring Results for Individual Wells . . . . . . . . . . . . . . D $\quad$-6 
WSRC-TR-93-277 
WSRC-TR-93-277

\section{Executive Summary}

The monitoring wells at the K-Area Acid/Caustic Basin are sampled quarterly as part of the Savannah River Site (SRS) Groundwater Monitoring Program and to comply with the terms of a consent decree signed May 26, 1988, by the U.S. District Court (District of South Carolina, Aiken Division). During first quarter 1993, samples from the monitoring wells were analyzed for indicator parameters, groundwater quality parameters, parameters indicating suitability as drinking water, and other constituents. Monitoring results that exceeded final Primary Drinking Water Standards (PDWS), other SRS flagging criteria, or the SRS turbidity standard are discussed in this report.

During first quarter 1993, no constituents exceeded the final PDWS. Aluminum exceeded its Flag 2 criterion in four wells, iron exceeded the Flag 2 criterion in three wells, lead was elevated in well KAC 7, and specific conductance exceeded the Flag 2 criterion in KAC 9. No samples exceeded the SRS turbidity standard. 


\section{Introduction}

The K-Area Acid/Caustic Basin is located in the eastern portion of K Area at the Savannah River Site (SRS) near a tributary of Pen Branch. The basin, constructed in the early 1950s, is an unlined earthen pit that received dilute sulfuric acid and sodium hydroxide solutions and other wastes from several areas within SRS. The basin provided an area for the mixing and neutralization of the dilute solutions before their discharge to nearby streams. The $\mathrm{K}$-Area Acid/Caustic Basin remained in service until new neutralization facilities became operational in 1982 (Heffner and Exploration Resources, 1991).

Four groundwater monitoring wells were installed at the K-Area Acid/Caustic Basin between October 1983 and July 1984. Under the terms of a consent decree signed May 26, 1988, by the U.S. District Court (District of South Carolina, Aiken Division), on June 1, 1988, the basin became subject tu requirements of Subtitle $\mathrm{C}$ of the Resource Conservation and Recovery Act (RCRA), the South Carolina Hazardous Waste Management Regulations (SCHWMR), and associated regulations. The basin monitoring wells were reevaluated during the summer of 1988 to ensure compliance with SCHWMR. As part of this compliance effort, three additional wells were installed at the K-Area Acid/Caustic Basin in the fall of 1988.

The monitoring wells at the K-Area Acid/Caustic Basin are sampled quarterly as part of the SRS Groundwater Monitoring Program and to comply with the consent decree. The revised Groundwater Quality Assessment Plan (WSRC, 1991), submitted to the South Carolina Department of Health and Environmental Control on April 30, 1991, proposed the installation of two additional water-table wells at the K-Area Acid/Caustic Basin. These wells, KAC 8 and 9, have been installed and were first sampled during second quarter 1992. 
WSRC-TR-93-277

\section{Discussion}

\section{Groundwater Monitoring Data}

The groundwater sampling procedure was modified beginning fourth quarter 1992 in response to regulatory guidance and advances in sampling equipment design (WSRC, 1992). The modified procedure requires evacuation of a minimum of two well volumes and stabilization of $\mathrm{pH}$, specific conductance, and turbidity prior to sample collection. Stability is established when a minimum of three successive measurements, taken within a given time period, are within a specified tolerance range. If a well pumps dry before two well volumes are purged or before stabilization is achieved, it must be revisited within 24 hours for the data to be considered from a single sampling event. On the second visit within 24 hours, samples are taken without purging or stability measurements; thus, these samples may not be representative of the groundwater quality.

A further modification in the procedure is that samples collected for metals analyses are not filtered. Thus, the analyses are for total metals rather than dissolved metals. In addition, variable-speed pumps have been installed in some wells in specific areas that have had a history of elevated metals. Samples from these wells are collected at a slower rate to minimize turbidity, which has been associated with elevated metal levels. Decreased aluminum and iron concentrations as well as lower turbidity values have been observed for samples from wells with variable-speed pumps. Well KAC 1 has a variable-speed pump.

During first quarter 1993, samples from the monitoring wells at the K-Area Acid/Caustic Basin were analyzed for indicator parameters, groundwater quality parameters, parameters indicating suitability as drinking water, and other constituents. Wells KAC 8 and 9 also were sampled for GC/MS VOA (gas chromatograph/mass spectrometer volatile organics analyses).

This report describes monitoring results that exceeded the Safe Drinking Water Act final Primary Drinking Water Standards (PDWS) set by the U.S. Environmental Protection Agency (EPA) (Appendix A), the South Carolina final PDWS for lead (Appendix A), other SRS flagging criteria (Appendix B), or the SRS turbidity standard. Constituent levels that equal or exceed the final PDWS, screening levels, or Flag 2 criteria are described as elevated, and constituent levels that equal or exceed Flag 1 criteria are described as slightly elevated.

The S.RS flagging criteria generally are based on final and proposed PDWS, Secondary Drinking Water Standards, and method detection limits. The final PDWS for individual analytes provided in Appendix A may not always match the SRS flagging criteria provided in Appendix B. The final PDWS are used as guidelines in this compliance report to meet regulatory requirements; the flagging criteria are used by the Environmental Protection Department/Environmental Monitoring Section to identify relative levels of constituents in the groundwater and as guides for scheduling groundwater sampling. 
Illustrations of the monitored unit at SRS (Figure 1), the individual monitoring wells (Figure 2), and the flow directions of the groundwater beneath the unit (Figure 3) are in Appendix C; monitoring results as well as analyses that exceeded holding times, the final PDWS, other flagging criteria, or the turbidity standard are presented in Appendix D; and a discussion of data quality and useability is in Appendix E.

\section{Analytical Results Exceeding Standards}

Results for any analytes that exceeded the final PDWS (see Appendix A) during first quarter 1993 are summarized in Table 1 (Appendix D); there were none.

Constituents that exceeded other Flag 1 or Flag 2 criteria (see Appendix B) during first quarter 1993 are summarized in Table 2 (Appendix D). Aluminum exceeded its Flag 2 criterion in wells $\mathrm{KAC} 6,7,8$, and 9 , with a maximum concentration of $4,660 \mu \mathrm{g} / \mathrm{L}$. Iron exceeded the Flag 2 criterion in wells $\operatorname{KAC~} 6,7$, and 8 , with a maximum concentration of $987 \mu \mathrm{g} / \mathrm{L}$. All of the iron values above standard are qualified with the modifier $V$, indicating that iron also was detected in the associated blank(s). Lead exceeded its Flag 2 criterion in well KAC 7 with a concentration of $42 \mu \mathrm{g} / \mathrm{L}$. Specific conductance exceeded the Flag 2 criterion in well $\mathrm{KAC} 9$ at $924 \mu \mathrm{S} / \mathrm{cm}$.

Table 3 (Appendix D) presents all of the results for individual wells and indicates those analyses that exceeded holding times or the final PDWS. Table 3 also lists the number of well volumes purged from each well during first quarter 1993 at the K-Area Acid/Caustic Basin. Wells KAC 6 and 7 each failed to yield enough water to meet the purging and stabilization criteria. They were sampled within 24 hours. The sampler noted that the water from well KAC 7 was tan.

Constituent results are compared with the PDWS in the database of values reported by the laboratory. Many constituents are reported to more significant digits in the database than in these reports. Thus, some constituent results in Table 3 that appear to equal the PDWS are not marked in the $D$ column. Those results are below the PDWS in the database.

Some of the values for earlier quarters presented in Table 1 of this report may differ from the values for those same quarters presented in previous reports, and reported values may not match reported sample dates. These differences result from the following: (1) a new computer program, which rounds numbers differently from the former computer program, was first used during third quarter 1992; (2) some reanalyses may have been performed by the laboratories after the quarterly reports had gone to press; and (3) the sample dates in the tables are the dates when the field data were collected. These dates may differ from the dates of the laboratory analyses if the highest results were obtained for samples collected on different dates. 


\section{Turbidity Results Exceeding Standards}

A value of 5 nephelometric turbidity units (NTU), established by EPA (1986) as a general standard for acceptability of groundwater samples, is considered unrealistic for monitoring wells at SRS. Gass (1989) has documented turbidity measurements ranging up to 5,000 NTU from properly designed wells screened in poorly productive formations. During the 1989 RCRA Compliance Evaluation Inspection, officials from EPA Region IV indicated that the SRS turbidity standard of $50 \mathrm{NTU}$ is conservative. These officials also agreed that watertable wells in this area often correspond to nonaquifer formations, rendering development of these wells more difficult due to the low yield and high proportion of mobile fines typical of these formations (Bergren and Bennett, 1989).

During first quarter 1993, wells $\mathrm{KAC} 2,6,7$, and 8 had tu kidity values between $5 \mathrm{NTU}$ and the SRS standard of 50 NTU.

\section{Water Elevations, Flow Directions, and Flow Rates}

Water-table elevations and the groundwater flow direction beneath the K-Area Acid/Caustic Basin are shown in Figure 3 (Appendix C). Historical data indicate a ponding or losing stream effect (water entering the groundwater from the K-Area outfall effluent stream) in this area, creating a mound of groundwater to the east of the basin. Water-table elevations from this quarter indicate that the groundwater flow direction is northwest (using SRS grid coordinates). This is consistent with information from fourth quarter 1992, when wells KAC 8 and 9 were first included in the determination.

However, determinations of groundwater flow direction made over the past several years without wells KAC 8 and 9 consistently indicated a direction of southwest or west-southwest.

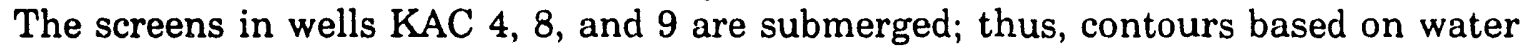
elevations from these wells might not accurately depict water-table conditions.

The groundwater flow rate in the water table beneath the K-Area Acid/Caustic Basin is estimated using the following equation:

$$
\text { Flow }(\mathrm{ft} / \text { day })=\frac{\text { Hydraulic Conductivity }(\mathrm{ft} / \text { day })}{\text { Porosity (unitless) }} \times \frac{d h(\mathrm{ft} ;}{d l(\mathrm{ft})}
$$

A hydraulic conductivity constant of $4 \mathrm{ft} /$ day is based on recent hydrogeological studies for this location (Atlanta Testing and Engineering, 1992). The effective porosity value is estimated at $20 \%$ (Killian et al., 1987), $d h$ is the difference in head, and $d l$ is the length of the flow path to the nearest foot. Flow rate estimates vary depending on the vertical gradient between wells, the size of the area under consideration, and the number of data points. For this reason, the estimation of flow rate must be considered accurate only to an order of inagnitude.

Flow rate estimates are calculated as follows: flow rate per day is calculated to two significant figures using the above equation. This value is then multiplied by 365 and rounded to two significant figures for the flow rate per year. 
Using the above equation with $d h=9 \mathrm{ft}$ and $d l=156 \mathrm{ft}$ (see Figure 3, Appendix C), the flow rate estimate for groundwater in the water table beneath the K-Area Acid/Caustic Basin is as follows:

$$
\frac{4}{0.20} \times \frac{9}{156}=1.2 \mathrm{ft} / \mathrm{day}
$$

$$
1.2 \mathrm{ft} / \text { day } \times 365 \text { days } \approx 440 \mathrm{ft} / \mathrm{yr}
$$

Flow rate estimates have varied considerably during recent quarters, as shown below.

$\begin{array}{ccccc}\cdot & \underline{2 Q 92} & \underline{3 Q 92} & \underline{4 Q 92} & \underline{1 Q 93} \\ \text { Groundwater flow rate (ft/yr) } & 330 & 160 & 800 & 440\end{array}$

\section{Results for Upgradient vs. Downgradient Wells}

Wells KAC 3 and 5 are the upgradient wells. No constituents exceeded the final PDWS, other Flag 2 criteria, or the SRS turbidity standard in these wells. 
WSRC-TR-93-277

\section{Conclusions}

No constituents exceeded the PDWS at the K-Area Acid/Caustic Basin during first quarter 1993. Aluminum exceeded the Flag 2 criterion in downgradients wells $\operatorname{KAC~6,~7,~8,~and~9;~}$ iron exceeded the Flag 2 criterion in wells KAC 6, 7, and 8, lead was above its Flag 2 standard in well KAC 7, and specific conductance exceeded the Flag 2 criterion in well KAC 9. Generally, constituents found in downgradient wells but not upgradient wells at a waste management unit are considered products of the waste management unit.

Wells KAC 2, 6, 7, and 8 had turbidity values between 5 and 50 NTU. Wells KAC 6 and 7 did not yield two well volumes prior to sampling.

Water-table elevations at the K-Area Acid/Caustic Basin indicate that groundwater flow is toward the northwest relative to SRS grid coordinates. The screens in wells $\mathrm{KAC} 4,8$, and 9 are submerged and may provide anomalous water-level data. 


\section{Errata}

Second Quarter 1992:

- No errata have been reported.

Third Quarter 1992:

- Prior to third quarter 1992 , the results of certain analyses for nitrate-nitrite as nitrogen were reported incorrectly by the General Engineering laboratory as nitrate as nitrogen results. The analyses in the results tables beginning this quarter are reported correctly (nitrate-nitrite results have been separated from true nitrate results).

Fourth Quarter 1992:

- No errata have been reported. 
WSRC-TR-93-277

\section{References Cited}

Atlanta Testing \& Engineering, 1992. Hydrogeology of the F-Area and K-Area Acid Caustic Basins, Savannah River Site, WSRC-RP-91504. Westinghouse Savannah River Company, Aiken, SC.

Bergren, C. L., and C. B. Bennett, 1989. Assessment of SRS Groundwater Monitoring Wells Impacted by Turbidity, WSRC-RP-89-891. Westinghouse Savannah River Company, Aiken, SC.

EPA (U.S. Environmental Protection Agency), 1986. RCRA Ground Water Monitoring Technical Enforcement Guidance Document, OSWER-9950.1. Washington, DC.

Gass, T. E., 1989. Monitoring Wells in Non-Aquifer Formations. Water Well Journal 43(2):27-29.

Heffner, J. D., and Exploration Resources, Inc., 1991. Technical Summary of Groundwater Quality Protection Program at the Savannah River Site (1952-1986), Volume I-Site Geohydrology and Waste Sites, DPSP-88-1002. Westinghouse Savannah River Company, Aiken, SC.

Killian, T. H., N. L. Kolb, P. Corbo, and I. W. Marine, 1987. F-Area Seepage

Basins, DPST-85-704. Savannah River Laboratory, E. I. du Pont de Nemours \& Company, Aiken, SC.

WSRC (Westinghouse Savannah River Company), 1991. F-, H-, K-, and P-Area Acid/

Caustic Basins Groundwater Quality Assessment Plan, WSRC-TR-91-178, Revision 1.0. Westinghouse Savannah River Company, Aiken, SC.

WSRC, 1992. Hydrogeologic Data Collection Procedures and Specifications: Sampling Groundwater Monitoring Wells, Manual 3Q5, Chapter 14, Revision 0. Environmental Protection Department, Environmental Monitoring Section, Savannah River Site, Aiken, SC. 
WSRC-TR-93-277 
WSRC-TR-93-277

Appendix A - Final Primary Drinking Water Standards 


\section{Final Primary Drinking Water Standards}

\begin{tabular}{|c|c|c|c|c|}
\hline Analyte & $\underline{\text { Unit }}$ & Level & $\underline{\text { Status }}$ & Reference \\
\hline Antimony & $\mu \mathrm{g} / \mathrm{L}$ & 6 & Final & EPA, 1992b \\
\hline Arsenic & $\mu \mathrm{g} / \mathrm{L}$ & 50 & Final & EPA, $1992 a$ \\
\hline Asbestos & fibers/L & $7,000,000$ & Final & EPA, 1992a \\
\hline Barium & $\mu g / L$ & 2,000 & Final & EPA, 1992a \\
\hline Benzene & $\mu \mathrm{g} / \mathrm{L}$ & 5 & Final & EPA, 1992a \\
\hline Benzolalpyrene & $\mu g / L$ & 0.2 & Final & EPA, $1992 b$ \\
\hline Beryllium & $\mu \mathrm{g} / \mathrm{L}$ & 4 & Final & EPA, $1992 b$ \\
\hline Bis(2-ethylhexyl) phthalate & $\mu \mathrm{g} / \mathrm{L}$ & 6 & Final & EPA, 1992b \\
\hline Bromodichloromethane & $\mu g / L$ & $100^{a}$ & Final & EPA, 1992a \\
\hline Bromoform & $\mu \mathrm{g} / \mathrm{L}$ & $100^{a}$ & Final & EPA, 1992a \\
\hline 2-sec-Butyl-4,6-dinitrophenol & $\mu g / L$ & 7 & Final & $E P A, 1992 b$ \\
\hline Cadmium & $\mu g / L$ & 5 & Final & EPA, $1992 a$ \\
\hline Carbon tetrachloride & $\mu \mathrm{g} / \mathrm{L}$ & 5 & Final & EPA, 1992a \\
\hline Chlordane & $\mu \mathrm{g} / \mathrm{L}$ & 2 & Final & $E P A, 1992 a$ \\
\hline Chlorobenzene & $\mu \mathrm{g} / \mathrm{L}$ & 100 & Final & EPA, 1992a \\
\hline Chloroethene (Vinyl chloride) & $\mu \mathrm{g} / \mathrm{L}$ & 2 & Final & EPA, 1992a \\
\hline Chloroform & $\mu g / L$ & $100^{a}$ & Final & EPA, $1992 a$ \\
\hline Chromium & $\mu \mathrm{g} / \mathrm{L}$ & 100 & Final & EPA, $1992 a$ \\
\hline Copper & $\mu g / L$ & 1,300 & Final & EPA, $1992 a$ \\
\hline Cyanide & $\mu \mathrm{g} / \mathrm{L}$ & 200 & Final & EPA, $1992 b$ \\
\hline Dibromochloromethane & $\mu \mathrm{g} / \mathrm{L}$ & $100^{a}$ & Final & EPA, $1992 a$ \\
\hline Dibromochloropropane & $\mu g / L$ & 0.2 & Final & EPA, $1992 a$ \\
\hline 1,2-Dibromoethane (Ethylene dibromide) & $\mu \mathrm{g} / \mathrm{L}$ & 0.05 & Final & EPA, 1992a \\
\hline 1,2-Dichlorobenzene & $\mu \mathrm{g} / \mathrm{L}$ & 600 & Final & EPA, $1992 a$ \\
\hline 1,4-Dichlorobenzene & $\mu \mathrm{g} / \mathrm{L}$ & 75 & Final & EPA, $1992 a$ \\
\hline 1,2-Dichloroethane & $\mu \mathrm{g} / \mathrm{L}$ & 5 & Final & $E P A, 1992 a$ \\
\hline 1,1 -Dichloroethene & $\mu \mathrm{g} / \mathrm{L}$ & 7 & Final & EPA, 1992a \\
\hline 1,2 -Dichloroethene & $\mu \mathrm{g} / \mathrm{L}$ & 50 & Final & EPA, $1992 b$ \\
\hline cis-1,2-Dichloroethene & $\mu g / L$ & 70 & Final & $E P A, 1992 a$ \\
\hline trans-1,2-Dichloroethene & $\mu \mathrm{g} / \mathrm{L}$ & 100 & Final & $E P A, 1992 a$ \\
\hline Dichloromethane (Methylene chloride) & $\mu \mathrm{g} / \mathrm{L}$ & 5 & Final & EPA, $1992 b$ \\
\hline 2,4-Dichlorophenoxyacetic acid & $\mu \mathrm{g} / \mathrm{L}$ & 70 & Final & $E P A, 1992 a$ \\
\hline 1,2-Dichloropropane & $\mu \mathrm{g} / \mathrm{L}$ & 5 & Final & EPA, 1992a \\
\hline Endrin & $\mu g / L$ & 2 & Final & EPA, $1992 b$ \\
\hline Ethylbenzene & $\mu \mathrm{g} / \mathrm{L}$ & 700 & Final & $E P A, 1992 a$ \\
\hline $\begin{array}{l}\text { Fluoride } \\
\end{array}$ & $\mu \mathrm{g} / \mathrm{L}$ & 4,000 & Final & $E P A, 1992 a$ \\
\hline Gross alphab & $\mathrm{pCi} / \mathrm{L}$ & $1.5 \mathrm{E}+01$ & Final & EPA, $1992 a$ \\
\hline Heptachlor & $\mu g / L$ & 0.4 & Final & EPA, 1992a \\
\hline Heptachlor epoxide & $\mu \mathrm{g} / \mathrm{L}$ & 0.2 & Final & EPA, 1992a \\
\hline Hexachlorobenzene & $\mu \mathrm{g} / \mathrm{L}$ & 1 & Final & $E P A, 1992 b$ \\
\hline Hexachlorocyclopentadiene & $\mu \mathrm{g} / \mathrm{L}$ & 50 & Final & EPA, 1992b \\
\hline Lead & $\mu \mathrm{g} / \mathrm{L}$ & 50 & Final & SCDHEC, 1981 \\
\hline Lindane & $\mu \mathrm{g} / \mathrm{L}$ & 0.2 & Final & $E P A, 1992 a$ \\
\hline Mercury & $\mu \mathrm{g} / \mathrm{L}$ & 2 & Final & $E P A, 1992 a$ \\
\hline Methoxychlor & $\mu \mathrm{g} / \mathrm{L}$ & 40 & Final & $E P A, 1992 a$ \\
\hline Nickel & $\mu g / L$ & 100 & Final & $E P A, 1992 b$ \\
\hline Nitrate as nitrogen & $\mu \mathrm{g} / \mathrm{L}$ & 10,000 & Final & $E P A, 1992 a$ \\
\hline Nitrate-nitrite as nitrogen & $\mu \mathrm{g} / \mathrm{L}$ & 10,000 & Final & EPA, 1992a \\
\hline Nitrite as nitrogen & $\mu \mathrm{g} / \mathrm{L}$ & 1,000 & Final & EPA, 1992a \\
\hline Nonvolatile beta ${ }^{c}$ & $\mathrm{pCi} / \mathrm{L}$ & $5 E+01$ & Final & EPA, 1977 \\
\hline PCBs ${ }^{d}$ & $\mu \mathrm{g} / \mathrm{L}$ & 0.5 & Final & EPA, $1992 a$ \\
\hline Pentachlorophenol & $\mu \mathrm{g} / \mathrm{L}$ & 1 & Final & EPA, $1992 a$ \\
\hline Selenium & $\mu \mathrm{g} / \mathrm{L}$ & 50 & Final & $E P A, 1992 a$ \\
\hline
\end{tabular}


Analyte

Strontium-89/90

Strontium-90

Styrene

2,3,7,8-TCDD

Tetrachloroethylene

Thallium

Toluene

Total radium (Radium-226 and -228)

Total trihalomethanes

Toxaphene

2,4,5-TP (Silvex)

1,2,4-Trichlorobenzene

1,1,1-Trichloroethane

1,1,2-Trichloroethane

Trichloroethylene

Tritium

Xylenes
Unit

$\mathrm{pCi} / \mathrm{L}$

$\mathrm{pCi} / \mathrm{L}$

$\mu \mathrm{g} / \mathrm{L}$

$\mu \mathrm{g} / \mathrm{L}$

$\mu \mathrm{g} / \mathrm{L}$

$\mu \mathrm{g} / \mathrm{L}$

$\mu \mathrm{g} / \mathrm{L}$

$\mathrm{pCi} / \mathrm{L}$

$\mu g / L$

$\mu \mathrm{g} / \mathrm{L}$

$\mu g / L$

$\mu g / L$

$\mu \mathrm{g} / \mathrm{L}$

$\mu \mathrm{g} / \mathrm{L}$

$\mu \mathrm{g} / \mathrm{L}$

$\mathrm{pCi} / \mathrm{mL}$

$\mu \mathrm{g} / \mathrm{L}$
Level

$8 E+00$

$8 \mathrm{E}+00$

100

0.00003

5

2

1,000

$5 \mathrm{E}+00$

100

3

50

70

200

5

5

$2 \mathrm{E}+01$

10,000 $\underline{\text { Status }}$

Final

Final

Final

Final

Final

Final

Final

Final

Final

Final

Final

Final

Final

Final

Final

Final

Final
Reference

EPA, 1992a

EPA, 1992a

EPA, $1992 a$

EPA, 1992b

EPA, 1992a

EPA, 1992b

EPA, 1992a

EPA， 1992a

EPA，1992a

EPA, 1992a

EPA, 1992a

EPA, 1992b

EPA, $1992 a$

EPA, 1992b

EPA, 1992a

EPA, 1992a

EPA, 1992a

a This value is the drinking water standard for total trihalomethanes (the sum of bromoform, bromodichloromethane, chloroform, and dibromochloromethane).

b The standard given is for gross alpha including radium-226 but excluding radon and uranium.

c This is the screening level above which providers of public drinking water should perform analyses for specific man-made radionuclides. The standard for the total dose equivalent from all such radionuclides is 4 mrem per year.

d Analyses were conducted in 1992 for the following: PCB 1016, PCB 1221, PCB 1232, PCB 1242, PCB 1248, PCB 1254, and PCB 1260.

- For double radionuclide analyses where each separate radionuclide has its own standard, the more stringent standard is used.

\section{References}

EPA (U.S. Environmental Protection Agency), 1977. National Interim Primary Drinking Water Regulations, EPA570/9-76-003. Washington, DC.

EPA (U.S. Environmental Protection Agency), 1992a. National Primary Drinking Water Regulations, Code of Federal Regulations, Section 40, Part 141, pp. 589-729. Washington, DC.

EPA (U.S. Environmental Protection Agency), 1992b. National Primary Drinking Water Regulations - Synthetic Organic Chemical and Inorganic Chemicals; National Primary Drinking Water Regulations Implementation. Federal Register, July 17, 1992, pp. 31776-31849. Washington, DC.

SCDHEC. (South Carolina Department of Health and Environmental Control), 1981. State Primary Drinking Water Regulations, R.61-58.5. Columbia, SC. 
WSRC-TR-93-277 
WSRC-TR-93-277

Appendix B - Flagging Criteria 


\section{Flagging Criteria}

The Savannah River Site Environmental Protection Department/Environmental Monitoring Section (EPD/EMS) flagging criteria are as follows:

- Flag 2 criteria for constituents equal the Safe Drinking Water Act (SDWA) final Primary Drinking Water Standard (PDWS), the SDWA proposed PDWS, or the SDWA Secondary Drinking Water Standard (SDWS). If a constituent does not have a drinking water standard, the Flag 2 criterion equals 10 times the method detection limit (MDL) calculated as the 90 th percentile detection limit obtained recently by one of the primary analytical laboratories.

- Flag 1 criteria for constituents equal one-half of the final PDWS, one-half the proposed PDWS, or one-half the SDWS. If a constituent does not have a drinking water standard, the Flag 1 criterion equals 5 times the MDL calculated as the 90th percentile detection limit obtained recently by one of the primary analytical laboratories.

- Flag 0 criteria are assigned to constituent levels below Flag 1 criteria, constituent levels below the sample detection limits, or constituents having no flagging criteria.

The following parameters are not assigned flagging criteria: alkalinity, calcium, color, corrosivity, Eh, magnesium, odor, potassium, silica, sodium, total dissolved solids, total phosphates (as P), total phosphorus, and turbidity. In addition, common laboratory contaminants and cleaners including some phthalates, ketones, and toluene are not assigned flagging criteria.

\begin{tabular}{|c|c|c|c|c|}
\hline Analyte & Unit & Flag 1 & Flag 2 & Source $^{a}$ \\
\hline Acenaphthene & $\mu \mathrm{g} / \mathrm{L}$ & 50 & 100 & EPA Method 8270 \\
\hline Acenaphthylene & $\mu g / L$ & 50 & 100 & EPA Method 8270 \\
\hline Acetone & $\mu \mathrm{g} / \mathrm{L}$ & 500 & 1,000 & EPA Method 8240 \\
\hline Acetonitrile (Methyl cyanide) & $\mu \mathrm{g} / \mathrm{L}$ & 500 & 1,000 & EPA Method 8240 \\
\hline Acetophenone & $\mu \mathrm{g} / \mathrm{L}$ & 50 & 100 & EPA Method 8270 \\
\hline 2-Acetylaminofluorene & $\mu g / L$ & 50 & 100 & EPA Method 8270 \\
\hline Acrolein & $\mu \mathrm{g} / \mathrm{L}$ & 100 & 200 & EPA Method 8240 \\
\hline Acrylonitrile & $\mu \mathrm{g} / \mathrm{L}$ & 100 & 200 & EPA Method 8240 \\
\hline Aldrin & $\mu g / L$ & 0.25 & 0.5 & EPA Method 8080 \\
\hline Alkalinity (as $\mathrm{CaCO}_{3}$ ) & & No flag & No flag & Set by EPD/EMS \\
\hline Allyl chloride & $\mu \mathrm{g} i$ & 250 & 500 & EPA Method 8240 \\
\hline Aluminum & $\mu \mathrm{g} / \mathrm{L}$ & 25 & 50 & SDWS (EPA, 1992c) \\
\hline Arnericium-241 & $\mathrm{pCi} / \mathrm{L}$ & $3.17 E+00$ & $6.34 E+00$ & Proposeci PDWS (EPA, 1991) \\
\hline Americium-243 & $\mathrm{pCi} / \mathrm{L}$ & $3.19 E+00$ & $6.37 E+00$ & Proposed PDWS (EPA, 1991) \\
\hline 4-Aminobiphenyl & $\mu g / L$ & 50 & 100 & EPA Method 8270 \\
\hline Arnmonia & $\mu \mathrm{g} / \mathrm{L}$ & 500 & 1,000 & APHA Method 417B \\
\hline Ammonia nitrogen & $\mu \mathrm{g} / \mathrm{L}$ & 500 & 1,000 & EPA Method 350.1 \\
\hline Aniline & $\mu \mathrm{g} / \mathrm{L}$ & 50 & 100 & EPA Method 8270 \\
\hline Anthracene & $\mu \mathrm{g} / \mathrm{L}$ & 50 & 100 & EPA Method 8270 \\
\hline Antimony & $\mu g / L$ & 3 & 6 & Final PDWS (EPA, 1992b) \\
\hline Antimony-125 & $\mathrm{pCi} / \mathrm{L}$ & $1.5 E+02$ & $3 E+02$ & Final PDWS (EPA, 1977) \\
\hline Aramite & $\mu \mathrm{g} / \mathrm{L}$ & 50 & 100 & EPA Method 8270 \\
\hline Arsenic & $\mu \mathrm{g} / \mathrm{L}$ & 25 & 50 & Final PDWS (EPA, 1992a) \\
\hline Asbestos & Fibers/L & $3,500,000$ & $7,000,000$ & Final PDWS (EPA, 1992a) \\
\hline
\end{tabular}


WSRC-TR-93-277

\begin{tabular}{|c|c|c|c|c|}
\hline Analyte & $\underline{\text { Unit }}$ & Flag 1 & Flag 2 & Source $^{a}$ \\
\hline Azobenzene & $\mu \mathrm{g} / \mathrm{L}$ & 50 & 100 & EPA Method 625 \\
\hline Barium & $\mu \mathrm{g} / \mathrm{L}$ & 1,000 & 2,000 & Final PDWS (EPA, 1992a) \\
\hline Barium-140 & $\mathrm{pCi} / \mathrm{L}$ & $4.5 E+01$ & $9 E+01$ & Final PDWS (EPA, 1977) \\
\hline Benzene & $\mu g / L$ & 2.5 & 5 & Final PDWS (EPA, 1992a) \\
\hline alpha-Benzene hexachloride & $\mu g / L$ & 0.25 & 0.5 & EPA Method 8080 \\
\hline beta-Benzene hexachloride & $\mu g / L$ & 0.25 & 0.5 & EPA Method 8080 \\
\hline delta-Benzene hexachloride & $\mu \mathrm{g} / \mathrm{L}$ & 0.25 & 0.5 & EPA Method 8080 \\
\hline Benzidine & $\mu g / L$ & 250 & 500 & EPA Method 8270 \\
\hline Benzola]anthracene & $\mu \mathrm{g} / \mathrm{L}$ & 0.05 & 0.1 & Proposed PDWS (EPA, 1990) \\
\hline Benzolb]fluoranthene & $\mu \mathrm{g} / \mathrm{L}$ & 0.1 & 0.2 & Proposed PDWS (EPA, 1990) \\
\hline Benzolk]fluoranthene & $\mu \mathrm{g} / \mathrm{L}$ & 0.1 & 0.2 & Proposed PDWS (EPA, 1990) \\
\hline Benzoic acid & $\mu \mathrm{g} / \mathrm{L}$ & 250 & 500 & EPA Method 8270 \\
\hline Benzolg,h,i]perylene & $\mu g / L$ & 50 & 100 & EPA Method 8270 \\
\hline Benzola]pyrene & $\mu \mathrm{g} / \mathrm{L}$ & 0.1 & 0.2 & Final PDWS (EPA, 1992b) \\
\hline 1,4-Benzoquinone & $\mu \mathrm{g} / \mathrm{L}$ & 50 & 100 & EPA Method 8270 \\
\hline Benzyl alcohol & $\mu g / L$ & 50 & 100 & EPA Method 8270 \\
\hline Beryllium & $\mu \mathrm{g} / \mathrm{L}$ & 2 & 4 & Final PDWS (EPA, 1992b) \\
\hline Beryllium-7 & $\mathrm{pCi} / \mathrm{L}$ & $3 E+03$ & $6 E+03$ & Final PDWS (EPA, 1977 ) \\
\hline Bis(2-chloroethoxy) methane & $\mu \mathrm{g} / \mathrm{L}$ & 50 & 100 & EPA Method 8270 \\
\hline Bis (2-chloroethyl) ether & $\mu g / L$ & 50 & 100 & EPA Method 8270 \\
\hline Bis (2-chloroisopropyl) ether & $\mu \mathrm{g} / \mathrm{L}$ & 50 & 100 & EPA Method 8270 \\
\hline Bis (chloromethyl) ether & $\mu \mathrm{g} / \mathrm{L}$ & 50 & 100 & EPA Method 8270 \\
\hline Bis (2-ethylhexyl) phthalate & $\mu g / L$ & 3 & 6 & Final PDWS (EPA, 1992b) \\
\hline Bromide & $\mu \mathrm{g} / \mathrm{L}$ & 5,000 & 10,000 & EPA Method 300.0 \\
\hline Bromodichloromethane & $\mu \mathrm{g} / \mathrm{L}$ & 50 & 100 & Final PDWS (EPA, 1992a) \\
\hline Bromoform & $\mu g / L$ & 50 & 100 & Final PDWS (EPA, 1992a) \\
\hline Bromomethane (Methyl bromide) & $\mu \mathrm{g} / \mathrm{L}$ & 5 & 10 & EPA Method 8240 \\
\hline 4-Bromophenyl phenyl ether & $\mu g / L$ & 50 & 100 & EPA Method 8270 \\
\hline 2-sec-Butyl-4,6-dinitronhenol & $\mu \mathrm{g} / \mathrm{L}$ & 3.5 & 7 & Final PDWS (EPA, 1992b) \\
\hline Butylbenzyl phthalate & & No flag & No flag & Set by EPD/EMS \\
\hline Cadmium & $\mu g / L$ & 2.5 & 5 & Final PDWS (EPA, 1992a) \\
\hline Calcium & & No flag & No flag & Set by EPD/EMS \\
\hline Carbon disulfide & $\mu g / L$ & 5 & 10 & EPA Method 8240 \\
\hline Carbon tetrachloride & $\mu g / L$ & 2.5 & 5 & Final PDWS (EPA, 1992a) \\
\hline Carbon-14 & $\mathrm{pCi} / \mathrm{L}$ & $1 E+03$ & $2 E+O 3$ & Final PDWS (EPA, 1977) \\
\hline Carbonate & & No flag & No flag & Set by EPD/EMS \\
\hline Cerium-141 & $\mathrm{pCi} / \mathrm{L}$ & $1.5 \mathrm{E}+02$ & $3 E+02$ & Final PDWS (EPA, 1977) \\
\hline Cerium-144 & $\mathrm{pCi} / \mathrm{L}$ & $1.31 E+02$ & $2.61 E+02$ & Proposed PDWS (EPA, 1991) \\
\hline Cesium-134 ${ }^{b}$ & $\rho \mathrm{Ci} / \mathrm{L}$ & $4.07 E+01$ & $8.13 E+01$ & Proposed PDWS (EPA, 1991) \\
\hline Cesium-137 & $\rho \mathrm{Ci} / \mathrm{L}$ & $1 E+02$ & $2 E+02$ & Final PDWS (EPA, 1977) \\
\hline Chlordane & $\mu \mathrm{g} / \mathrm{L}$ & 1 & 2 & Final PDWS (EPA, 1992a) \\
\hline Chloride & $\mu \mathrm{g} / \mathrm{L}$ & 125,000 & 250,000 & SDWS (EPA, 1992c) \\
\hline 4-Chloroaniline & $\mu \mathrm{g} / \mathrm{L}$ & 50 & 100 & EPA Method 8270 \\
\hline Chlorobenzene & $\mu g / L$ & 50 & 100 & Final PDWS (EPA, 1992a) \\
\hline Chlorobenzilate & $\mu \mathrm{g} / \mathrm{L}$ & 50 & 100 & EPA Method 8270 \\
\hline Chloroethane & $\mu \mathrm{g} / \mathrm{L}$ & 5 & 10 & EPA Method 8240 \\
\hline Chloroethene (Vinyl chloride) & $\mu \mathrm{g} / \mathrm{L}$ & 1 & 2 & Final PDWS (EPA, 1992a) \\
\hline Chloroethyl vinyl ether & $\mu \mathrm{g} / \mathrm{L}$ & 5 & 10 & EPA Method 8240 \\
\hline 2-Chloroethyl vinyl ether & $\mu \mathrm{g} / \mathrm{L}$ & 5 & 10 & EPA Method 8240 \\
\hline Chloroform & $\mu \mathrm{g} / \mathrm{L}$ & 50 & 100 & Final PDWS (EPA, 1992a) \\
\hline 4-Chloro-m-cresol & $\mu \mathrm{g} / \mathrm{L}$ & 50 & 100 & EPA Method 8270 \\
\hline Chloromethane (Methyl chloride) & $\mu \mathrm{g} / \mathrm{L}$ & 5 & 10 & EPA Method 8240 \\
\hline 2-Chloronaphthalene & $\mu \mathrm{g} / \mathrm{L}$ & 50 & 100 & EPA Method 8240 \\
\hline 2-Chlorophenol & $\mu \mathrm{g} / \mathrm{L}$ & 50 & 100 & EPA Method 8270 \\
\hline 4-Chlorophenyl phenyl ether & $\mu \mathrm{g} / \mathrm{L}$ & 50 & 100 & EPA Method 8270 \\
\hline
\end{tabular}


WSRC-TR-93-277

\begin{tabular}{|c|c|c|c|c|}
\hline Analyte & Unit & Flag 1 & Flag 2 & Source $^{a}$ \\
\hline Chloroprene & $\mu \mathrm{g} / \mathrm{L}$ & 1,000 & 2,000 & EPA Method 8240 \\
\hline Chromium & $\mu \mathrm{g} / \mathrm{L}$ & 50 & 100 & Final PDWS (EPA, 1992a) \\
\hline Chromium -51 & $\mathrm{pCi} / \mathrm{L}$ & $3 E+03$ & $6 E+03$ & Final PDWS (EPA, 1977) \\
\hline Chrysene & $\mu g / L$ & 0.1 & 0.2 & Proposed PDWS (EPA, 1990) \\
\hline Cobalt & $\mu \mathrm{g} / \mathrm{L}$ & 20 & 40 & EPA Method 6010 \\
\hline Cobalt-57 & $\mathrm{pCi} / \mathrm{L}$ & $5 E+02$ & $1 E+03$ & Final PDWS (EPA, 1977) \\
\hline Cobalt -58 & $\mathrm{pCi} / \mathrm{L}$ & $4.5 E+03$ & $9 E+03$ & Final PDWS (EPA, 1977) \\
\hline Cobalt -60 & $\mathrm{pCi} / \mathrm{L}$ & $5 E+01$ & $1 E+02$ & Final PDWS (EPA, 1977) \\
\hline Color & & No flag & No flag & Set by EPD/EMS \\
\hline Copper & $\mu \mathrm{g} / \mathrm{L}$ & 650 & 1,300 & Final PDWS (EPA, 1992a) \\
\hline Corrosivity & & No flag & No flag & Set by EPD/EMS \\
\hline m-Cresol (3-Methylphenol) & $\mu g / L$ & 50 & 100 & EPA Method 8270 \\
\hline o-Cresol (2-Methylphenol) & $\mu g / L$ & 50 & 100 & EPA Method 8270 \\
\hline p-Cresol (4-Methylphenol) & $\mu g / L$ & 50 & 100 & EPA Method 8270 \\
\hline Curium-242 & $\mathrm{pCi} / \mathrm{L}$ & $6.65 E+01$ & $1.33 E+02$ & Proposed PDWS (EPA, 1991) \\
\hline Curium-243 & $\mathrm{pCi} / \mathrm{L}$ & $4.15 E+00$ & $8.3 E+00$ & Proposed PDWS (EPA, 1991) \\
\hline Curium-243/244 $\mathrm{c}$ & $\mathrm{pCi} / \mathrm{L}$ & $4.15 E+00$ & $8.3 E+00$ & Proposed PDWS (EPA, 1991) \\
\hline Curium-244 & $\mathrm{pCi} / \mathrm{L}$ & $4.92 E+00$ & $9.84 E+00$ & Proposed PDWS (EPA, 1991) \\
\hline Curium-245/246 ${ }^{c}$ & $\mathrm{pCi} / \mathrm{L}$ & $3.12 E+00$ & $6.23 E+00$ & Proposed PDWS (EPA, 1991) \\
\hline Curium-246 & $\mathrm{pCi} / \mathrm{L}$ & $3.14 E+00$ & $6.27 E+00$ & Proposed PDWS (EPA, 1991) \\
\hline Cyanide & $\mu g / L$ & 100 & 200 & Final PDWS (EPA, 1992b) \\
\hline$p, p^{\prime}-D D D$ & $\mu \mathrm{g} / \mathrm{L}$ & 0.5 & 1 & EPA Method 8080 \\
\hline$p, p^{\prime}-D D E$ & $\mu g / L$ & 0.5 & 1 & EPA Method 8080 \\
\hline$p, p^{\prime}-D D T$ & $\mu \mathrm{g} / \mathrm{L}$ & 0.5 & 1 & EPA Method 8080 \\
\hline Di-n-butyl phthalate & & No flag & No flag & Set by EPD/EMS \\
\hline Di-n-octyl phthalate & & No flag & No flag & Set by EPD/EMS \\
\hline Diallate & $\mu \mathrm{g} / \mathrm{L}$ & 50 & 100 & EPA Method 8270 \\
\hline Dibenzla,hlanthracene & $\mu \mathrm{g} / \mathrm{L}$ & 0.15 & 0.3 & Proposed PDWS (EPA, 1990) \\
\hline Dibenzofuran & $\mu \mathrm{g} / \mathrm{L}$ & 50 & 100 & EPA Method 8270 \\
\hline Dibromochloromethane & $\mu \mathrm{g} / \mathrm{L}$ & 50 & 100 & Final PDWS (EPA, 1992a) \\
\hline $\begin{array}{l}\text { 1,2-Dibromo-3-chloropropane } \\
\text { 1,2-Dibromoethane }\end{array}$ & $\mu \mathrm{g} / \mathrm{L}$ & 0.1 & 0.2 & Final PDWS (EPA, 1992a) \\
\hline (Ethylene dibromide) & $\mu \mathrm{g} / \mathrm{L}$ & 0.025 & 0.05 & Final PDWS (EPA, 1992a) \\
\hline Dibromomethane & & & & \\
\hline (Methylene bromide) & $\mu g / L$ & 5 & 10 & EPA Method 8240 \\
\hline 1,2-Dichlorobenzene & $\mu \mathrm{g} / \mathrm{L}$ & 300 & 600 & Final PDWS (EPA, 1992a) \\
\hline 1,3-Dichlorobenzene & $\mu \mathrm{g} / \mathrm{L}$ & 50 & 100 & EPA Method 8270 \\
\hline 1,4-Dichlorobenzene & $\mu \mathrm{g} / \mathrm{L}$ & 37.5 & 75 & Final PDWS (EPA, 1992a) \\
\hline 3,3'-Dichlorobenzidine & $\mu \mathrm{g} / \mathrm{L}$ & 50 & 100 & EPA Method 8270 \\
\hline trans-1,4-Dichloro-2-butene & $\mu \mathrm{g} / \mathrm{L}$ & 150 & 300 & EPA Method 8240 \\
\hline Dichlorodifluoromethane & $\mu g / L$ & 5 & 10 & EPA Method 8240 \\
\hline 1,1-Dichloroethane & $\mu g / L$ & 5 & 10 & EPA Method 8240 \\
\hline 1,2-Dichloroethane & $\mu g / L$ & 2.5 & 5 & Final PDWS (EPA, 1992a) \\
\hline 1,1-Dichloroethene & $\mu \mathrm{g} / \mathrm{L}$ & 3.5 & 7 & Final PDWS (EPA, 1992a) \\
\hline 1,2-Dichloroethene & $\mu \mathrm{g} / \mathrm{L}$ & 25 & 50 & Final PDWS (EPA, 1992b) \\
\hline cis-1,2-Dichloroethene & $\mu \mathrm{g} / \mathrm{L}$ & 35 & 70 & Final PDWS (EPA, 1992a) \\
\hline trans-1,2-Dichloroethene & $\mu \mathrm{g} / \mathrm{L}$ & 50 & 100 & Final PDWS (EPA, 1992a) \\
\hline Dichloromethane & & & & \\
\hline (Methylene chloride) & $\mu \mathrm{g} / \mathrm{L}$ & 2.5 & 5 & Final PDWS (EPA, 1992b) \\
\hline 2,4-Dichlorophenol & $\mu \mathrm{g} / \mathrm{L}$ & 50 & 100 & EPA Method 8270 \\
\hline 2,6-Dichlorophenol & $\mu \mathrm{g} / \mathrm{L}$ & 50 & 100 & EPA Method 8270 \\
\hline 2,4-Dichlorophenoxyacetic acid & $\mu \mathrm{g} / \mathrm{L}$ & 35 & 70 & Final PDWS (EPA, 1992a) \\
\hline 1,2-Dichloropropane & $\mu \mathrm{g} / \mathrm{L}$ & 2.5 & 5 & Final PDWS (EPA, 1992a) \\
\hline cis-1,3-Dichloropropene & $\mu g / L$ & 5 & 10 & EPA Method 8240 \\
\hline trans-1,3-Dichloropropene & $\mu g / L$ & 5 & 10 & EPA Method 8240 \\
\hline
\end{tabular}


WSRC-TR-93-277

\begin{tabular}{|c|c|c|c|c|}
\hline Analyte & $\underline{\text { Unit }}$ & Flag 1 & Flag 2 & Source $^{a}$ \\
\hline Dieldrin & $\mu \mathrm{g} / \mathrm{L}$ & 2.5 & 5 & EPA Method 8080 \\
\hline Diethyl phthalate & & No flag & No flag & Set by EPD/EMS \\
\hline Dimethoate & $\mu g / L$ & 50 & 100 & EPA Method 8270 \\
\hline p-Dimethylaminoazobenzene & $\mu g / L$ & $5 C$ & 100 & EPA Method 8270 \\
\hline p-(Dimethylaminolethylbenzene & $\mu \mathrm{g} / \mathrm{L}$ & 50 & 100 & EPA Method 8270 \\
\hline 7,12-Dimethylbenzla]anthracene & $\mu \mathrm{g} / \mathrm{L}$ & 50 & 100 & EFA Method 8270 \\
\hline 3,3'-Dimethylbenzidine & $\mu g / L$ & 50 & 100 & EPA Method 8270 \\
\hline a,a-Dimethylphenethylamine & $\mu g / L$ & 50 & 100 & EPA Method 8270 \\
\hline 2,4-Dimethyl phenol & $\mu g / L$ & 50 & 100 & EPA Method 8270 \\
\hline Dimethyl phthalate & & No flag & No flag & Set by EPD/EMS \\
\hline 1,3-Dinitrobenzene & $\mu g / L$ & 50 & 100 & EPA Method 8270 \\
\hline 2,4-Dinitrophenol & $\mu g / L$ & 250 & 500 & EPA Method 8270 \\
\hline 2,4-Dinitrotoluene & $\mu \mathrm{g} / \mathrm{L}$ & 50 & 100 & EPA Method 8270 \\
\hline 2,6-Dinitrotoluene & $\mu g / L$ & 50 & 100 & EPA Method 8270 \\
\hline 1.4-Dioxane & $\mu \mathrm{g} / \mathrm{L}$ & 50 & 100 & EPA Method 8270 \\
\hline Diphenylamine & $\mu g / L$ & 50 & 100 & EPA Method 8270 \\
\hline 1,2-Diphenylhydrazine & $\mu \mathrm{g} / \mathrm{L}$ & 50 & 100 & EPA Method 8270 \\
\hline Dissolved organic carbon & $\mu g / L$ & 5,000 & 10,000 & EPA Method 9060 \\
\hline Disulfoton & $\mu \mathrm{g} / \mathrm{L}$ & 50 & 100 & EPA Method 8270 \\
\hline Eh & & No flag & No flag & Set by EPD/EMS \\
\hline alpha-Endosulfan & $\mu \mathrm{g} / \mathrm{L}$ & 50 & 100 & EPA Method 8270 \\
\hline beta-Endosulfan & $\mu \mathrm{g} / \mathrm{L}$ & 50 & 100 & EPA Method 8270 \\
\hline Endosulfan I & $\mu \mathrm{g} / \mathrm{L}$ & 0.5 & 1 & EPA Method 8080 \\
\hline Endosulfan $\|$ & $\mu g / L$ & 0.5 & 1 & EPA Method 8080 \\
\hline Endosulfan sulfate & $\mu g / L$ & 0.5 & 1 & EPA Method 8080 \\
\hline Endrin & $\mu g / L$ & 1 & 2 & Final PDWS (EPA, 1992b) \\
\hline Endrin aldehyde & $\mu \mathrm{g} / \mathrm{L}$ & 0.5 & 1 & EPA Method 8080 \\
\hline Endrin ketorie & & No flag & No flag & Set by EPD/EMS \\
\hline Ethylbenzene & $\mu g / L$ & 350 & 700 & Final PDWS (EPA, 1992a) \\
\hline Ethyl methacrylate & $\mu g / L$ & 50 & 100 & EPA Method 8270 \\
\hline Ethyl methanesulforiaie & $\mu \mathrm{g} / \mathrm{L}$ & 50 & 100 & EPA Method 8270 \\
\hline Europium-152 & $\mathrm{pCi} / \mathrm{L}$ & $3 E+01$ & $6 E+01$ & Final PDWS (EPA, 1977) \\
\hline Europium-154 & $\mathrm{pCi} / \mathrm{L}$ & $1 E+02$ & $2 E+02$ & Final PDWS (EPA, 1977) \\
\hline Europium-155 & $\mathrm{pCi} / \mathrm{L}$ & $3 E+02$ & $6 E+02$ & Final PDWS (EPA, 1977) \\
\hline Famphur & $\mu \mathrm{g} / \mathrm{L}$ & 50 & 100 & EPA Miethod 8270 \\
\hline Fluoranthene & $\mu g / L$ & 50 & 100 & EPA Method 8270 \\
\hline Fluorene & $\mu g / L$ & 50 & 100 & EPA Method 8270 \\
\hline Fluoride & $\mu \mathrm{g} / \mathrm{L}$ & 2,000 & 4,000 & Fina! PDWS (EPA, 1992a) \\
\hline Gross alpha & $\mathrm{pCi} / \mathrm{L}$ & $7.5 E+00$ & $1.5 E+01$ & Final PDWS (EPA, 1992a) \\
\hline Heptachlor & $\mu g / L$ & 0.2 & 0.4 & Final PDWS (EPA, 1992a) \\
\hline Heptachlor epoxide & $\mu g / L$ & 0.1 & 0.2 & Final PDWS (EPA, 1992a) \\
\hline Heptachlorodibenzo-p-dioxin & & & & \\
\hline isomers & $\mu \mathrm{g} / \mathrm{L}$ & 0.00325 & 0.0065 & EPA Method 8280 \\
\hline $1,2,3,4,6,7,8-\mathrm{HPCDD}$ & $\mu \mathrm{g} / \mathrm{L}$ & 0.00325 & 0.0065 & EPA Method 8280 \\
\hline $\begin{array}{l}\text { Heptachlorodibenzo-p-furan } \\
\text { isomers }\end{array}$ & & & 0.0045 & EPA Method 8280 \\
\hline $\begin{array}{l}\text { isomers } \\
1,2,3,4,6,7,8 \text {-HPCDF }\end{array}$ & $\mu g / L$ & $\begin{array}{l}0.00225 \\
0.00225\end{array}$ & 0.0045 & EPA Method 8280 \\
\hline Hexachlorobenzene & $\begin{array}{l}\mu \mathrm{g} / \mathrm{L} \\
\mu \mathrm{g} / \mathrm{L}\end{array}$ & 0.5 & 1 & Final PDWS (EPA, 1992b) \\
\hline Hexachlorobutadiene & $\mu \mathrm{g} / \mathrm{L}$ & 50 & 100 & EPA Method 8270 \\
\hline Hexachlorocyclopentadiene & $\mu g / L$ & 25 & 50 & Final PDWS (EPA, 1992t) \\
\hline Hexachlorodibenzo-p-dioxin isomers & $\mu \mathrm{g} / \mathrm{L}$ & 0.00225 & 0.0045 & EPA Method 8280 \\
\hline $1,2,3,4,7,8-\mathrm{H} \times C D D$ & $\mu \mathrm{g} / \mathrm{L}$ & 0.00225 & 0.0045 & EPA Method 8280 \\
\hline Hexachlorodibenzo-p-furan isomers & $\mu \mathrm{g} / \mathrm{L}$ & 0.002 & 0.004 & EPA Method 8280 \\
\hline $1,2,3,4,7,8-\mathrm{HXCDF}$ & $\mu g / L$ & 0.002 & 0.004 & EPA Method 8280 \\
\hline Hexachloroethane & $\mu \mathrm{g} / \mathrm{L}$ & 50 & 100 & EPA Method 8270 \\
\hline
\end{tabular}




\begin{tabular}{|c|c|c|c|c|}
\hline Analyte & Unit & Flag 1 & Flag 2 & $\underline{\text { Source }}^{a}$ \\
\hline Hexachlorophene & $\mu \mathrm{g} / \mathrm{L}$ & 250 & 500 & EPA Method 8270 \\
\hline H乞xachloropropene & $\mu \mathrm{g} / \mathrm{L}$ & 50 & 100 & EPA Method 8270 \\
\hline 2-Hexanone & $\mu g / L$ & 50 & 100 & EPA Method 8240 \\
\hline Indeno[1,2,3-c,o]pyrene & $\mu \mathrm{g} / \mathrm{l}$ & 50 & 100 & EPA Method 8270 \\
\hline lodine & $\mu g / l$ & 250 & 500 & APHA Method 415A \\
\hline lodine-129 & $\mathrm{pCi} / \mathrm{L}$ & $5 E-01$ & $1 E+00$ & Final PDWS :PA, 1977) \\
\hline lodine-131 & $\mathrm{pCi}, \mathrm{L}$ & $1.5 E+00$ & $3 E+00$ & Final Pr, rvs (EPA, 1977) \\
\hline lodomethane (Methyl iodide) & $\mu \mathrm{g} / \mathrm{l}$ & 75 & 150 & EPA Method 8240 \\
\hline Iron & $\mu g / L$ & 150 & 300 & SDWS (EPA, 1992c) \\
\hline Iron-55 & $\mathrm{pCi} / \mathrm{L}$ & $1 E+03$ & $2 E+03$ & Final PDWS (EPA, 1977) \\
\hline Iron-59 & $\mathrm{pCi} / \mathrm{L}$ & $1 E+02$ & $2 E+02$ & Final PDWS IEPA, 1977: \\
\hline |sobuty| alcohol & $\mu g / L$ & 500 & 1,000 & EPA Method 8240 \\
\hline Isodrin & $\mu \mathrm{g} / \mathrm{L}$ & 50 & 100 & EPA Method 8270 \\
\hline Isophorone & $\mu g / L$ & 50 & 100 & EPA Method 8270 \\
\hline Isosafrole & $\mu \mathrm{g} / \mathrm{L}$ & 50 & 100 & EPA Method 8270 \\
\hline Kepone & $\mu \mathrm{g} / \mathrm{L}$ & 50 & 100 & EPA Method 8270 \\
\hline Lanthanum-140 & $\mathrm{pCi} / \mathrm{L}$ & $3 E+01$ & $6 E+01$ & Final PDWS (EPA, 1977) \\
\hline Lead & $\mu \mathrm{g} / \mathrm{L}$ & 7.5 & 15 & Final PDWS (EPA, 1992a) \\
\hline Lindane & $\mu \mathrm{g} / \mathrm{L}$ & 0.1 & 0.2 & Final PDWS (EPA, 1992a) \\
\hline Lithium & $\mu \mathrm{g} / \mathrm{L}$ & 25 & 50 & EPA Method 6010 \\
\hline Magnesium & & No flag & No flag & Set by EPD/EMS \\
\hline Manganese & $\mu \mathrm{g} / \mathrm{L}$ & 25 & 50 & SDWS (EPA, 1992c) \\
\hline Manganese-54 & $\mathrm{pCi} / \mathrm{L}$ & $1.5 E+02$ & $3 E+02$ & Final PDWS (EPA, 1977) \\
\hline Mercury & $\mu \mathrm{g} / \mathrm{L}$ & 1 & 2 & Final PDWS (EPA, 1992a) \\
\hline Methacrylonitrile & $\mu \mathrm{g} / \mathrm{L}$ & 250 & 500 & EPA Method 8240 \\
\hline Methapyrilene & $\mu \mathrm{g} / \mathrm{L}$ & 50 & 100 & EPA Method 8270 \\
\hline Methoxychior & $\mu \mathrm{g} / \mathrm{L}$ & 20 & 40 & Final PDWS (EPA, 1992a) \\
\hline 3-Methylcholanthrene & $\mu \mathrm{g} / \mathrm{L}$ & 50 & 100 & EPA Method 8270 \\
\hline 2-Methyl-4,6-dinitrophenol & $\mu \mathrm{g} / \mathrm{L}$ & 250 & 500 & EPA Method 8270 \\
\hline Methyl ethyl ketone & & No flag & No flag & Set by EPD/EMS \\
\hline Methyl isobutyl ketone & & No flag & No flag & Set by EPD/EMS \\
\hline Methyl methacrylate & $\mu \mathrm{g} / \mathrm{L}$ & 50 & 100 & EPA Method 8270 \\
\hline Methyl methanesulfonate & $\mu g / L$ & 50 & 100 & EPA Method 8270 \\
\hline ?-Methylnaphthalene & $\mu \mathrm{g} / \mathrm{L}$ & 50 & 100 & EPA Method 8270 \\
\hline Molybdenum & $\mu g / L$ & 250 & 500 & EPA Method 6010 \\
\hline Naphthalene & $\mu g / L$ & 50 & 100 & EPA Method 8270 \\
\hline 1,4-Naphthoquinone & $\mu \mathrm{g} / \mathrm{L}$ & 50 & 100 & EPA Method 8270 \\
\hline 1-Naphthylamine & $\mu \mathrm{g} / \mathrm{L}$ & 50 & 100 & EPA Method 8270 \\
\hline 2-Naphthylamine & $\mu \mathrm{g} / \mathrm{L}$ & 50 & 100 & EPA Method 8270 \\
\hline Neptunium-237 & $\mathrm{pCi} / \mathrm{L}$ & $3.53 E+00$ & $7.06 E+00$ & Proposed PDWS (EPA, 1991) \\
\hline Nickel & $\mu \mathrm{g} / \mathrm{L}$ & 50 & 100 & Final PDWS (EPA, 1992b) \\
\hline Nickel-59 & $\mathrm{pCi} / \mathrm{L}$ & $1.5 E+02$ & $3 E+02$ & Final PDWS (EPA, 1977) \\
\hline Nickel-63 & $\mathrm{pCi} / \mathrm{L}$ & $2.5 E+01$ & $5 E+01$ & Final PDWS (EPA, 1977) \\
\hline Niobium-95 & $\mathrm{pCi} / \mathrm{L}$ & $1.5 E+02$ & $3 . E+02$ & Final PDWS (EPA, 1977) \\
\hline Nitrate as nitrogen & $\mu \mathrm{g} / \mathrm{L}$ & 5,000 & 10,000 & Final PDWS (EPA, 1992a) \\
\hline Nitrate-nitrite as nitrogen & $\mu g / L$. & 5,000 & 10,000 & Final PDWS (EPA, 1992a) \\
\hline Nitrite as nitrogen & $\mu \mathrm{g} / \mathrm{L}$ & 500 & 1,000 & Final PDWS (EPA, 1992a) \\
\hline 2-Nitroaniline & $\mu \mathrm{g} / \mathrm{L}$ & 50 & 100 & EPA Method 8270 \\
\hline 3-Nitroaniline & $\mu g / L$ & 50 & 100 & EPA Method 8270 \\
\hline 4-Nitroaniline & $\mu \mathrm{g} / \mathrm{L}$ & 50 & 100 & EPA Method 8270 \\
\hline Nitrobenzene & $\mu \mathrm{g} / \mathrm{L}$ & 50 & 100 & EPA Method 8270 \\
\hline Nitrogen by Kjeldahl method & $\mu \mathrm{g} / \mathrm{L}$ & 500 & 1,000 & EPA Method 351.2 \\
\hline 2-Nitrophenol & $\mu g / L$ & 50 & 100 & EPA Method 8270 \\
\hline 4-Nitrophenol & $\mu \mathrm{g} / \mathrm{L}$ & 50 & 100 & EPA Method 8270 \\
\hline 4-Nitroquinoline-1-oxide & $\mu \mathrm{g} / \mathrm{L}$ & 50 & 100 & EPA Method 8270 \\
\hline
\end{tabular}


WSRC-TR-93-277

\begin{tabular}{|c|c|c|c|c|}
\hline Analyte & $\underline{\text { Unit }}$ & Flag 1 & Flag 2 & $\underline{\text { Source }}^{a}$ \\
\hline N-Nitrosodi-n-butylamine & $\mu g / L$ & 50 & 100 & EPA Method 8270 \\
\hline N-Nitrosodiethylamine & $\mu g / L$ & 50 & 100 & EPA Method 8270 \\
\hline N-Nitrosodimethylamine & $\mu \mathrm{g} / \mathrm{L}$ & 50 & 100 & EPA Method 8270 \\
\hline N-Nitrosodiphenylamine & $\mu \mathrm{g} / \mathrm{L}$ & 50 & 100 & EPA Method 8270 \\
\hline N-Nitrosodipropylamine & $\mu \mathrm{g} / \mathrm{L}$ & 50 & 100 & EPA Method 8270 \\
\hline $\mathrm{N}$-Nitrosomethylethylamine & $\mu \mathrm{g} / \mathrm{L}$ & 50 & 100 & EPA Method 8270 \\
\hline N-Nitrosomorpholine & $\mu \mathrm{g} / \mathrm{L}$ & 50 & 100 & EPA Method 8270 \\
\hline N-Nitrosopiperidine & $\mu \mathrm{g} / \mathrm{L}$ & 50 & 100 & EPA Method 8270 \\
\hline N-Nitrosopyrrolidine & $\mu \mathrm{g} / \mathrm{L}$ & 50 & 100 & EPA Method 8270 \\
\hline 5-Nitro-0-toluidine & $\mu \mathrm{g} / \mathrm{L}$ & 50 & 100 & EPA Method 8270 \\
\hline Nonvolatile beta & $\mathrm{pCi} / \mathrm{L}$ & $2.5 E+01$ & $5 E+01$ & Proposed PDWS (EPA, 1986) \\
\hline Octachlorodibenzo-p-dioxin isomers & $\mu \mathrm{g} / \mathrm{L}$ & 0.005 & 0.01 & EPA Method 8280 \\
\hline Octachlorodibenzo-p-furan isomers & $\mu g / L$ & 0.005 & 0.01 & EPA Method 8280 \\
\hline Odor & & No flag & No flag & Set by EPD/EMS \\
\hline Oil \& Grease & $\mu g / L$ & 5,000 & 10,000 & EPA Method 413.1 \\
\hline Parathion & $\mu g / L$ & 0.25 & 0.5 & EPA Method 8080 \\
\hline Parathion methyl & $\mu \mathrm{g} / \mathrm{L}$ & 0.25 & 0.5 & EPA Method 8080 \\
\hline PCB 1016 & $\mu \mathrm{g} / \mathrm{L}$ & 0.25 & 0.5 & Final PDWS (EPA, 1992a) \\
\hline PCB 1221 & $\mu \mathrm{g} / \mathrm{L}$ & 0.25 & 0.5 & Final PDWS (EPA, 1992a) \\
\hline PCB 1232 & $\mu \mathrm{g} / \mathrm{L}$ & 0.25 & 0.5 & Final PDWS (EPA, 1992a) \\
\hline PCB 1242 & $\mu \mathrm{g} / \mathrm{L}$ & 0.25 & 0.5 & Final PDWS (EPA, 1992a') \\
\hline PCB 1248 & $\mu \mathrm{g} / \mathrm{L}$ & 0.25 & 0.5 & Final PDWS (EPA, 1992a) \\
\hline PCB 1254 & $\mu \mathrm{g} / \mathrm{L}$ & 0.25 & 0.5 & Final PDWS (EPA, 1992a) \\
\hline PCB 1260 & $\mu \mathrm{g} / \mathrm{L}$ & 0.25 & 0.5 & Final PDW/S (EPA, 1992a) \\
\hline PCB 1262 & $\mu \mathrm{g} / \mathrm{L}$ & 0.25 & 0.5 & Final PDWS (EPA, 1992a) \\
\hline Pentachlorobenzene & $\mu \mathrm{g} / \mathrm{L}$ & 50 & 100 & EPA Method 8270 \\
\hline Pentachlorodibenzo-p-dioxin isomers & $\mu \mathrm{g} / \mathrm{L}$ & 0.00275 & 0.0055 & EPA Method 8280 \\
\hline $1,2,3,7,8-P C D D$ & $\mu \mathrm{g} / \mathrm{L}$ & 0.00275 & 0.0055 & EPA Method 8280 \\
\hline Pentachlorodibenzo-p-furan isomers & $\mu \mathrm{g} / \mathrm{L}$ & 0.00275 & 0.0055 & EPA Method 8280 \\
\hline $1,2,3,7,8-P C D F$ & $\mu \mathrm{g} / \mathrm{L}$ & 0.00275 & 0.0055 & EPA Method 8280 \\
\hline Pentachloroethane & $\mu \mathrm{g} / \mathrm{L}$ & 50 & 100 & EPA Method 8270 \\
\hline Pentachloronitrobenzene & $\mu \mathrm{g} / \mathrm{L}$ & 50 & 100 & EPA Method 8270 \\
\hline Pentachlorophenol & $\mu \mathrm{g} / \mathrm{L}$ & 0.5 & 1 & Final PDWS (EPA, 1992a) \\
\hline $\mathrm{pH}$ & $\mathrm{pH}$ & 8 & 10 & Set by EPD/EMS \\
\hline $\mathrm{pH}$ & $\mathrm{pH}$ & 4 & 3 & Set by EPD/EMS \\
\hline Phenacetin & $\mu g / L$ & 50 & 100 & EPA Method 8270 \\
\hline Phenanthrene & $\mu g / L$ & 50 & 100 & EPA Method 8270 \\
\hline Phenol & $\mu \mathrm{g} / \mathrm{L}$ & 50 & 100 & EPA Method 8270 \\
\hline Phenols & $\mu \mathrm{g} / \mathrm{L}$ & 25 & 50 & EPA Method 420.1 \\
\hline p-Phenylenediamine & $\mu \mathrm{g} / \mathrm{L}$ & 50 & 100 & EPA Method 8270 \\
\hline Phorate & $\mu \mathrm{g} / \mathrm{L}$ & 0.5 & 1 & EPA Method 8080 \\
\hline 2-Picoline & $\mu \mathrm{g} / \mathrm{L}$ & 50 & 100 & EPA Method 8270 \\
\hline Plutonium-238 & $\mathrm{pCi} / \mathrm{L}$ & $3.51 E+00$ & $7.02 E+00$ & Proposed PDWS (EPA, 1991) \\
\hline Plutonium-239 & $\mathrm{pCi} / \mathrm{L}$ & $3.11 E+01$ & $6.21 E+01$ & Proposed PDWS (EPA, 1991) \\
\hline Plutonium-239/240 & $\mathrm{pCi} / \mathrm{L}$ & $3.11 E+01$ & $6.21 E+01$ & Proposed PDWS (EPA, 1991) \\
\hline Plutonium-240 & $\mathrm{pCi} / \mathrm{L}$ & $3.11 E+01$ & $6.22 E+01$ & Proposed PDWS (EPA, 1991 ) \\
\hline Plutonium-241 & $\mathrm{pCi} / \mathrm{L}$ & $3.13 E+01$ & $6.26 E+01$ & Proposed PDWS (EPA, 1991) \\
\hline Plutonium-242 & $\mathrm{pCi} / \mathrm{L}$ & $3.27 E+01$ & $6.54 E+01$ & Proposed PDWS (EPA, 1991 ) \\
\hline Potassium & & No flag & No flag & Set by EPD/EMS \\
\hline Potassium-40 & $\mathrm{pCi} / \mathrm{L}$ & $1.5 E+02$ & $3 E+02$ & Proposed PDWS (EPA, 1986) \\
\hline Pronamid & $\mu \mathrm{g} / \mathrm{L}$ & 50 & 100 & EPA Method 8270 \\
\hline Propionitrile & $\mu \mathrm{g} / \mathrm{L}$ & 1,000 & 2,000 & EPA Method 8240 \\
\hline Pyrene & $\mu \mathrm{g} / \mathrm{L}$ & 50 & 100 & EPA Method 8270 \\
\hline Pyridine & $\mu \mathrm{g} / \mathrm{L}$ & 50 & 100 & EPA Method 8270 \\
\hline Radium |alpha-emitting $\left.\right|^{d}$ & pCill & $1 E+1$ & $2 E+01$ & Proposed PDWS (EPA, 1991! \\
\hline
\end{tabular}




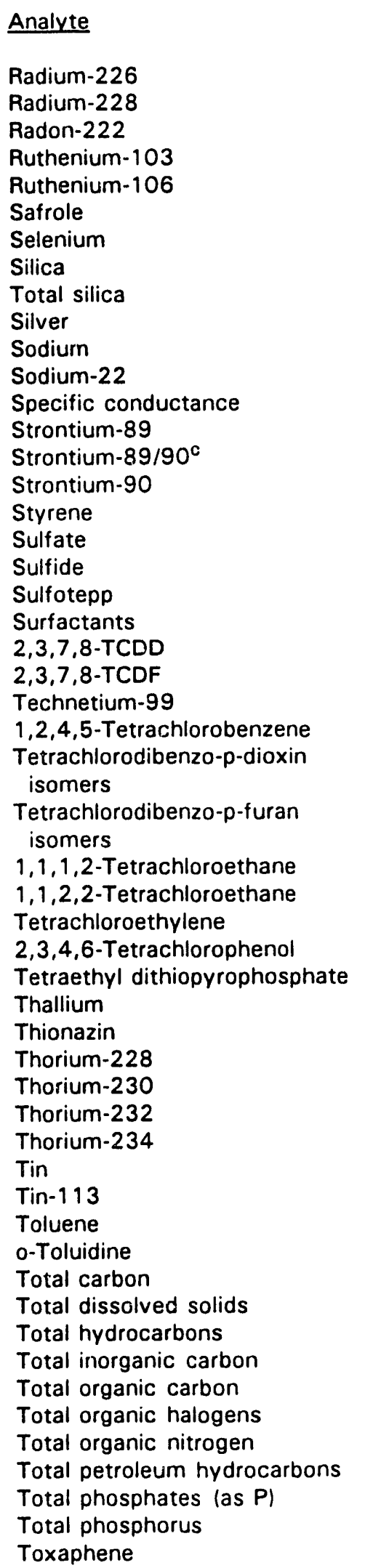

\begin{tabular}{|c|c|c|c|}
\hline Unit & Fiag 1 & Flag 2 & Source $^{a}$ \\
\hline $\mathrm{pCi} / \mathrm{L}$ & $1 E+01$ & $2 E+01$ & Proposed PDWS (EPA, 1991) \\
\hline $\mathrm{pCi} / \mathrm{L}$ & $1 E+01$ & $2 E+01$ & Proposed PDWS (EPA, 1991) \\
\hline $\mathrm{pCi} / \mathrm{L}$ & $1.5 E+02$ & $3 E+02$ & Proposed PDWS (EPA, 1991) \\
\hline $\mathrm{pCi} / \mathrm{L}$ & $1 E+02$ & $2 E+02$ & Final PDWS (EPA, 1977) \\
\hline $\mathrm{pCi} / \mathrm{L}$ & $1.5 E+01$ & $3 E+01$ & Final PDWS (EPA, 1977) \\
\hline$\mu \mathrm{g} / \mathrm{L}$ & 50 & 100 & EPA Method 8270 \\
\hline$\mu \mathrm{g} / \mathrm{L}$ & 25 & 50 & Final PDWS (EPA, 1992a) \\
\hline & No flag & No flag & Set by EPD/EMS \\
\hline$\mu g / L$ & 500 & 1,000 & EPA Method 6010 \\
\hline$\mu g / L$ & 50 & 100 & SDWS (EPA, 1992c) \\
\hline & No flag & No flag & Set by EPD/EMS \\
\hline $\mathrm{pCi} / \mathrm{L}$ & $2.33 E+02$ & $4.66 \mathrm{E}+02$ & Proposed PDWS (EPA, 1991) \\
\hline$\mu \mathrm{S} / \mathrm{cm}$ & 250 & 500 & Set by EPD/EMS \\
\hline $\mathrm{pCi} / \mathrm{L}$ & $1 E+01$ & $2 E+01$ & Final PDWS (EPA, 1977) \\
\hline $\mathrm{pCi} / \mathrm{L}$ & $4 E+00$ & $8 E+00$ & Final PDWS (EPA, 1992a) \\
\hline $\mathrm{pCi} / \mathrm{L}$ & $4 E+00$ & $8 E+00$ & Final PDWS (EPA, 1992a) \\
\hline$\mu g / L$ & 50 & 100 & Final PDWS (EPA, 1992a) \\
\hline$\mu \mathrm{g} / \mathrm{L}$ & 200,000 & 400,000 & Proposed PDWS (EPA, 1990 ) \\
\hline$\mu \mathrm{g} / \mathrm{L}$ & 5,000 & 10,000 & EPA Method 9030 \\
\hline$\mu \mathrm{g} / \mathrm{L}$ & 50 & 100 & EPA Method 8270 \\
\hline & No flag & No flag & Set by EPD/EMS \\
\hline$\mu \mathrm{g} / \mathrm{L}$ & 0.000015 & 0.00003 & Final PDWS (EPA, 1992b) \\
\hline$\mu \mathrm{g} / \mathrm{L}$ & 0.002 & 0.004 & EPA Method 8280 \\
\hline $\mathrm{pCi} / \mathrm{L}$ & $4.5 E+02$ & $9 E+02$ & Final PDWS (EPA, 1977) \\
\hline$\mu \mathrm{g} / \mathrm{L}$ & 50 & 100 & EPA Method 8270 \\
\hline$\mu \mathrm{g} / \mathrm{L}$ & 0.00225 & 0.0045 & EPA Method 8280 \\
\hline$\mu g / L$ & 0.002 & 0.004 & EPA Method 8280 \\
\hline$\mu g / L$ & 5 & 10 & EPA Method 8240 \\
\hline$\mu \mathrm{g} / \mathrm{L}$ & 5 & 10 & EPA Method 8240 \\
\hline$\mu g / L$ & 2.5 & 5 & Final PDWS (EPA, 1992a) \\
\hline$\mu \mathrm{g} / \mathrm{L}$ & 50 & 100 & EPA Method 8270 \\
\hline$\mu \mathrm{g} / \mathrm{L}$ & 50 & 100 & EPA Method 8270 \\
\hline$\mu \mathrm{g} / \mathrm{L}$ & 1 & 2 & Final PDWS (EPA, 1992b) \\
\hline$\mu g / L$ & 50 & 100 & EPA Method 8270 \\
\hline $\mathrm{pCi} / \mathrm{L}$ & $6.25 E+01$ & $1.25 E+02$ & Proposed PDWS (EPA, 1991) \\
\hline $\mathrm{pCi} / \mathrm{L}$ & $3.96 E+01$ & $7.92 E+01$ & Proposed PDWS (EPA, 1991) \\
\hline $\mathrm{pCi} / \mathrm{L}$ & $4.4 \mathrm{E}+01$ & $8.8 E+01$ & Proposed PDWS (EPA, 1991) \\
\hline $\mathrm{pCi} / \mathrm{L}$ & $2 E+02$ & $4.01 E+02$ & Proposed PDWS (EPA, 1991) \\
\hline$\mu g / L$ & 10 & 20 & EPA Method 282.2 \\
\hline $\mathrm{pCi} / \mathrm{L}$ & $1.5 E+02$ & $3 E+02$ & Final PDWS (EPA, 1977) \\
\hline$\mu \mathrm{g} / \mathrm{L}$ & 500 & 1,000 & Final PDWS (EPA, 1992a) \\
\hline$\mu \mathrm{g} / \mathrm{L}$ & 50 & 100 & EPA Method 8270 \\
\hline$\mu \mathrm{g} / \mathrm{L}$ & 5,000 & 10,000 & EPA Method 9060 \\
\hline & No flag & No flag & Set by EPD/EMS \\
\hline$\mu g / L$ & 5,000 & 10,000 & EPA Method 418.1 \\
\hline$\mu g / L$ & 5,000 & 10,000 & EPA Method 9060 \\
\hline$\mu g / L$ & 5,000 & 10,000 & EPA Method 9060 \\
\hline$\mu \mathrm{g} / \mathrm{L}$ & 25 & 50 & EPA Method 9020 \\
\hline$\mu g / L$ & 500 & 1,000 & APHA Method 420 \\
\hline$\mu \mathrm{g} / \mathrm{L}$ & 5,000 & 10,000 & EPA Method 418.1 \\
\hline & No flag & No flag & Set by EPD/EMS \\
\hline & No flag & No flag & Set by EPD/EMS \\
\hline$g / L$ & 1.5 & 3 & Final PDWS (EPA, 1992a) \\
\hline
\end{tabular}


Analyte

2,4,5-TP (Silvex)

Tributyl phosphate

1,2,4-Trichlorobenzene

1,1,1-Trichloroethane

1,1,2-Trichloroethane

Trichloroethylene

Trichlorofluoromethane

2,4,5-Trichlorophenol

2,4,6-Trichlorophenol

$2,4,5$-Trichlorophenoxyacetic acid

1,2,3-Trichloropropane

$0,0,0$-Triethyl phosphorothioate

1,3,5-Trinitrobenzene

Tritium

Turbidity

Uranium

Uranium alpha activity

Uranium-233/234 ${ }^{c}$

Uranium-234

Uranium-235

Uranium-238

Vanadium

Vinyl acetate

Xylenes

Zinc

Zinc-65

Zirconium-95

Zirconium/Niobium-95c

\begin{tabular}{|c|c|c|}
\hline Unit & Flag 1 & Flag 2 \\
\hline$\mu \mathrm{g} / \mathrm{L}$ & 25 & 50 \\
\hline$\mu \mathrm{g} / \mathrm{L}$ & 50 & 100 \\
\hline$\mu \mathrm{g} / \mathrm{L}$ & 35 & 70 \\
\hline$\mu \mathrm{g} / \mathrm{L}$ & 100 & 200 \\
\hline$\mu \mathrm{g} / \mathrm{L}$ & 2.5 & 5 \\
\hline$\mu g / L$ & 2.5 & 5 \\
\hline$\mu \mathrm{g} / \mathrm{L}$ & 5 & 10 \\
\hline$\mu g / L$ & 50 & 100 \\
\hline$\mu \mathrm{g} / \mathrm{L}$ & 50 & 100 \\
\hline$\mu g / L$ & 2.5 & 5 \\
\hline$\mu g / L$ & 5 & 10 \\
\hline$\mu \mathrm{g} / \mathrm{L}$ & 50 & 100 \\
\hline$\mu \mathrm{g} / \mathrm{L}$ & 50 & 100 \\
\hline $\mathrm{pCi} / \mathrm{mL}$ & $1 E+01$ & $2 E+01$ \\
\hline & No flag & No flag \\
\hline$\mu \mathrm{g} / \mathrm{L}$ & 10 & 20 \\
\hline$p C i / L$ & $1.5 E+01$ & $3 E+01$ \\
\hline $\mathrm{pCi} / \mathrm{L}$ & $6.9 E+00$ & $1.38 E+01$ \\
\hline $\mathrm{pCi} / \mathrm{L}$ & $6.95 E+00$ & $1.39 E+01$ \\
\hline $\mathrm{pCi} / \mathrm{L}$ & $7.25 E+00$ & $1.45 E+01$ \\
\hline$p C i / L$ & $7.3 E+00$ & $1.46 E+01$ \\
\hline$\mu g / L$ & 40 & 80 \\
\hline$\mu \mathrm{g} / \mathrm{L}$ & 5 & 10 \\
\hline$\mu \mathrm{g} / \mathrm{L}$ & 5,000 & 10,000 \\
\hline$\mu \mathrm{g} / \mathrm{L}$ & 2,500 & 5,000 \\
\hline $\mathrm{pCi} / \mathrm{L}$ & $1.5 E+02$ & $3 E+02$ \\
\hline $\mathrm{pCi} / \mathrm{L}$ & $1 E+02$ & $2 E+02$ \\
\hline $\mathrm{pCi} / \mathrm{L}$ & $1 E+02$ & $2 E+02$ \\
\hline
\end{tabular}

$\underline{\text { Source }}^{\mathrm{a}}$

Final PDWS (EPA, 1992a)

EPA Method 8270

Final PDWS (EPA, 1992b)

Final PDWS (EPA, 1992a)

Final PDWS (EPA, 1992b)

Final PDWS (EPA, 1992a)

EPA Method 8240

EPA Method 8270

EPA Method 8270

EPA Method 8150

EPA Method 8240

EPA Method 8270

EPA Method 8270

Final PDWS (EPA, 1992a)

Set by EPD/EMS

Proposed PDWS (EPA, 1991)

Proposed PDWS (EPA, 1991)

Proposed PDWS (EPA, 1991)

Proposed PDWS (EPA, 1991)

Proposed PDWS (EPA, 1991)

Proposed PDWS (EPA, 1991)

EPA Method 6010

EPA Method 8240

Final PDWS (EPA, 1992a)

SDWS (EPA, 1992C)

Final PDWS (EPA, 1977)

Final PDWS (EPA, 1977)

Final PDWS (EPA, 1977)

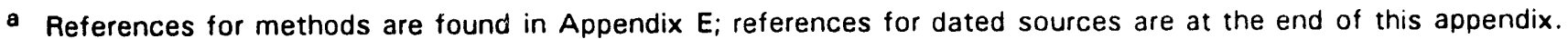

b EPD/EMS set this flagging criterion using the 1991 proposed PDWS because the final PDWS in 1977 may have been in error.

c When radionuclide analyses are combined, the lower PDWS of the two isotopes is used for flagging.

d The applied standard is for radium-226.

\section{References}

EPA (U.S. Environmental Protection Agency), 1977. National Interim Primary Drinking Water Regulations, EPA-570/9-76-003. Washington, DC.

EPA (U.S. Environmental Protection Agency), 1986. Water Pollution Control; National Primary Drinking Water Regulations, Radionuclides (Proposed). Federal Register, September 30, 1986, pp. 34836-34862. Washington, DC.

EPA (U.S. Environmental Protection Agency), 1990. National Primary and Secondary Drinking Water Regulations; Synthetic Organic Chemicals and Inorganic Chemicals (Proposed Rule). Federal Register, July 25, 1990. pp. 30369-30448. Washington, DC.

EPA (U.S. Environmental Protection Agency), 1991. National Primary Drinking Water Regulations; Radionuclides; Proposed Rule. Federal Register, July 18, 1991, pp. 33052-33127. Washington, DC. 
EPA (U.S. Environmental Protection Agencyl, 1992a. National Primary Drinking Water Regulations, Code of Federal Regulations, Section 40, Part 141, pp. 589-729. Washington, DC.

EPA (U.S. Environmental Protection Agency), 1992b. National Primary Drinking Water Regulations-Synthetic Organic Chemical and Inorganic Chemicals; National Primary Drinking Water Regulations Implementation. Federal Register, July 17, 1992, pp. 31776-31849. Washington, DC.

EPA (U.S. Environmental Protection Agency), 1992c. National Secondary Drinking Water Regulations, Code of Federal Regulations, Section 40, Part 143, pp. 772-776. Washington, DC. 
WSRC-TR-93-277

Appendix C - Figures 


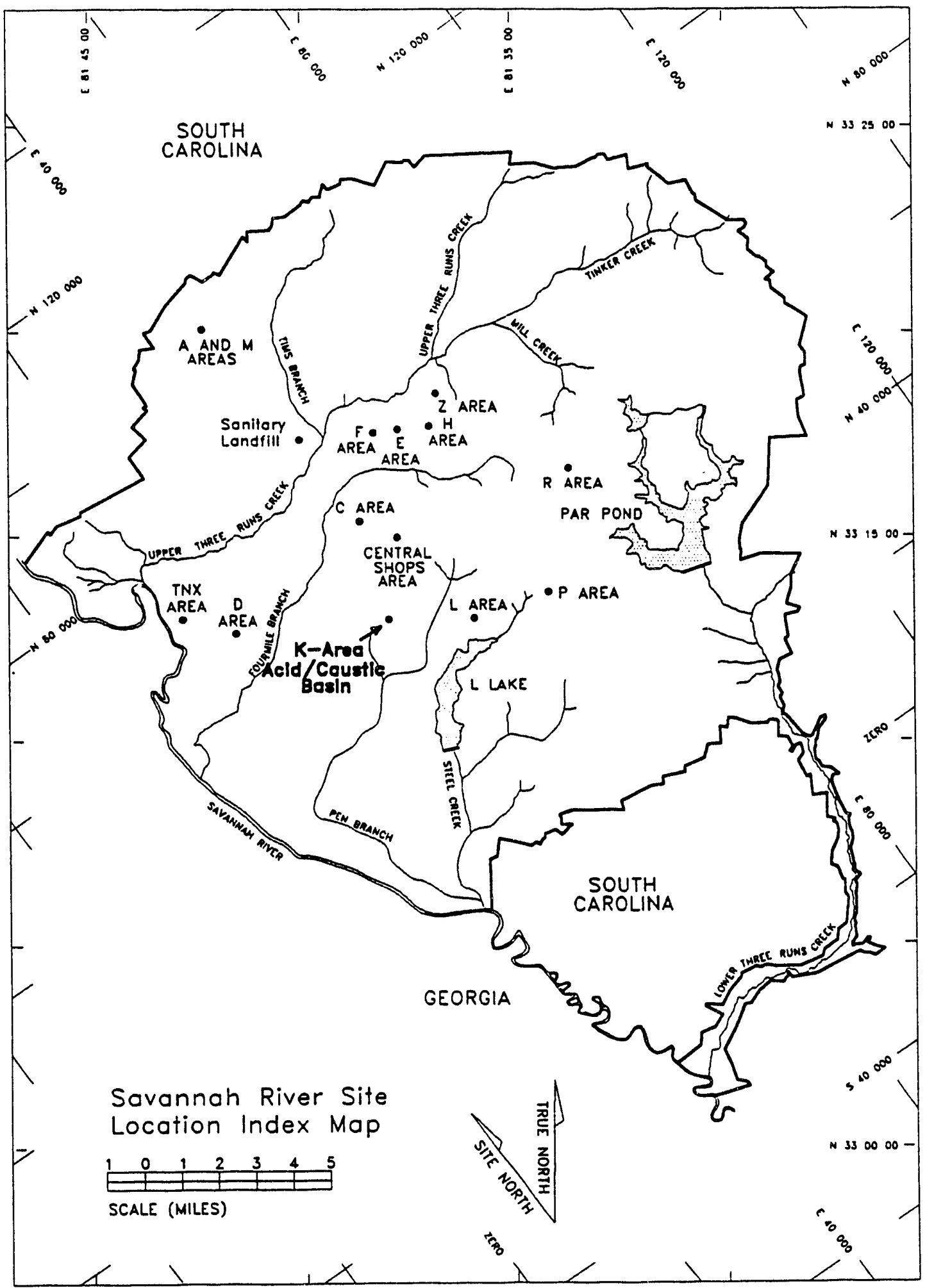

Figure 1. Location of the K-Area Acid/Caustic Basin at the Savannah River Site 


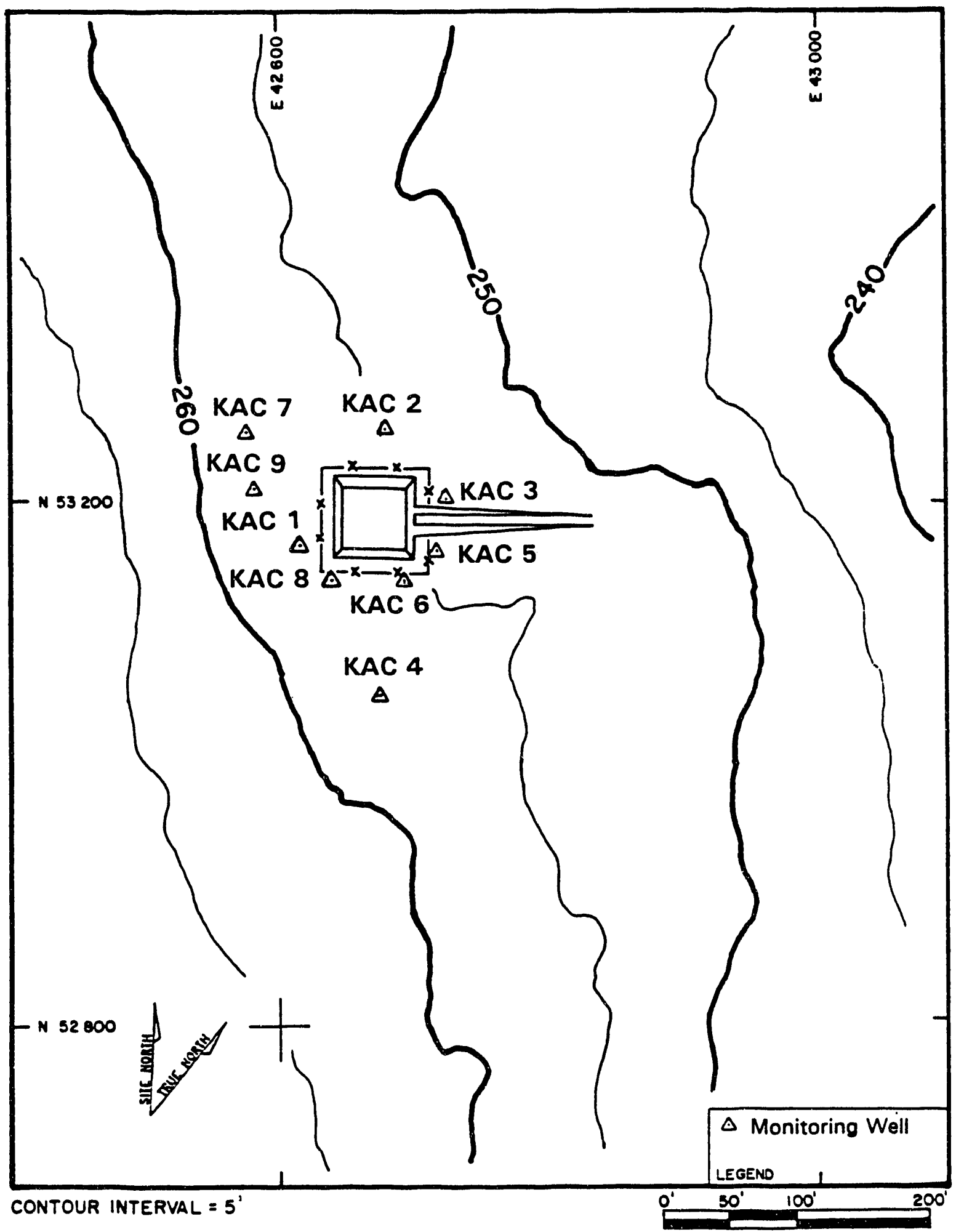

Figure 2. Location of Groundwater Monitoring Wells at the K-Area Acid/Caustic Basin 


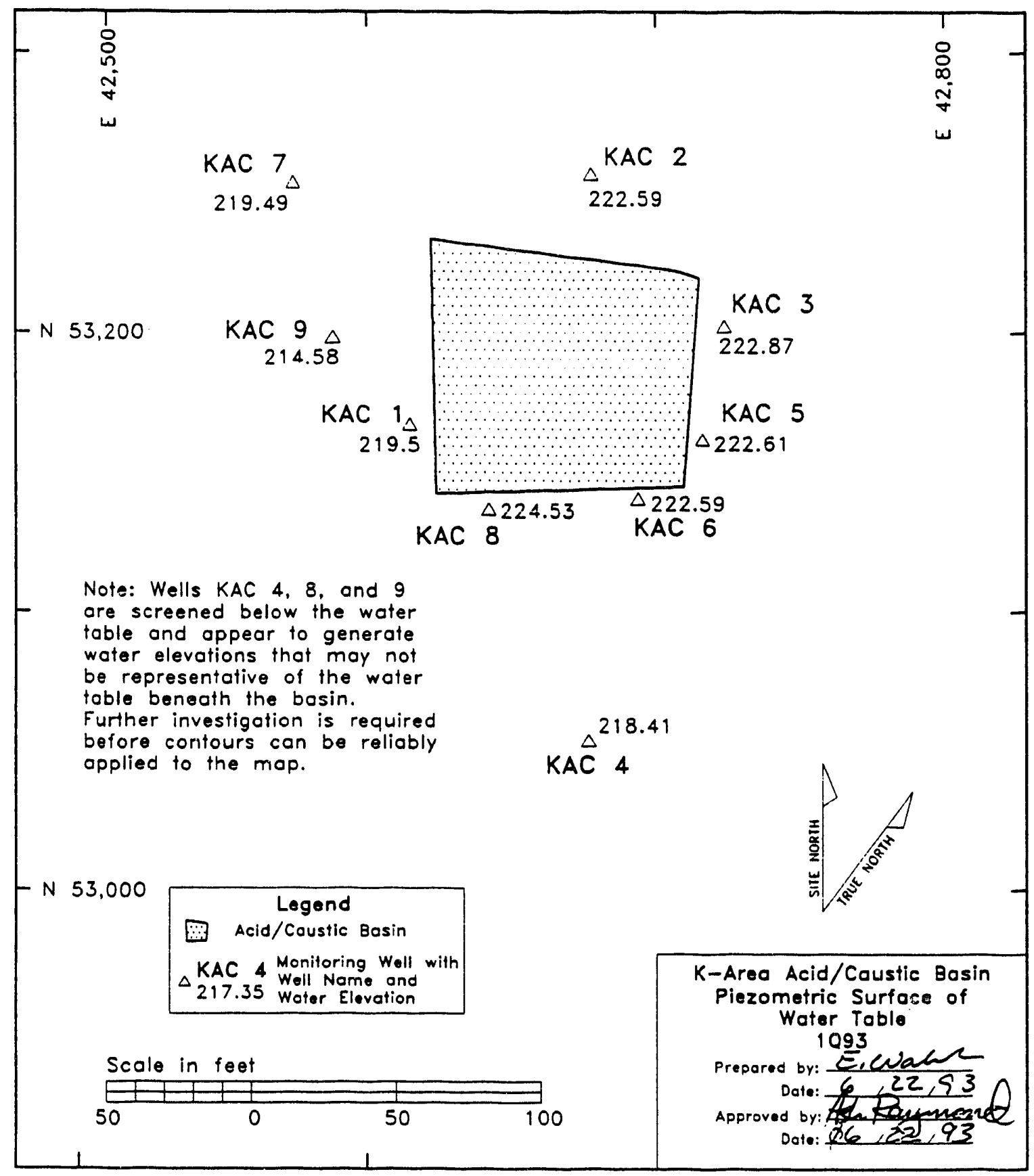

Figure 3. Water-Elevation Contour Map of the Water Table at the K-Area Acid/Caustic Basin 
WSRC-TR-93-277

Appendix D - Groundwater Monitoring Results Tables 


\section{Key to Reading the Tables}

The following abbreviations may appear in the tabular data:

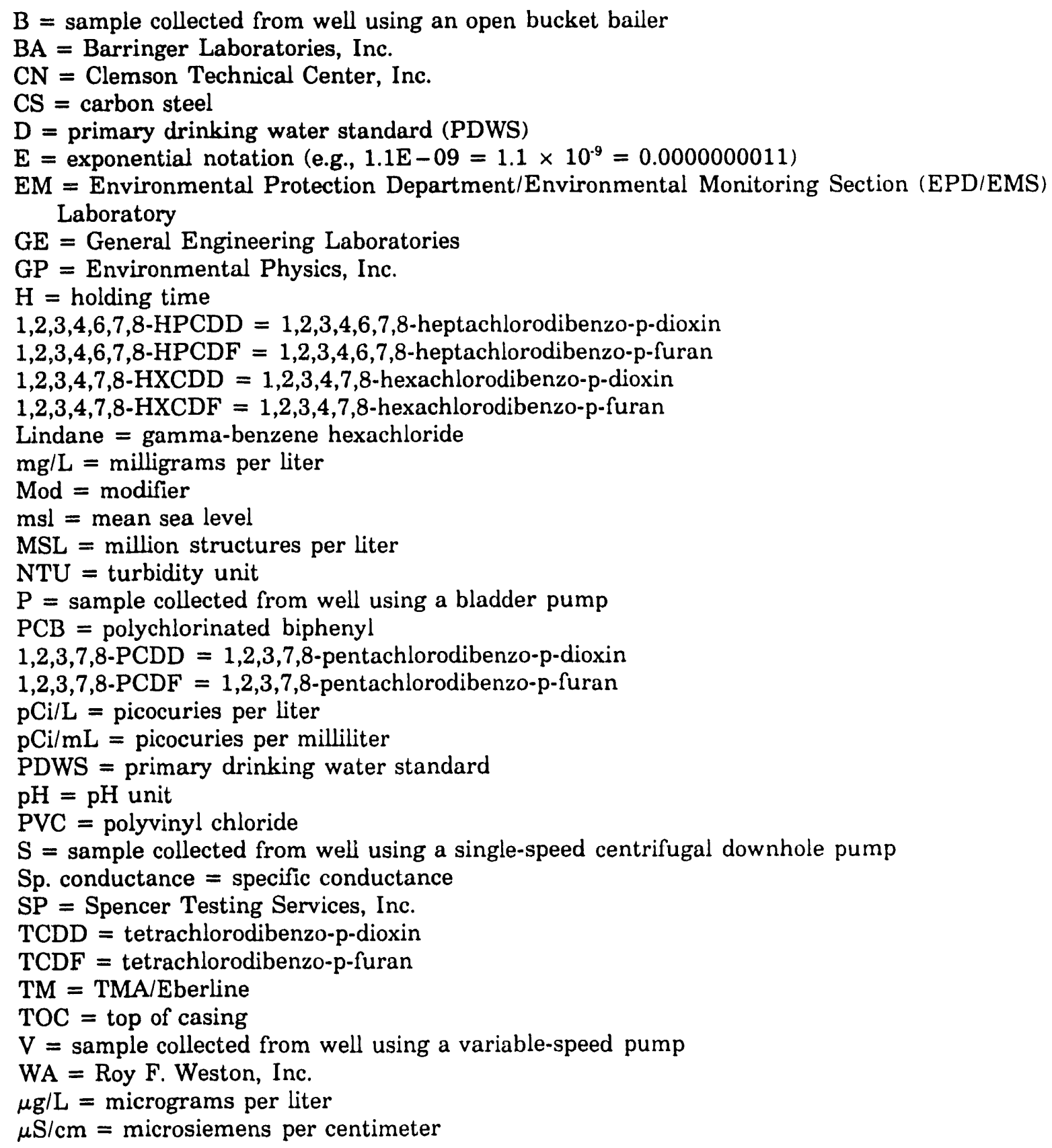




\section{Holding Times}

Standard analytical methods include a limit, called holding time, on the maximum elapsed time between sample collection and extraction or analysis by the laboratory. In the data tables, a large dot $(\bullet)$ in the $\mathrm{H}$ (holding time) column indicates that holding time was exceeded. Analyses performed beyond holding time may not yield valid results.

The South Carolina Department of Health and Environmental Control allows only 15 minutes to elapse between sampling and analysis for $\mathrm{pH}$. Thus, only field $\mathrm{pH}$ measurements can meet the holding time criterion; laboratory $\mathrm{pH}$ analyses will always exceed it.

Laboratory-initiated procedures for reducing the number of other analyses performed out of holding time include subcontracting analyses when difficulties with equipment, personnel, or work load would prevent timely analyses. SRS reduces the compensation to laboratories for analyses performed out of holding time.

\section{Data Qualification}

The contract laboratories continually assess their own accuracy and precision according to U.S. Environmental Protection Agency (EPA) guidelines. They submit sample- or batchspecific quality assurance/quality control information either at the same time as analytical results or in a quarterly summary. Properly defined and used result modifiers (also referred to as qualifiers) can be a key component in assessing data useability. Result modifiers designed by Environmental Protection Department/Environmental Monitoring Section and provided to the primary laboratories are defined below. These modifiers appear in the data tables under the column "Mod." The lettered modifiers are based on EPA's STORET codes.

Result modifier Definition

(Blank) Data are not qualified. Number should be interpreted exactly as reported.

A

$J$

L

M

R

$T$

V

$Y$

Value reported is the mean of two or more determinations.

Value is estimated because quantitation in the sample or in associated quality control samples did not meet specifications.

Value is off-scale high. The actual value is not known but is known to be greater than the value shown.

Presence of the analyte is verified but not quantified.

Result was rejected because performance requirements in the sample analysis or associated quality control analyses were not met.

Analyte was not detected; if present, it was below the criteria for detection.

Analyte was detected in an associated method blank.

Result was obtained from an unpreserved or improperly preserved sample. Data may not be accurate. 
Result modifier

1

2

3

4

6

\section{Definition}

Result mav be an underestimation of the true value due to analytical bias.

Result may be an overestimation of the true value due to analytical bias.

The associated result may be of poor precision (high variability) due to analytical bias.

Result is associated with QA results indicating matrix interference.

The associated result is from a reanalysis performed out of holding time due to problems with an earlier analysis. 
Table 1. Maximum Results for Constituents Exceeding Final Primary Drinking Water Standards

\begin{tabular}{|c|c|c|c|c|c|c|}
\hline Well & Constituent & $\underline{\text { Unit }}$ & $\underline{2092}$ & $\underline{3092}$ & $\underline{4092}$ & 1093 \\
\hline $\mathrm{N}^{\circ}$ & None & $N$ & $N$ & $N$ & $N$ & $N$ \\
\hline
\end{tabular}

Table 2. Maximum Results for Constituents Exceeding Half the Final Primary Drinking Water Standards, Other Flag 1 or Flag 2 Criteria, or the SRS Turbidity Standard

\begin{tabular}{|c|c|c|c|c|c|c|}
\hline Well & & Constituent & Unit & 1093 & Mod & Flag \\
\hline KAC & 2 & Specific conductance & $\mu \mathrm{S} / \mathrm{cm}$ & 378 & J & 1 \\
\hline \multirow[t]{2}{*}{ KAC } & 4 & Aluminum & $\mu g / L$ & 41 & & 1 \\
\hline & & Iron & $\mu g / L$ & 170 & v & 1 \\
\hline \multirow[t]{2}{*}{ KAC } & 5 & Aluminum & $\mu g / L$ & 30 & J3 & 1 \\
\hline & & Iron & $\mu g / L$ & 176 & $v$ & 1 \\
\hline \multirow[t]{2}{*}{ KAC } & 6 & Aluminum & $\mu g / L$ & 181 & & 2 \\
\hline & & Iron & $\mu g / L$ & 467 & V & 2 \\
\hline \multirow[t]{4}{*}{ KAC } & 7 & Specific conductance & $\mu \mathrm{S} / \mathrm{cm}$ & 310 & $\jmath$ & 1 \\
\hline & & Aluminum & $\mu g / L$ & 4,660 & & 2 \\
\hline & & Iron & $\mu g / L$ & 987 & $v$ & 2 \\
\hline & & Lead & $\mu g / L$ & 42 & & 2 \\
\hline \multirow[t]{4}{*}{ KAC } & 8 & Specific conductance & $\mu \mathrm{S} / \mathrm{cm}$ & 271 & J & 1 \\
\hline & & Aluminum & $\mu g / L$ & 101 & & 2 \\
\hline & & Iron & $\mu g / L$ & 475 & V & 2 \\
\hline & & Manganese & 吾 & 36 & & 1 \\
\hline \multirow[t]{4}{*}{ KAC } & 9 & $\mathrm{pH}$ & $\mathrm{pH}$ & 9.3 & J & 1 \\
\hline & & Specific conductance & $. \mathrm{S} / \mathrm{cm}$ & 324 & $J$ & 2 \\
\hline & & Aluminum & $\mu g / L$ & 403 & $\mathrm{Y}$ & 2 \\
\hline & & Sulfate & $\mu \mathrm{g} / \mathrm{L}$ & 326,000 & & 1 \\
\hline
\end{tabular}

Note: These res sits do not include field data results. 
Table 3. Groundwater Monitoring Results for Individual Wells

\section{WELL KAC 1}

\begin{tabular}{|c|c|c|c|c|c|c|}
\hline SRS Coord. & Lat/Longitude & Screen Zone Elevation & Top of Casing & Casing & Pump & Formation \\
\hline $\begin{array}{l}V 53167.0 \\
=42614.8\end{array}$ & $\begin{array}{l}33.212893^{\circ} \mathrm{N} \\
81.657866^{\circ} \mathrm{W}\end{array}$ & 22 & 2 & $C$ & V & \\
\hline
\end{tabular}

\section{FIELD MEASUREMENTS}

Sample date: 02/04/93

Depth to water: $46.50 \mathrm{ft}(14.17 \mathrm{~m})$ below TOC

Water elevation: $219.50 \mathrm{ft}(66.90 \mathrm{~m}) \mathrm{msl}$

Sp. conductance: $95 \mu \mathrm{S} / \mathrm{cm}$

Water evacliated before sampling: $33 \mathrm{gal}$

\section{LABORATORY ANALYSES}

$\underline{H}$ D Analyte

$\mathrm{pH}$

$\mathrm{pH}$

Specific conductance

Specific conductance

Turbidity

Turbidity

Aluminum

Arsenic

Barium

Cadmium

Calcium

Chloride

Chromium

2,4-Dichlorophenoxyacetic acid

Endrin

Endrin

Fluoride

Iron

Lead

Lindane

Lindane

Magnesium

Manganese

Niercury

Mercury

Methoxychlor

Methoxychlor

Methoxychlor

Nitrate as nitrogen

Phenols

Potassium

Selenium

Silica

Silver

Sodium

Sulfate

Sulfate

Total dissolved solids

Total organic carbon
Result

5.8
5.7

95

0.70

0.69

$<20$

$<2.0$

$<4.0$

$<2.0$

107

5,450

$<4.0$

$<1.1$

$<0.11$

$<0.22$

$<100$

13

$<3.0$

$<0.056$

$<0.11$
50

$<2.0$

$<0.20$

$<0.20$

$<0.56$

$<1.1$

$<1.1$

481

$<5.0$

$<500$

$<2.0$

2,880

$<2.0$

13,500

12,100

12,300

$<1,000$

$<1,000$
Time: $14: 12$

$\mathrm{pH}: 6.0$

Alkalinity: $17 \mathrm{mg} / \mathrm{L}$

Water temperature: $19.7{ }^{\circ} \mathrm{C}$

Volumes purged: 2.5 well volumes 
WELL KAC 1 collected on 02/04/93, laboratory analyses (cont.)

\begin{tabular}{|c|c|c|c|c|c|c|}
\hline \multirow[t]{17}{*}{$\underline{D}$} & Analyte & Result & \multirow[t]{17}{*}{$\underline{\text { Mod }}$} & Unit & Flag & $\underline{L a b}$ \\
\hline & Total organic halogens & $<5.0$ & & $\mu g / L$ & 0 & WA \\
\hline & Total organic halogens & $<5.0$ & & $\mu \mathrm{g} / \mathrm{L}$ & 0 & WA \\
\hline & Total phosphates las PI & $<50$ & & $\mu \mathrm{g} / \mathrm{L}$ & 0 & WA \\
\hline & Toxaphene & $<1.1$ & & $\mu \mathrm{g} / \mathrm{L}$ & 0 & WA \\
\hline & Toxaphene & $<2.2$ & & $\mu \mathrm{g} / \mathrm{L}$ & 0 & NA \\
\hline & Toxaphene & $<2.2$ & & $\mu \mathrm{g} / \mathrm{L}$ & 0 & WA \\
\hline & 2,4,5-TP (Silvex) & $<0.54$ & & $\mu \mathrm{g} / \mathrm{L}$ & 0 & WA \\
\hline & Gross alpha & 7.OE-01 + 7.0E-01 & & $\mathrm{pCi} / \mathrm{L}$ & 0 & TM \\
\hline & $\begin{array}{l}\text { Gross alpha } \\
\text { Nonvolatile beta }\end{array}$ & $\begin{array}{l}<4.0 \mathrm{E}-01 \\
1.1 \mathrm{E}+00 \pm 1.4 \mathrm{E}+00\end{array}$ & & $\begin{array}{l}\mathrm{pCi} / \mathrm{L} \\
\mathrm{pCi} / \mathrm{L}\end{array}$ & $\begin{array}{l}0 \\
0\end{array}$ & $\begin{array}{l}\text { TM } \\
\text { TM }\end{array}$ \\
\hline & Nonvolatile beta & $1.0 E-01 \pm 1.4 E+00$ & & $\mathrm{pCi} / \mathrm{L}$ & 0 & $T M$ \\
\hline & Radium-226 & $<2.0 \mathrm{E} \cdot 01$ & & $\mathrm{pCi} / \mathrm{L}$ & 0 & TM \\
\hline & Radium-226 & $<2.8 \mathrm{E}-01$ & & $\mathrm{pCi} / \mathrm{L}$ & 0 & TM \\
\hline & Radium-228 & $7.0 \mathrm{E}-01 \pm 9.0 \mathrm{E}-01$ & & $\mathrm{pCi} / \mathrm{L}$ & 0 & TM \\
\hline & Radium-228 & $8.0 \mathrm{E}-01 \pm 8.0 \mathrm{E}-01$ & & $\mathrm{pCi} / \mathrm{L}$ & 0 & TM \\
\hline & Tritium & $1.9 \mathrm{E}+00 \pm 7.6 \mathrm{E}-01$ & & $\mathrm{pCi} / \mathrm{mL}$ & 0 & \\
\hline & Tritium & $1.9 E+00 \pm 6.4 E-01$ & & $\mathrm{pCi} / \mathrm{mL}$ & 0 & TM \\
\hline
\end{tabular}

\section{WELL KAC 2}

\begin{tabular}{|c|c|c|c|c|c|c|}
\hline SRS Coord. & Lat/Longitude & Screen Zone Elevation & Top of Casing & $\underline{\text { Casing }}$ & Pump & Formation \\
\hline $\begin{array}{l}53255.5 \\
42677.2\end{array}$ & $\begin{array}{l}33.213191{ }^{\circ} \mathrm{N} \\
81.657873^{\circ} \mathrm{W}\end{array}$ & $225.4-195.4$ & $257.5 \mathrm{ft} \mathrm{msl}$ & 4" PVC & S & \\
\hline
\end{tabular}

\section{FIELD MEASUREMENTS}

Sample date: 02/04/93

Depth to water: $34.91 \mathrm{ft}(10.64 \mathrm{~m})$ below TOC

Water elevation: $222.59 \mathrm{ft}(67.85 \mathrm{~m}) \mathrm{msl}$

Sp. conductance: $387 \mu \mathrm{S} / \mathrm{cm}$

Water evacuated before sampling: $66 \mathrm{gal}$

\section{LABORATORY ANALYSES}

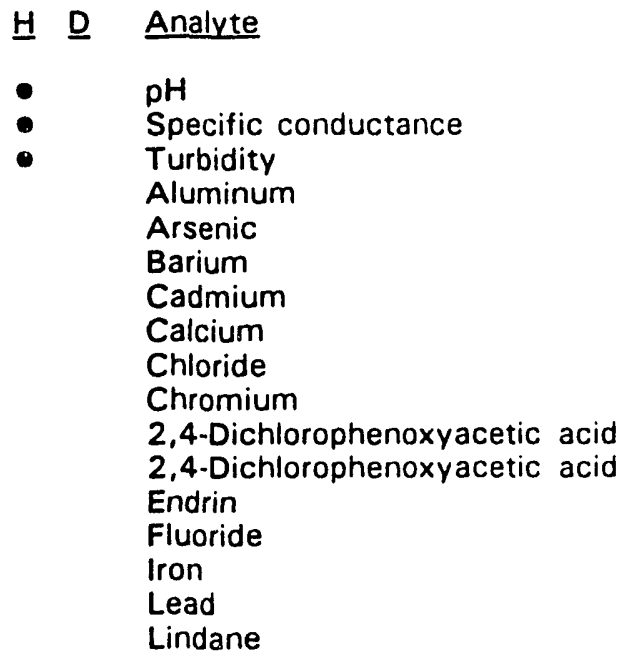

Time: $11: 26$

pH: 6.4

Alkalinity: $33 \mathrm{mg} / \mathrm{L}$

Water temperature: $20.1^{\circ} \mathrm{C}$

Volumes purged: 3.7 well volumes

\begin{tabular}{|c|c|c|c|}
\hline Result & Mod & $\underline{\text { Unit }}$ & Flag \\
\hline 6.0 & $J$ & $\mathrm{pH}$ & 0 \\
\hline 378 & $\mathrm{~J}$ & $\mu \mathrm{S} / \mathrm{cm}$ & 1 \\
\hline 14 & J & NTU & 0 \\
\hline$<20$ & & $\mu g / L$ & 0 \\
\hline$<2.0$ & & $\mu \mathrm{g} / \mathrm{L}$ & 0 \\
\hline$<4.0$ & & $\mu \mathrm{g} / \mathrm{L}$ & 0 \\
\hline$<2.0$ & & $\mu \mathrm{g} / \mathrm{L}$ & 0 \\
\hline 223 & v & $\mu \mathrm{g} / \mathrm{L}$ & 0 \\
\hline 5,870 & & $\mu \mathrm{g} / \mathrm{L}$ & 0 \\
\hline$<4.0$ & & $\mu \mathrm{g} / \mathrm{L}$ & 0 \\
\hline$<1.1$ & & $\mu \mathrm{g} / \mathrm{L}$ & 0 \\
\hline$<2.2$ & & $\mu \mathrm{g} / \mathrm{L}$ & 0 \\
\hline$<0.91$ & & $\mu \mathrm{g} / \mathrm{L}$ & 0 \\
\hline$<100$ & & $\mu \mathrm{g} / \mathrm{L}$ & 0 \\
\hline 87 & v & $\mu \mathrm{g} / \mathrm{L}$ & 0 \\
\hline$<3.0$ & & $\mu \mathrm{g} / \mathrm{L}$ & 0 \\
\hline$<0.056$ & & $\mu \mathrm{g} / \mathrm{L}$ & 0 \\
\hline
\end{tabular}

$\overline{- \text { = exceeded holding time. }}$ = exceeded final primary drinking water standard. 
WELL KAC 2 collected on 02/04/93, laboratory analyses (cont.)

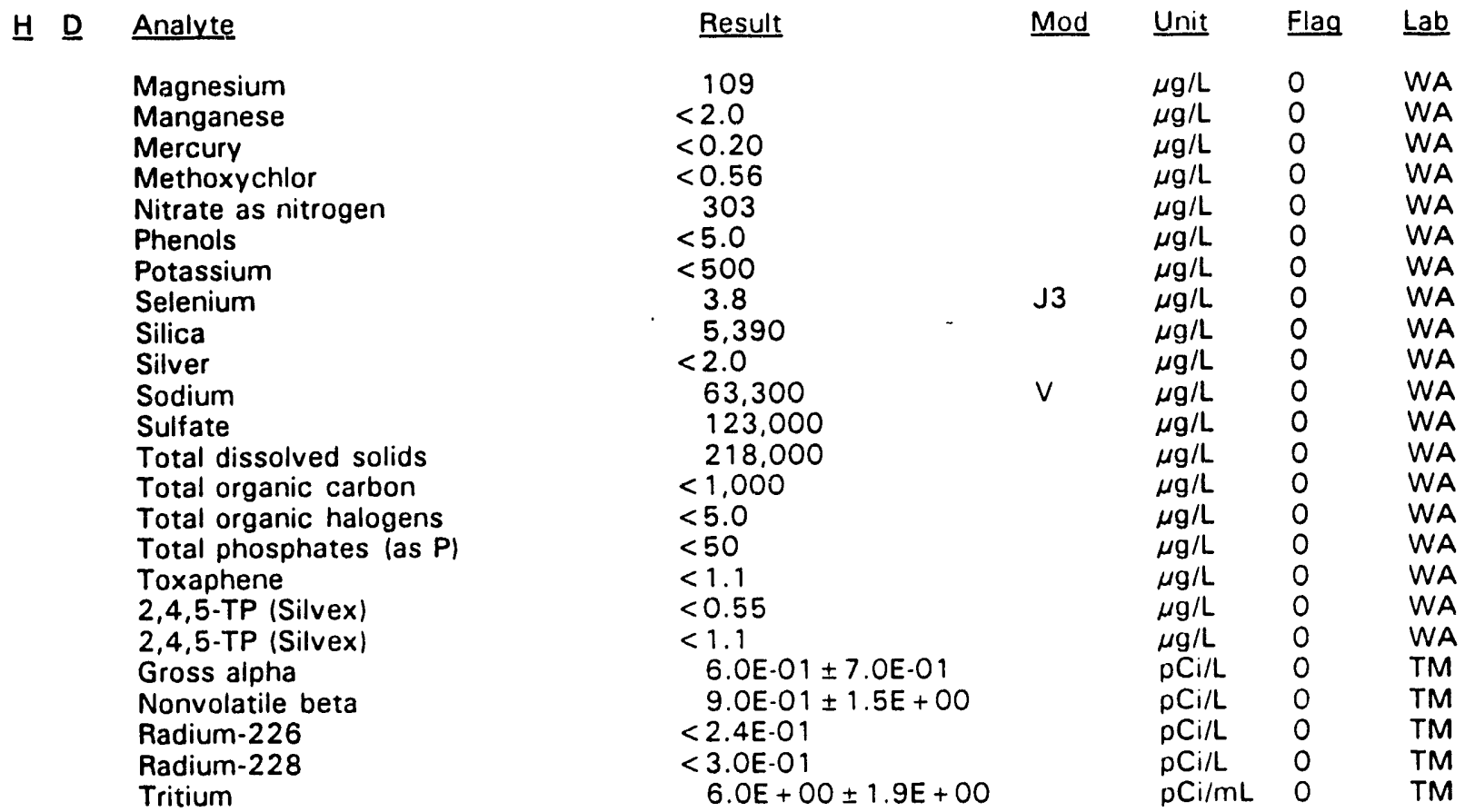

\section{WELL KAC 3}

\begin{tabular}{|c|c|c|c|c|c|c|}
\hline SRS Coord. & Lat/Longitude & Screen Zone Elevation & Top of Casing & Casing & Puimp & Formation \\
\hline $\begin{array}{l}53201.8 \\
.2723 .9\end{array}$ & $\begin{array}{l}33.213148^{\circ} \mathrm{N} \\
81.657646^{\circ} \mathrm{W}\end{array}$ & $225.8-195.8 \mathrm{ft} \mathrm{msl}$ & $257.8 \mathrm{ft} \mathrm{msl}$ & 4" PVC & $S$ & \\
\hline
\end{tabular}

\section{FIELD MEASUREMENTS}

Sample date: $02 / 04 / 93$

Depth to water: $34.93 \mathrm{ft}(10.65 \mathrm{~m})$ below TOC Water elevation: $222.87 \mathrm{ft}(67.93 \mathrm{~m}) \mathrm{msl}$

Sp. conductance: $230 \mu \mathrm{S} / \mathrm{cm}$

Water evacuated before sampling: $131 \mathrm{gal}$

\section{LABORATORY ANALYSES}

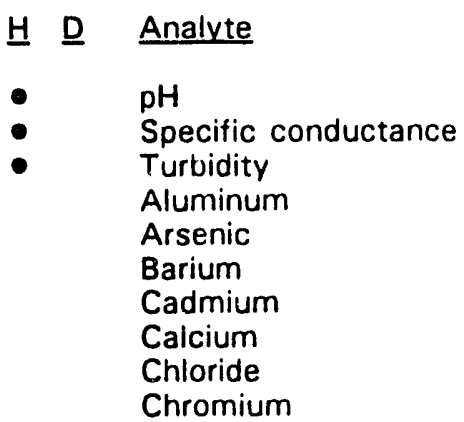

$$
\begin{aligned}
& \text { Result } \\
& 6.0 \\
& 227 \\
0.64 & < \\
< & 20 \\
< & 2.0 \\
< & 4.0 \\
< & 2.0 \\
& 825 \\
& 5.980 \\
< & 4.0
\end{aligned}
$$

Time: $11: 50$

$\mathrm{pH}: 6.4$

Alkalinity: $37 \mathrm{mg} / \mathrm{L}$

Water temperature: $19.0^{\circ} \mathrm{C}$

Volumes purged: 7.4 well volumes

\footnotetext{
a = exceeded holding time. $=$ = exceeded final primary drinking water standard.
} 
WELL KAC 3 collected on 02/04/93, laboratory analyses (cont.)

\begin{tabular}{|c|c|c|c|c|c|c|}
\hline \multirow[t]{31}{*}{$\underline{H} \underline{D}$} & Analyte & Result & $\underline{\text { Mod }}$ & $\underline{\text { Unit }}$ & Flag & $\underline{\text { Lab }}$ \\
\hline & 2,4-Dichlorophenoxyacetic acid & $<1.1$ & & $\mu \mathrm{g} / \mathrm{L}$ & 0 & WA \\
\hline & Endrin & $<0.11$ & & $\mu \mathrm{g} / \mathrm{L}$ & 0 & \\
\hline & Fluoride & $<100$ & & $\mu \mathrm{g} / \mathrm{L}$ & 0 & WA \\
\hline & Fluoride & $<100$ & & $\mu \mathrm{g} / \mathrm{L}$ & 0 & WA \\
\hline & Iron & 12 & $J 3 \mathrm{~V}$ & $\mu \mathrm{g} / \mathrm{L}$ & 0 & WA \\
\hline & Lead & $<3.0$ & & $\mu \mathrm{g} / \mathrm{L}$ & 0 & WA \\
\hline & Lindane & $<0.055$ & & $\mu \mathrm{g} / \mathrm{L}$ & 0 & WA \\
\hline & Magnesium & 273 & & $\mu \mathrm{g} / \mathrm{L}$ & 0 & WA \\
\hline & Manganese & $<2.0$ & & $\mu \mathrm{g} / \mathrm{L}$ & 0 & WA \\
\hline & Mercury & $<0.20$ & & $\mu \mathrm{g} / \mathrm{L}$ & 0 & WA \\
\hline & Methoxychlor & $<0.55$ & & $\mu \mathrm{g} / \mathrm{L}$ & 0 & WA \\
\hline & Nitrate as nitrogen & 145 & & $\mu \mathrm{g} / \mathrm{L}$ & 0 & WA \\
\hline & Phenols & $<5.0$ & & $\mu \mathrm{g} / \mathrm{L}$ & 0 & WA \\
\hline & Potassium & 591 & J3 & $\mu \mathrm{g} / \mathrm{L}$ & 0 & WA \\
\hline & Selenium & $<2.0$ & & $\mu \mathrm{g} / \mathrm{L}$ & 0 & WA \\
\hline & Silica & 5,200 & & $\mu \mathrm{g} / \mathrm{L}$ & 0 & WA \\
\hline & Silver & $<2.0$ & & $\mu \mathrm{g} / \mathrm{L}$ & 0 & WA \\
\hline & Sodium & 38,500 & $\mathrm{v}$ & $\mu \mathrm{g} / \mathrm{L}$ & 0 & WA \\
\hline & Sulfate & 57,800 & & $\mu \mathrm{g} / \mathrm{L}$ & 0 & WA \\
\hline & Total dissolved solids & 133,000 & & $\mu \mathrm{g} / \mathrm{L}$ & 0 & WA \\
\hline & Total organic carbon & $<1,000$ & & $\mu \mathrm{g} / \mathrm{L}$ & 0 & WA \\
\hline & Total organic halogens & $<5.0$ & & $\mu \mathrm{g} / \mathrm{L}$ & 0 & WA \\
\hline & Total phosphates (as P) & $<50$ & & $\mu \mathrm{g} / \mathrm{L}$ & 0 & WA \\
\hline & Toxaphene & $<1.1$ & & $\mu \mathrm{g} / \mathrm{L}$ & 0 & WA \\
\hline & 2,4,5-TP (Silvex) & $<0.54$ & & $\mu g / L$ & 0 & WA \\
\hline & Gross alpha & $5.0 \mathrm{E}-01 \pm 7.0 \mathrm{E}-01$ & & $\mathrm{pCi} / \mathrm{L}$ & 0 & TM \\
\hline & Nonvolatile beta & $2.1 E+00 \pm 1.6 E+00$ & & $\mathrm{pCi} / \mathrm{L}$ & 0 & TM \\
\hline & Radium-226 & $<1.9 \mathrm{E}-01$ & & $\mathrm{pCi} / \mathrm{L}$ & 0 & $T M$ \\
\hline & Radium-228 & $<3.0 \mathrm{E}-01$ & & $\mathrm{pCi} / \mathrm{L}$ & 0 & TM \\
\hline & Tritium & $3.1 \mathrm{E}+00 \pm 9.8 \mathrm{E}-01$ & & $\mathrm{pCl} / \mathrm{mL}$ & 0 & TM \\
\hline
\end{tabular}

\section{WELL KAC 4}

\begin{tabular}{|c|c|c|c|c|c|c|}
\hline SRS Coord. & Lat/Longitude & Screen Zone Elevation & Top of Casing & Casing & Pump & Formation \\
\hline $\begin{array}{l}15305 \\
4267\end{array}$ & $\begin{array}{l}33.212743^{\circ} \mathrm{N} \\
81.657484^{\circ} \mathrm{W}\end{array}$ & $208.0 \cdot 17$ & 260 & 4" PVC & S & \\
\hline
\end{tabular}

\section{FIELD MEASUREMENTS}

Sample date: 02/04/93

Depth to water: $41.59 \mathrm{ft}(12.68 \mathrm{~m})$ below TOC

Water elevation: $218.41 \mathrm{ft}(66.57 \mathrm{~m}) \mathrm{msl}$

Sp. conductance: $75 \mu \mathrm{S} / \mathrm{cm}$

Water evacuated before sampling: $139 \mathrm{gal}$

\section{LABORATORY ANALYSES}

\begin{tabular}{|c|c|c|c|c|c|}
\hline$\underline{H}$ D & Analvte & Result & Mod & Unit & Flag \\
\hline : & $\begin{array}{l}\mathrm{pH} \\
\mathrm{pH} \\
\mathrm{pH} \\
\mathrm{pH}\end{array}$ & $\begin{array}{l}5.6 \\
5.6 \\
5.2 \\
5.3\end{array}$ & $\begin{array}{l}J \\
J \\
J\end{array}$ & $\begin{array}{l}\mathrm{pH} \\
\mathrm{pH} \\
\mathrm{pH} \\
\mathrm{pH}\end{array}$ & $\begin{array}{l}0 \\
0 \\
0 \\
0\end{array}$ \\
\hline
\end{tabular}

Time: $13: 28$

pH: 5.1

Water temperature: $18.7^{\circ} \mathrm{C}$
Alkalinity: $1 \mathrm{mg} / \mathrm{L}$

Volumes purged: 5.2 well volumes

$\overline{0}=$ exceeded huiding time. $=$ = exceeded final primary drinking water standard. 
WELL KAC 4 collected on 02/04/93, laboratory analyses (cont.)

H D Analyte

Specific conductance

Specific conductance

- Specific conductance

- Specific conductance

Turbidity

Turbidity

- Turbidity

- Turbidity

Aluminum

Aluminum

Aluminum

Aluminum

Arsenic

Arsenic

Arsenic

Arsenic

Barium

Barium

Barium

Barium

Cadmium

Cadmium

Cadmium

Cadmium

Calcium

Calcium

Calcium

Calcium

- Chloride

Chloride

Chloride

Chloride

Chromium

Chromium

Chromium

Chromium

2,4-Dichlorophenoxyacetic acid

2,4-Dichlorophenoxyacetic acid

2,4-Dichlorophenoxyacetic acid

2,4-Dichlorophenoxyacetic acid

Endrin

Endrin

Endrin

Endrin

Fluoride

Fluoride

Fluoride

Fluoride

Iron

Iron

Iron

Iron

Lead

Lead

Lead

Lead

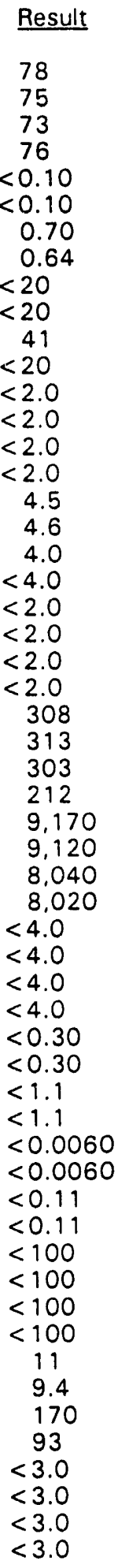

Mod Unit Flag Lab

$\begin{array}{llll} & \mu \mathrm{S} / \mathrm{cm} & 0 & \mathrm{GE} \\ & \mu \mathrm{S} / \mathrm{cm} & 0 & \mathrm{GE} \\ \mathrm{J} & \mu \mathrm{S} / \mathrm{cm} & 0 & \text { WA } \\ \mathrm{J} & \mu \mathrm{S} / \mathrm{cm} & 0 & \text { WA } \\ & \text { NTU } & 0 & \text { GE } \\ & \text { NTU } & 0 & \text { GE }\end{array}$

J NTU 0 WA

$J$ NTU 0 WA

$\mu \mathrm{g} / \mathrm{L} \quad \mathrm{O} \quad \mathrm{GE}$

$\mu \mathrm{g} / \mathrm{L} \quad \mathrm{O} \quad \mathrm{GE}$

$\mu \mathrm{g} / \mathrm{L} \quad 1 \quad$ WA

$\mu \mathrm{g} / \mathrm{L} \quad 0 \quad$ WA

$\mu \mathrm{g} / \mathrm{L} \quad \mathrm{O} \quad \mathrm{GE}$

$\mu \mathrm{g} / \mathrm{L} \quad 0 \quad \mathrm{GE}$

$\mu \mathrm{g} / \mathrm{L} \quad \mathrm{W}$ WA

$\mu g / L \quad 0 \quad$ WA

$\mu \mathrm{g} / \mathrm{L} \quad \mathrm{O} \quad \mathrm{GE}$

$\mu \mathrm{g} / \mathrm{L} \quad \mathrm{O} \quad \mathrm{GE}$

J3 $\mu \mathrm{g} / \mathrm{L} \quad 0 \quad$ WA

$\mu \mathrm{g} / \mathrm{L} \quad \mathrm{O} \quad$ WA

$\mu \mathrm{g} / \mathrm{L} \quad \mathrm{O} \quad \mathrm{GE}$

$\mu g / L \quad O \quad$ GE

$\mu \mathrm{g} / \mathrm{L} \quad \mathrm{O} \quad$ WA

$\mu \mathrm{g} / \mathrm{L} \quad \mathrm{O}$ WA

$J 2 \mu g / L \quad 0 \quad$ GE

J2 $\mu \mathrm{g} / \mathrm{L} \quad 0 \quad \mathrm{GE}$

$V \quad \mu g / L \quad 0 \quad$ WA

$\begin{array}{llll}V & \mu g / L & 0 & W A\end{array}$

J6 $\mu \mathrm{g} / \mathrm{L} \quad 0 \quad \mathrm{GE}$

J6 $\mu g / L \quad 0 \quad$ GE

WA

WA

GE

GE

WA

WA

GE

GE

WA

WA

GE

GE

WA

WA

GE

GE

WA

WA

GE

GE

WA

WA

GE

GE

WA

WA

$\overline{0=\text { exceeded holding time. }} \mathbf{0}=$ exceeded final primary drinking water standard. 
WELL KAC 4 collected on 02/04/93, laboratory analyses (cont.)

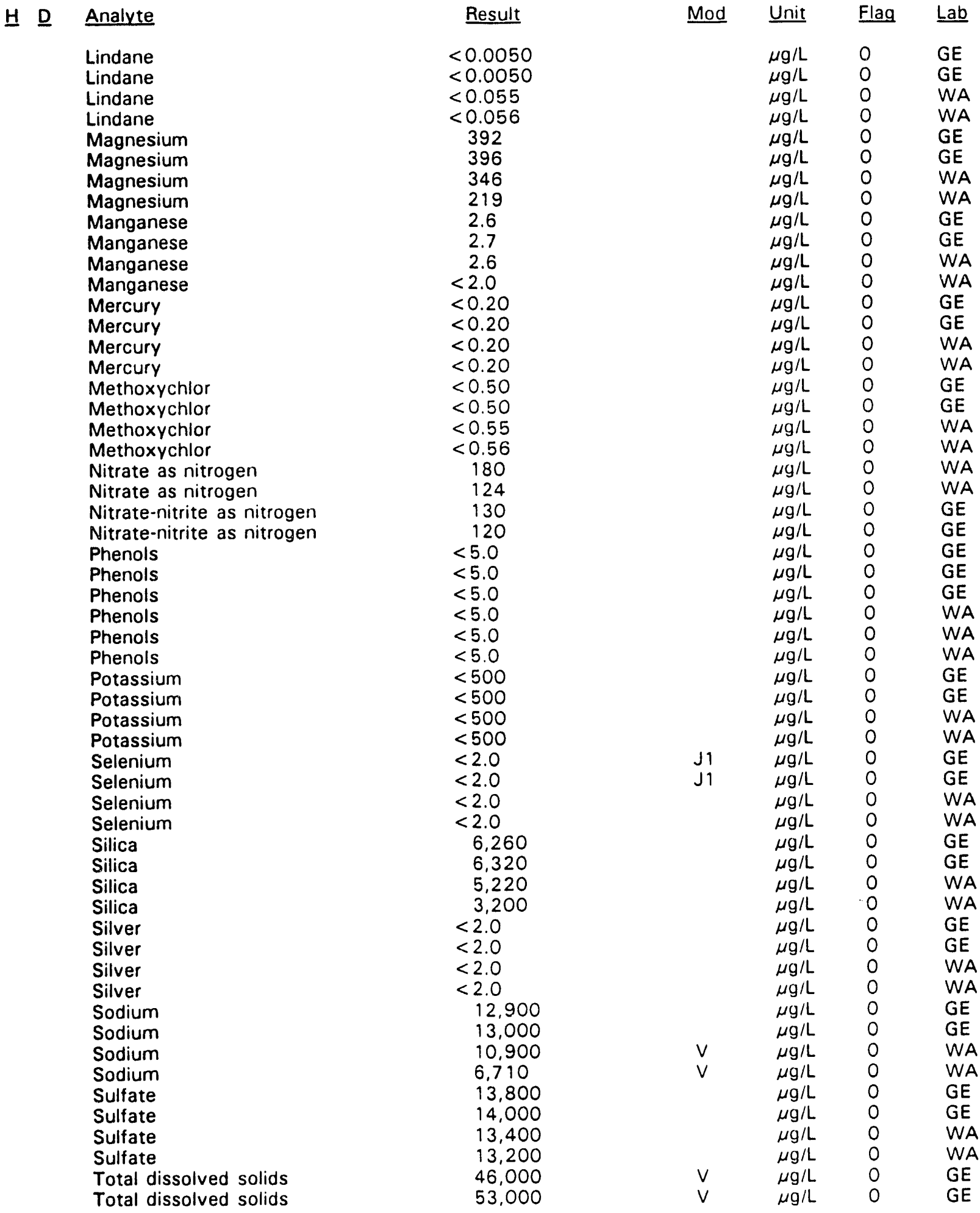

$\overline{0 \text { = exceeded holding time. }}$ = exceeded final prumary drınkıng water standard. 
WELL KAC 4 collected on 02/04/93, laboratory analyses (cont.)

H $\underline{\text { Analyte }}$

Total dissolved solids

Total dissolved solids

Total organic carbon

Total organic carbon

Total organic carbon

Total organic carbon

Total organic halogens

Total organic halogens

Total organic halogens

Total organic halogens

Total phosphates (as P)

Total phosphates (as P)

Total phosphates (as P)

Total phosphates (as P)

Toxaphene

Toxaphene

Toxaphene

Toxaphene

2,4,5-TP (Silvex)

2,4,5-TP (Silvex)

2,4,5-TP (Silvex)

2,4,5-TP (Silvex)

Gross alpha

Gross alpha

Gross alpha

Gross alpha

Nonvolatile beta

Nonvolatile beta

Nonvolatile beta

Nonvolatile beta

Radium-226

Radium-226

Radium-228

Radium-228

Radium, total alpha-emitting

Radium, total alpha-emitting

Tritium

Tritium

Tritium

Tritium

Tritium

$\begin{aligned} & \text { Result } \\ & 49.000 \\ & 60.000 \\ &< 1.000 \\ &< 1.000 \\ &< 1.000 \\ &<1,000 \\ &<5.0 \\ &<5.0 \\ &<5.0 \\ &<5.0 \\ &<50 \\ &<50 \\ &<50 \\ &<50 \\ &<0.24 \\ &<0.24 \\ &<1.1 \\ &<1.1 \\ &<0.090 \\ &<0.090 \\ &<0.54 \\ &<0.54 \\ &<2.0 E+00 \\ &<2.0 E+00 \\ & 1.4 E+00 \pm 8.0 E-01 \\ & 1.3 E+00 \pm 7.0 E-01 \\ &<2.0 E+00 \\ &<2.0 E+00 \\ & 1.2 E+00 \pm 1.4 E+00 \\ & 1.2 E+00 \pm 1.4 E+00 \\ & 3.0 E-01 \pm 2.2 E-01 \\ & 3.9 E-01 \pm 2.2 E-01 \\ & 8.0 E-01 \pm 8.0 E-01 \\ & 7.0 E-01 \pm 8.0 E-01 \\ & 1.1 E+00 \pm 9.0 E-01 \\ & 1.1 E+00 \pm 9.0 E-01 \\ & 8.3 E-01 \pm 3.0 E-01 \\ & 1.0 E+00 \pm 3.0 E-01 \\ & 1.3 E+00 \pm 3.0 E-01 \\ & 1.7 E+00 \pm 5.8 E-01 \\ & 2.1 E+00 \pm 6.7 E-01\end{aligned}$

\begin{tabular}{|c|c|c|c|}
\hline Mod & Unt & Flag & $\mathrm{Lab}$ \\
\hline & $\mu \mathrm{g} / \mathrm{L}$ & 0 & WA \\
\hline & $\mu \mathrm{g} / \mathrm{L}$ & 0 & WA \\
\hline & $\mu \mathrm{g} / \mathrm{L}$ & 0 & GE \\
\hline & $\mu \mathrm{g} / \mathrm{L}$ & 0 & GE \\
\hline & $\mu \mathrm{g} / \mathrm{L}$ & 0 & WA \\
\hline & $\mu g / L$ & 0 & WA \\
\hline & $\mu \mathrm{g} / \mathrm{L}$ & 0 & GE \\
\hline & $\mu \mathrm{g} / \mathrm{L}$ & 0 & $\mathrm{GE}$ \\
\hline & $\mu \mathrm{g} / \mathrm{L}$ & 0 & WA \\
\hline & $\mu \mathrm{g} / \mathrm{L}$ & 0 & WA \\
\hline & $\mu \mathrm{g} / \mathrm{L}$ & 0 & GE \\
\hline & $\mu \mathrm{g} / \mathrm{L}$ & 0 & $\overrightarrow{G E}$ \\
\hline & $\mu \mathrm{g} / \mathrm{L}$ & 0 & WA \\
\hline & $\mu \mathrm{g} / \mathrm{L}$ & 0 & WA \\
\hline & $\mu \mathrm{g} / \mathrm{L}$ & 0 & $\mathrm{GE}$ \\
\hline & $\mu \mathrm{g} / \mathrm{L}$ & 0 & $\overline{G E}$ \\
\hline & $\mu \mathrm{g} / \mathrm{L}$ & 0 & WA \\
\hline & $\mu \mathrm{g} / \mathrm{L}$ & 0 & WA \\
\hline & $\mu \mathrm{g} / \mathrm{L}$ & 0 & GE \\
\hline & $\mu \mathrm{g} / \mathrm{L}$ & 0 & GE \\
\hline & $\mu \mathrm{g} / \mathrm{L}$ & 0 & WA \\
\hline & $\mu g / L$ & 0 & WA \\
\hline & $p C i / L$ & 0 & GE \\
\hline & $\mathrm{pCi} / \mathrm{L}$ & 0 & GE \\
\hline & $\mathrm{pCi} / \mathrm{L}$ & 0 & TM \\
\hline & $\mathrm{pCi} / \mathrm{L}$ & 0 & TM \\
\hline & $\mathrm{pCi} / \mathrm{L}$ & 0 & GE \\
\hline & $\mathrm{pCi} / \mathrm{L}$ & 0 & GE \\
\hline & $\mathrm{pCi} / \mathrm{L}$ & 0 & $T M$ \\
\hline & $\mathrm{pCi} / \mathrm{L}$ & 0 & TM \\
\hline & $\mathrm{pCi} / \mathrm{L}$ & 0 & TM \\
\hline & $p \mathrm{Ci} / \mathrm{L}$ & 0 & TM \\
\hline & $\mathrm{pCi} / \mathrm{L}$ & 0 & TM \\
\hline & $\mathrm{pCi} / \mathrm{L}$ & 0 & TM \\
\hline & $\mathrm{pCi} / \mathrm{L}$ & 0 & GE \\
\hline & $\mathrm{pCi} / \mathrm{L}$ & 0 & GE \\
\hline & $\mathrm{pCi} / \mathrm{mL}$ & 0 & $\mathrm{GE}$ \\
\hline & $\mathrm{pCi} / \mathrm{mL}$ & 0 & GE \\
\hline & $\mathrm{pC}_{1 / m \mathrm{~m}}$ & 0 & GE \\
\hline & $\mathrm{pCi} / \mathrm{mL}$ & 0 & TM \\
\hline & $p C i / m L$ & 0 & TM \\
\hline
\end{tabular}

$\overline{- \text { = exceeded holding time. }} \mathbf{a}=$ exceeded final primary drinking water standard. 


\section{WELL KAC 5}

$\begin{array}{lllllll}\text { SRS Coord. Lat/Longitude } & \text { Screen Zone Elevation } & \text { Top of Casing } & \text { Casing } & \text { Pump Formation } \\ \text { N53161.7 } & 33.213047^{\circ} \mathrm{N} & 224.3-204.3 \mathrm{ft} \mathrm{msl} & 259 \mathrm{ft} \mathrm{msl} & 4 \text { " PVC } & \text { S } & \text { Water table } \\ \text { E42716.3 } & 81.657589^{\circ} \mathrm{W} & & & & \end{array}$

\section{FIELD MEASUREMENTS}

Sample date: 02/04/93

Depth to water: $36.39 \mathrm{ft}(11.09 \mathrm{~m})$ below TOC

Water elevation: $222.61 \mathrm{ft}(67.85 \mathrm{~m}) \mathrm{msl}$

Sp. conductance: $61 \mu \mathrm{S} / \mathrm{cm}$

Water evacuated before sampling: $46 \mathrm{gal}$
Time: $12: 20$

$\mathrm{pH}: 5.6$

Alkalinity: $3 \mathrm{mg} / \mathrm{L}$

Water temperature: $19.5^{\circ} \mathrm{C}$

Volumes purged: 3.8 well volumes

LABORATORY ANALYSES

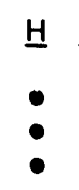

\section{Analyte}

$\mathrm{pH}$

Specific conductance

Turbidity

Aluminum

Arsenic

Barium

Cadmium

Calcium

Chloride

Chromium

2,4-Dichlorophenoxyacetic acid

Endrin

Fluoride

Iron

Lead

Lindane

Magnesium

Manganese

Mercury

Methoxychlor

Nitrate as nitrogen

Phenols

Potassium

Selenium

Silica

Silver

Sodium

Sulfate

Total dissolved solids

Total organic carbon

Total organic halogens

Total phosphates (as P)

Toxaphene

2,4,5-TP (Silvex)

Gross atpha

Nonvolatile beta

Radium-226

Radium-228

Tritium

$\begin{aligned} & \text { Result } \\ & 5.3 \\ & 58 \\ & 0.94 \\ & 30 \\ &<2.0 \\ & 4.1 \\ &<2.0 \\ & 326 \\ & 5.580 \\ &<4.0 \\ &<1.1 \\ &<0.11 \\ &<100 \\ & 176 \\ &<3.0 \\ &<0.055 \\ & 185 \\ & 11 \\ &<0.20 \\ &<0.55 \\ & 120 \\ &<5.0 \\ & 654 \\ &<2.0 \\ & 4.580 \\ &<2.0 \\ & 7.050 \\ & 8,860 \\ & 42.000 \\ &<1.000 \\ &<5.0 \\ &<50 \\ &<1.1 \\ &<0.54 \\ & 4.0 \mathrm{E}-01 \pm 5.0 \mathrm{E}-01 \\ & 8.0 \mathrm{E}-01 \pm 1.4 \mathrm{E}+00 \\ & 1.2 \mathrm{E}-01 \pm 1.2 \mathrm{E}-01 \\ &<3.0 \mathrm{E}-01 \\ & 6.8 \mathrm{E}-01 \pm 2.2 \mathrm{E}-01 \\ &\end{aligned}$

\begin{tabular}{|c|c|c|c|}
\hline Mod & Unit & Flag & $\underline{\text { Lab }}$ \\
\hline J & $\mathrm{pH}$ & 0 & WA \\
\hline $\mathrm{J}$ & $\mu \mathrm{S} / \mathrm{cm}$ & 0 & WA \\
\hline J & NTU & 0 & WA \\
\hline \multirow[t]{2}{*}{ J3 } & $\mu \mathrm{g} / \mathrm{L}$ & 1 & WA \\
\hline & $\mu \mathrm{g} / \mathrm{L}$ & 0 & WA \\
\hline \multirow[t]{2}{*}{ J3 } & $\mu \mathrm{g} / \mathrm{L}$ & 0 & WA \\
\hline & $\mu \mathrm{g} / \mathrm{L}$ & 0 & WA \\
\hline \multirow[t]{6}{*}{ v } & $\mu \mathrm{g} / \mathrm{L}$ & 0 & WA \\
\hline & $\mu \mathrm{g} / \mathrm{L}$ & 0 & WA \\
\hline & $\mu \mathrm{g} / \mathrm{L}$ & 0 & WA \\
\hline & $\mu \mathrm{g} / \mathrm{L}$ & 0 & WA \\
\hline & $\mu \mathrm{g} / \mathrm{L}$ & 0 & WA \\
\hline & $\mu \mathrm{g} / \mathrm{L}$ & 0 & WA \\
\hline \multirow[t]{9}{*}{ v } & $\mu \mathrm{g} / \mathrm{L}$ & 1 & WA \\
\hline & $\mu \mathrm{g} / \mathrm{L}$ & 0 & WA \\
\hline & $\mu \mathrm{g} / \mathrm{L}$ & 0 & WA \\
\hline & $\mu \mathrm{g} / \mathrm{L}$ & 0 & WA \\
\hline & $\mu \mathrm{g} / \mathrm{L}$ & 0 & WA \\
\hline & $\mu \mathrm{g} / \mathrm{L}$ & 0 & WA \\
\hline & $\mu g / L$ & 0 & WA \\
\hline & $\mu \mathrm{g} / \mathrm{L}$ & 0 & WA \\
\hline & $\mu \mathrm{g} / \mathrm{L}$ & 0 & WA \\
\hline \multirow{4}{*}{ J3 } & $\mu \mathrm{g} / \mathrm{L}$ & 0 & WA \\
\hline & $\mu \mathrm{g} / \mathrm{L}$ & 0 & WA \\
\hline & $\mu \mathrm{g} / \mathrm{L}$ & 0 & WA \\
\hline & $\mu \mathrm{g} / \mathrm{L}$ & 0 & WA \\
\hline \multirow{13}{*}{ V } & $\mu \mathrm{g} / \mathrm{L}$ & 0 & WA \\
\hline & $\mu \mathrm{g} / \mathrm{L}$ & 0 & WA \\
\hline & $\mu \mathrm{g} / \mathrm{L}$ & 0 & WA \\
\hline & $\mu \mathrm{g} / \mathrm{L}$ & 0 & WA \\
\hline & $\mu \mathrm{g} / \mathrm{L}$ & 0 & WA \\
\hline & $\mu \mathrm{g} / \mathrm{L}$ & 0 & WA \\
\hline & $\mu \mathrm{g} / \mathrm{L}$ & 0 & WA \\
\hline & $\mu \mathrm{g} / \mathrm{L}$ & 0 & WA \\
\hline & $p C i / L$ & 0 & TM \\
\hline & $\mathrm{pCi} / \mathrm{L}$ & 0 & TM \\
\hline & $\mathrm{pCi} / \mathrm{L}$ & 0 & TM \\
\hline & $\mathrm{pCi} / \mathrm{L}$ & 0 & TM \\
\hline & $p \mathrm{Ci} / \mathrm{mL}$ & 0 & TM \\
\hline
\end{tabular}

- exceeded holding time. = exceeded final primary drinking water standard. 
WSRC-TR-93-227

\section{WELL KAC 6}

\begin{tabular}{|c|c|c|c|c|c|c|}
\hline SRS Coord. & Lat/Longitude & Screen Zone Elevation & Top of Casing & Casing & Puing & Formation \\
\hline $\begin{array}{l}\text { N53139.9 } \\
\text { E42693.5 }\end{array}$ & $\begin{array}{l}33.212962^{\circ} \circ \mathrm{N} \\
81.657606^{\circ} \mathrm{W}\end{array}$ & $224.6-204.6 \mathrm{ft} \mathrm{msl}$ & $259 \mathrm{ft} \mathrm{msl}$ & 4" PVC & $S$ & Water table \\
\hline
\end{tabular}

\section{FIELD MEASUREMENTS}

Sample date: 02/05/93

Depth to water: $36.41 \mathrm{ft}(11.10 \mathrm{~m})$ below TOC

Water elevation: $222.59 \mathrm{ft}(67.85 \mathrm{~m}) \mathrm{ms}$

Sp. conductance: $107 \mu \mathrm{S} / \mathrm{cm}$

Water evacuated before sampling: $10 \mathrm{gal}$

The well went dry during purging.

\section{LABORATORY ANALYSES}

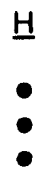

H

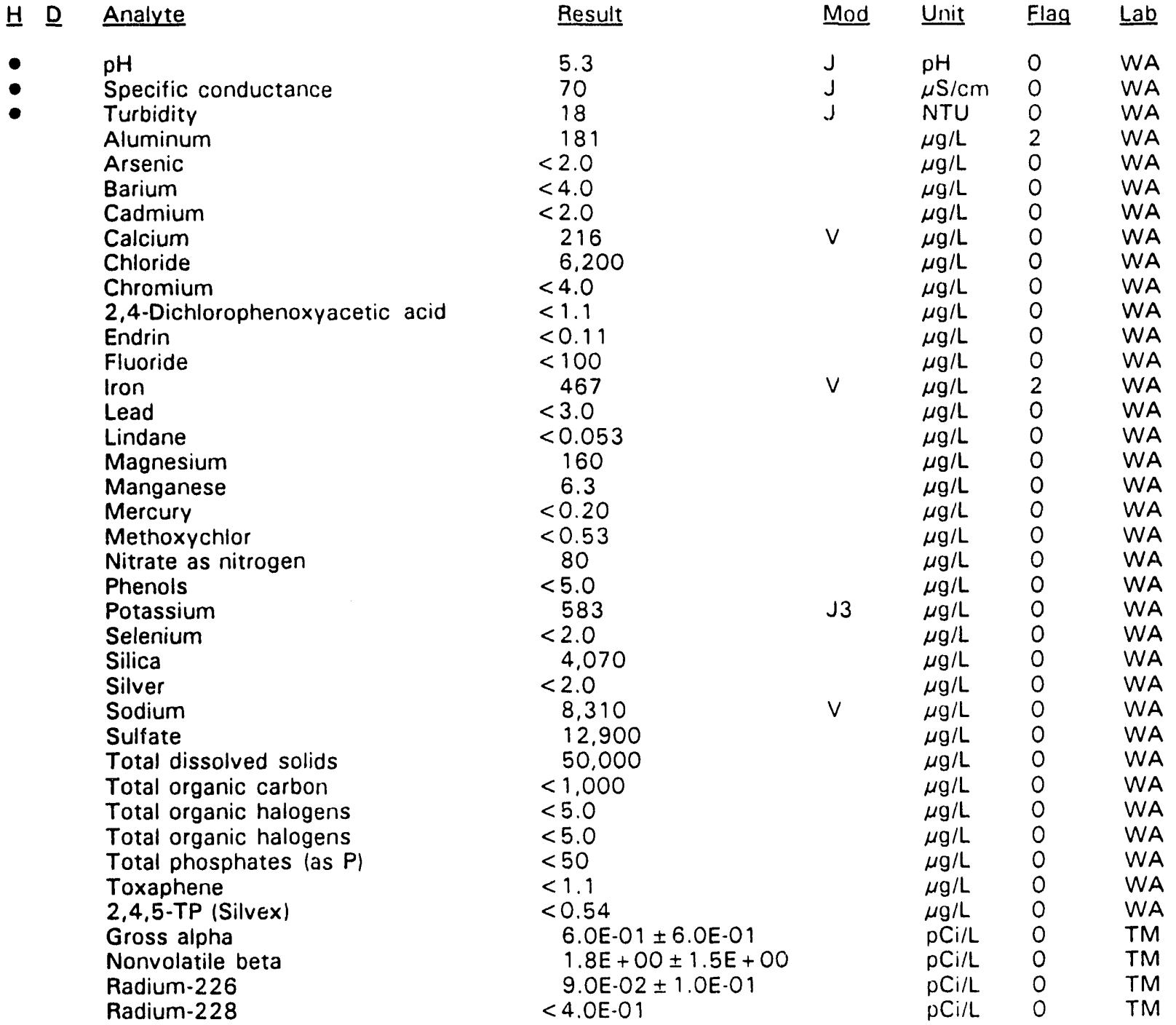

Time: $9: 15$

pH: 5.3

Alkalinity: $5 \mathrm{mg} / \mathrm{L}$

Water temperature: $17.2{ }^{\circ} \mathrm{C}$

Volumes purged: 0.8 well volumes

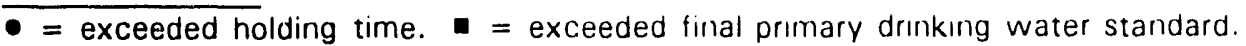


WSRC-TR-93-227

WELL KAC 6 collected on 02/05/93, laboratory analyses (cont.)

H $\underline{H} \begin{array}{llll}\text { Analyte } & \underline{\text { Result }} & \text { Mod Unit } & \underline{\text { Flag }} \underline{\text { Lab }} \\ \text { Tritium } & 8.1 E-01 \pm 2.7 E-01 & \text { pCi/mL } 0 & T M\end{array}$

\section{WELL KAC 7}

\begin{tabular}{|c|c|c|c|c|c|c|}
\hline SRS Coord. & Lat/Longitude & Screen Zone Elevation & Top of Casing & Casing & Pump & Formation \\
\hline $\begin{array}{l}\text { N53252.9 } \\
\text { E42574.5 }\end{array}$ & $\begin{array}{l}33.213018^{\circ} \mathrm{N} \\
81.658139^{\circ} \mathrm{W}\end{array}$ & $223.0-203.0 \mathrm{ft} \mathrm{msl}$ & $265.1 \mathrm{ft} \mathrm{msl}$ & 4" PVC & $\mathrm{s}$ & Water table \\
\hline
\end{tabular}

\section{FIELD MEASUREMENTS}

Sample date: 02/05/93

Depth to water: $45.61 \mathrm{ft}(13.90 \mathrm{~m})$ below TOC

Water elevation: $219.49 \mathrm{ft}(66.90 \mathrm{~m}) \mathrm{ms}$ l

Sp. conductance: $368 \mu \mathrm{S} / \mathrm{cm}$

Water evacuated before sampling: $8 \mathrm{gal}$

The well went dry during purging.

\section{LABORATORY ANALYSES}

H

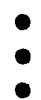

\begin{tabular}{|c|c|c|c|c|c|}
\hline Analvte & Result & Mod & Unit & Flag & $\underline{\mathrm{Lab}}$ \\
\hline $\mathrm{pH}$ & 5.6 & $J$ & $\rho H$ & 0 & WA \\
\hline Specific conductance & 310 & $J$ & $\mu \mathrm{S} / \mathrm{cm}$ & 1 & WA \\
\hline Turbidity & 16 & $J$ & NTU & 0 & WA \\
\hline Aluminum & 4,660 & & $\mu \mathrm{g} / \mathrm{L}$ & 2 & WA \\
\hline Arsenic & $<2.0$ & & $\mu \mathrm{g} / \mathrm{L}$ & 0 & WA \\
\hline Barium & 4.3 & J3 & $\mu \mathrm{g} / \mathrm{L}$ & 0 & WA \\
\hline Cadmium & $<2.0$ & & $\mu \mathrm{g} / \mathrm{L}$ & 0 & WA \\
\hline Calcium & 1,120 & V & $\mu \mathrm{g} / \mathrm{L}$ & 0 & WA \\
\hline Chloride & 7.940 & & $\mu \mathrm{g} / \mathrm{L}$ & 0 & WA \\
\hline Chromium & $<4.0$ & & $\mu \mathrm{g} / \mathrm{L}$ & 0 & WA \\
\hline 2,4-Dichlorophenoxyacetic acid & $<1.1$ & & $\mu \mathrm{g} / \mathrm{L}$ & 0 & WA \\
\hline Endrin & $<0.11$ & & $\mu \mathrm{g} / \mathrm{L}$ & 0 & WA \\
\hline Fluoride & $<100$ & & $\mu \mathrm{g} / \mathrm{L}$ & 0 & WA \\
\hline Iron & 987 & $\mathrm{~V}$ & $\mu g / L$ & 2 & WA \\
\hline Lead & 42 & & $\mu \mathrm{g} / \mathrm{L}$ & 2 & WA \\
\hline Lindane & $<0.054$ & & $\mu \mathrm{g} / \mathrm{L}$ & 0 & WA \\
\hline Magnesium & 147 & & $\mu \mathrm{g} / \mathrm{L}$ & 0 & WA \\
\hline Manganese & 17 & & $\mu \mathrm{g} / \mathrm{L}$ & 0 & WA \\
\hline Mercury & $<0.20$ & & $\mu \mathrm{g} / \mathrm{L}$ & 0 & WA \\
\hline Methoxychlor & $<0.54$ & & $\mu \mathrm{g} / \mathrm{L}$ & 0 & WA \\
\hline Nitrate as nitrogen & 1.160 & & $\mu \mathrm{g} / \mathrm{L}$ & 0 & WA \\
\hline Phenols & $<5.0$ & & $\mu \mathrm{g} / \mathrm{L}$ & 0 & WA \\
\hline Potassium & 519 & J3 & $\mu \mathrm{g} / \mathrm{L}$ & 0 & WA \\
\hline Selenium & $<2.0$ & & $\mu \mathrm{g} / \mathrm{L}$ & 0 & WA \\
\hline Silica & 3,630 & & $\mu \mathrm{g} / \mathrm{L}$ & 0 & WA \\
\hline Silver & $<2.0$ & & $\mu \mathrm{g} / \mathrm{L}$ & 0 & WA \\
\hline Sodium & 43,400 & V & $\mu \mathrm{g} / \mathrm{L}$ & 0 & WA \\
\hline Sulfate & 90,000 & & $\mu g / L$ & 0 & WA \\
\hline Total dissolved solids & 189,000 & & $\mu \mathrm{g} / \mathrm{L}$ & 0 & WA \\
\hline Total organic carbon & $<1,000$ & & $\mu \mathrm{g} / \mathrm{L}$ & 0 & WA \\
\hline Total organic halogens & $<5.0$ & & $\mu \mathrm{g} / \mathrm{L}$ & 0 & WA \\
\hline Total phosphates $\{$ as $P$ \} & $<50$ & & $\mu \mathrm{g} / \mathrm{L}$ & 0 & WA \\
\hline Toxaphene & $<1.1$ & & $\mu \mathrm{g} / \mathrm{L}$ & 0 & WA \\
\hline
\end{tabular}

\begin{tabular}{|c|c|c|c|c|c|}
\hline Analvte & Result & Mod & Unit & Flag & $\underline{\mathrm{Lab}}$ \\
\hline $\mathrm{pH}$ & 5.6 & $J$ & $\rho H$ & 0 & WA \\
\hline Specific conductance & 310 & $J$ & $\mu \mathrm{S} / \mathrm{cm}$ & 1 & WA \\
\hline Turbidity & 16 & $J$ & NTU & 0 & WA \\
\hline Aluminum & 4,660 & & $\mu \mathrm{g} / \mathrm{L}$ & 2 & WA \\
\hline Arsenic & $<2.0$ & & $\mu \mathrm{g} / \mathrm{L}$ & 0 & WA \\
\hline Barium & 4.3 & J3 & $\mu \mathrm{g} / \mathrm{L}$ & 0 & WA \\
\hline Cadmium & $<2.0$ & & $\mu \mathrm{g} / \mathrm{L}$ & 0 & WA \\
\hline Calcium & 1,120 & V & $\mu \mathrm{g} / \mathrm{L}$ & 0 & WA \\
\hline Chloride & 7.940 & & $\mu \mathrm{g} / \mathrm{L}$ & 0 & WA \\
\hline Chromium & $<4.0$ & & $\mu \mathrm{g} / \mathrm{L}$ & 0 & WA \\
\hline 2,4-Dichlorophenoxyacetic acid & $<1.1$ & & $\mu \mathrm{g} / \mathrm{L}$ & 0 & WA \\
\hline Endrin & $<0.11$ & & $\mu \mathrm{g} / \mathrm{L}$ & 0 & WA \\
\hline Fluoride & $<100$ & & $\mu \mathrm{g} / \mathrm{L}$ & 0 & WA \\
\hline Iron & 987 & $\mathrm{~V}$ & $\mu g / L$ & 2 & WA \\
\hline Lead & 42 & & $\mu \mathrm{g} / \mathrm{L}$ & 2 & WA \\
\hline Lindane & $<0.054$ & & $\mu \mathrm{g} / \mathrm{L}$ & 0 & WA \\
\hline Magnesium & 147 & & $\mu \mathrm{g} / \mathrm{L}$ & 0 & WA \\
\hline Manganese & 17 & & $\mu \mathrm{g} / \mathrm{L}$ & 0 & WA \\
\hline Mercury & $<0.20$ & & $\mu \mathrm{g} / \mathrm{L}$ & 0 & WA \\
\hline Methoxychlor & $<0.54$ & & $\mu \mathrm{g} / \mathrm{L}$ & 0 & WA \\
\hline Nitrate as nitrogen & 1.160 & & $\mu \mathrm{g} / \mathrm{L}$ & 0 & WA \\
\hline Phenols & $<5.0$ & & $\mu \mathrm{g} / \mathrm{L}$ & 0 & WA \\
\hline Potassium & 519 & J3 & $\mu \mathrm{g} / \mathrm{L}$ & 0 & WA \\
\hline Selenium & $<2.0$ & & $\mu \mathrm{g} / \mathrm{L}$ & 0 & WA \\
\hline Silica & 3,630 & & $\mu \mathrm{g} / \mathrm{L}$ & 0 & WA \\
\hline Silver & $<2.0$ & & $\mu \mathrm{g} / \mathrm{L}$ & 0 & WA \\
\hline Sodium & 43,400 & V & $\mu \mathrm{g} / \mathrm{L}$ & 0 & WA \\
\hline Sulfate & 90,000 & & $\mu g / L$ & 0 & WA \\
\hline Total dissolved solids & 189,000 & & $\mu \mathrm{g} / \mathrm{L}$ & 0 & WA \\
\hline Total organic carbon & $<1,000$ & & $\mu \mathrm{g} / \mathrm{L}$ & 0 & WA \\
\hline Total organic halogens & $<5.0$ & & $\mu \mathrm{g} / \mathrm{L}$ & 0 & WA \\
\hline Total phosphates $\{$ as $P$ \} & $<50$ & & $\mu \mathrm{g} / \mathrm{L}$ & 0 & WA \\
\hline Toxaphene & $<1.1$ & & $\mu \mathrm{g} / \mathrm{L}$ & 0 & WA \\
\hline
\end{tabular}

Time: $9: 32$

$\mathrm{pH}: 5.5$

Alkalinity: $12 \mathrm{mg} / \mathrm{L}$

Water temperature: $16.5^{\circ} \mathrm{C}$

Volumes purged: 0.7 well volumes

\footnotetext{
$\overline{0}$ = exceeded holding time. $\mathbf{a}=$ exceeded final primary drinking water standard.
} 
WELL KAC 7 collected on 02/05/93, laboratory analyses (cont.)

H D Analyte

$2,4,5-T P$ (Silvex)
Gross alpha
Nonvolatile beta
Radium-226
Radium-228
Tritium

Result

$$
\begin{aligned}
< & 0.54 \\
& 2.3 E+00 \pm 1.1 E+00 \\
& 2.5 E+00 \pm 1.7 E+00 \\
& 2.4 E-01 \pm 1.7 E \cdot 01 \\
< & 3.0 E-01 \\
& 8.6 E+00 \pm 2.9 E+00
\end{aligned}
$$

\begin{tabular}{|c|c|c|c|c|c|c|}
\hline SRS Coord. & Lat/Longitude & Screen Zone Elevation & Top of Casing & Casing & Puinp & Formation \\
\hline $\begin{array}{l}\text { N53136.0 } \\
\text { E42641.9 }\end{array}$ & $\begin{array}{l}33.212869 \circ \mathrm{N} \\
81.657734{ }^{\circ} \mathrm{W}\end{array}$ & $217.1-197.1 \mathrm{ft} \mathrm{msl}$ & $267.1 \mathrm{ft} \mathrm{msl}$ & 4" PVC & $\mathrm{S}$ & Water table \\
\hline
\end{tabular}

$\begin{array}{llll}\text { Mod Unit } & \text { Flag } & \text { Lab } \\ \mu \mathrm{g} / \mathrm{L} & 0 & \text { WA } \\ \mathrm{pCi} / \mathrm{L} & 0 & \text { TM } \\ \mathrm{pCi} / \mathrm{L} & 0 & \text { TM } \\ \mathrm{pCi} / \mathrm{L} & 0 & \text { TM } \\ \mathrm{pCi} / \mathrm{L} & 0 & \mathrm{TM} \\ \mathrm{pCi} / \mathrm{mL} & 0 & \mathrm{TM}\end{array}$

WELL KAC 8

FIELD MEASUREMENTS

Sample date: 02/04/93

Depth to water: $42.57 \mathrm{ft}(12.98 \mathrm{~m})$ below TOC

Water elevation: $224.53 \mathrm{ft}(68.44 \mathrm{~m}) \mathrm{msl}$

Sp. conductance: $269 \mu \mathrm{S} / \mathrm{cm}$

Water evacuated before sampling: $68 \mathrm{gal}$
Time: $12: 52$

\begin{tabular}{|c|c|c|c|c|c|c|}
\hline$\underline{H} \quad \underline{D}$ & Analyte & Result & Mod & $\underline{\text { Unit }}$ & Flag & $\underline{\text { Lab }}$ \\
\hline - & $\mathrm{pH}$ & 5.6 & J & $\mathrm{pH}$ & 0 & WA \\
\hline & Specific conductance & 271 & $\mathrm{~J}$ & $\mu \mathrm{S} / \mathrm{cm}$ & 1 & WA \\
\hline & Turbidity & 18 & $\mathrm{~J}$ & NTU & 0 & WA \\
\hline & Aluminum & 101 & & $\mu \mathrm{g} / \mathrm{L}$ & 2 & WA \\
\hline & Arsenic & $<2.0$ & & $\mu \mathrm{g} / \mathrm{L}$ & 0 & WA \\
\hline & Barium & 12 & & $\mu \mathrm{g} / \mathrm{L}$ & 0 & WA \\
\hline & Benzene & $<5.0$ & & $\mu \mathrm{g} / \mathrm{L}$ & 0 & WA \\
\hline & Bromodichloromethane & $<5.0$ & & $\mu \mathrm{g} / \mathrm{L}$ & 0 & WA \\
\hline & Bromoform & $<5.0$ & & $\mu \mathrm{g} / \mathrm{L}$ & 0 & WA \\
\hline & Bromomethane (Methyl bromide) & $<10$ & & $\mu \mathrm{g} / \mathrm{L}$ & 0 & WA \\
\hline & Cadmium & $<2.0$ & & $\mu \mathrm{g} / \mathrm{L}$ & 0 & WA \\
\hline & Calcium & 852 & $\mathrm{v}$ & $\mu \mathrm{g} / \mathrm{L}$ & 0 & WA \\
\hline & Carbon tetrachloride & $<5.0$ & & $\mu \mathrm{g} / \mathrm{L}$ & 0 & WA \\
\hline & Chloride & 9,890 & & $\mu \mathrm{g} / \mathrm{L}$ & 0 & WA \\
\hline & Chlorobenzene & $<5.0$ & & $\mu \mathrm{g} / \mathrm{L}$ & 0 & WA \\
\hline & Chloroethane & $<10$ & & $\mu \mathrm{g} / \mathrm{L}$ & 0 & WA \\
\hline & Chloroethene (Vinyl chloride) & $<10$ & & $\mu \mathrm{g} / \mathrm{L}$ & 0 & WA \\
\hline & 2-Chloroethyl vinyl ether & $<10$ & & $\mu \mathrm{g} / \mathrm{L}$ & 0 & WA \\
\hline & Chloroform & 2.0 & $\mathrm{~J}$ & $\mu g / L$ & 0 & WA \\
\hline & Chloromethane (Methyl chloride) & 1.3 & J & $\mu \mathrm{g} / \mathrm{L}$ & 0 & WA \\
\hline & Chromium & 4.5 & J3 & $\mu \mathrm{g} / \mathrm{L}$ & 0 & WA \\
\hline & Dibromochloromethane & $<5.0$ & & $\mu \mathrm{g} / \mathrm{L}$ & 0 & WA \\
\hline & 1,1-Dichloroethane & $<5.0$ & & $\mu \mathrm{g} / \mathrm{L}$ & 0 & WA \\
\hline & 1,2-Dichloroethane & $<5.0$ & & $\mu \mathrm{g} / \mathrm{L}$ & 0 & WA \\
\hline & 1,1 -Dichloroethylene & $<5.0$ & & $\mu \mathrm{g} / \mathrm{L}$ & 0 & WA \\
\hline & trans-1,2-Dichloroethylene & $<5.0$ & & $\mu \mathrm{g} / \mathrm{L}$ & 0 & WA \\
\hline & Dichloromethane (Methylene chloride) & $<5.0$ & & $\mu \mathrm{g} / \mathrm{L}$ & 0 & WA \\
\hline & 2,4-Dichlorophenoxyacetic acid & $<1.1$ & & $\mu \mathrm{g} / \mathrm{L}$ & 0 & WA \\
\hline
\end{tabular}

$\mathrm{pH}: 5.4$

Alkalimity: $7 \mathrm{mg} / \mathrm{L}$

Water temperature: $18.9^{\circ} \mathrm{C}$

Volumes purged: 3.8 well volumes

\section{LABORATORY ANALYSES}

\footnotetext{
- =xceeded holding time. = exceeded final primary drinking water standard
} 
WELL KAC 8 collected on 02/04/93, laboratory analyses (cont.)

H D Analyte

1,2-Dichloropropane cis-1,3-Dichloropropene

trans-1,3-Dichloropropene

Endrin

Ethylbenzene

Fluoride

Iron

Lead

Lindane

Magnesium

Manganese

Mercury

Methoxychlor

Nitrate as nitrogen

Phenols

Potassium

Selenium

Silica

Silver

Sodium

Sulfate

1,1,2,2-Tetrachloroethane

Tetrachloroethylene

Toluene

Total dissolved solids

Total organic carbon

Total organic halogens

Total phosphates (as P)

Toxaphene

2,4,5-TP (Silvex)

1,1,1-Trichloroethane

1,1,2-Trichloroethane

Trichloroethylene

Trichlorofluoromethane

Gross alpha

Nonvolatile beta

Radium-226

Radium-228

Tritium
Result

$<5.0$

$<5.0$

$<5.0$

$<0.11$

$<5.0$

$<100$

475

$<3.0$

$<0.054$

490

36

$<0.20$

$<0.54$

145

$<5.0$

626

$<2.0$

4,340

$<2.0$

33,600

89,600

$<5.0$

$<5.0$

$<5.0$

160,000

$<1,000$

6.0

$<50$

$<1.1$

$<0.54$

$<5.0$

$<5.0$

$<5.0$

$<5.0$

$2.9 E+00 \pm 1.1 E+00$

$2.4 E+00 \pm 1.6 E+O 0$

$1.1 \mathrm{E}+00 \pm 3.9 \mathrm{E}-01$

$6.0 \mathrm{E}-01 \pm 9.0 \mathrm{E}-01$

$9.5 \mathrm{E}-01 \pm 3.0 \mathrm{E}-01$ $\underline{\text { Mod Unit }}$ Flag Lab

$\mu \mathrm{g} / \mathrm{L} \quad 0 \quad W A$

$\mu g / L \quad 0 \quad$ WA

$\mu g / L \quad 0 \quad W A$

$\mu \mathrm{g} / \mathrm{L} \quad 0 \quad W A$

$\mu \mathrm{g} / \mathrm{L} \quad 0 \quad$ WA

$\begin{array}{lll}\mu g / L & 0 & W A\end{array}$

$\begin{array}{llll}V & \mu g / L & 2 & W A\end{array}$

$\mu g / L \quad 0 \quad W A$

$\mu \mathrm{g} / \mathrm{L} \quad 0 \quad$ WA

$\mu \mathrm{g} / \mathrm{L} \quad 0 \quad$ WA

$\mu \mathrm{g} / \mathrm{L} \quad 1 \quad$ WA

$\mu \mathrm{g} / \mathrm{L} \quad 0 \quad$ WA

$\mu g / L \quad 0 \quad W A$

$\mu \mathrm{g} / \mathrm{L} \quad 0 \quad$ WA

$\mu \mathrm{g} / \mathrm{L} \quad 0 \quad$ WA

J3 $\mu \mathrm{g} / \mathrm{L} \quad 0 \quad W A$

$\mu \mathrm{g} / \mathrm{L} \quad 0 \quad$ WA

$\mu g / L \quad 0 \quad W A$

$\mu \mathrm{g} / \mathrm{L} \quad 0 \quad$ WA

$\begin{array}{llll}V & \mu g / L & 0 & W A\end{array}$

$\mu \mathrm{g} / \mathrm{L} \quad 0 \quad$ WA

$\mu \mathrm{g} / \mathrm{L} \quad 0 \quad W A$

$\mu \mathrm{g} / \mathrm{L} \quad 0 \quad$ WA

$\mu g / L \quad 0$

$\mu g / L \quad 0 \quad W A$

$\mu \mathrm{g} / \mathrm{L} \quad 0 \quad W A$

$\mu \mathrm{g} / \mathrm{L} \quad 0 \quad$ WA

$\mu \mathrm{g} / \mathrm{L} \quad 0 \quad$ WA

$\mu \mathrm{g} / \mathrm{L} \quad 0 \quad W A$

$\mu \mathrm{g} / \mathrm{L} \quad 0 \quad$ WA

$\mu \mathrm{g} / \mathrm{L} \quad 0 \quad$ WA

$\mu g / L \quad 0 \quad$ WA

$\mu g / L \quad 0 \quad W A$

$\mu \mathrm{g} / \mathrm{L} \quad 0 \quad W A$

pCi/L $\quad 0 \quad$ TM

pCi/L 00 TM

pCill $\quad 0 \quad T M$

$p \mathrm{Ci} / \mathrm{L} \quad 0 \quad \mathrm{TM}$

$\mathrm{pCi} / \mathrm{mL} \quad 0 \quad \mathrm{TM}$

$\overline{0}$ = exceeded holding time. = exceeded final primary drinking water standard. 
WELL KAC 9

$\begin{array}{lllllll}\text { SRS Coord. } & \text { Lat/Longitude } & \text { Screen Zone Elevation } & \text { Top of Casing } & \text { Casing } & \text { Puinp } & \text { Formation } \\ \text { N53197.8 } & 33.212918^{\circ} \mathrm{N} & 210.9-190.9 \mathrm{ft} \mathrm{msl} & 262.2 \mathrm{ft} \mathrm{msl} & 4^{\prime \prime} \text { PVC } & \text { S } & \text { Water table } \\ \text { E42588.1 } & 81.657996^{\circ} \mathrm{W} & & & \end{array}$

\section{FIELD MEASUREMENTS}

Sample date: 02/04/93

Depth to water: $47.62 \mathrm{ft}(14.51 \mathrm{~m})$ below TOC

Water elevation: $214.58 \mathrm{ft}(65.40 \mathrm{~m}) \mathrm{msl}$

Sp. conductance: $919 \mu \mathrm{S} / \mathrm{cm}$

Water evacuated before sampling: $62 \mathrm{gal}$

LABORATORY ANALYSES

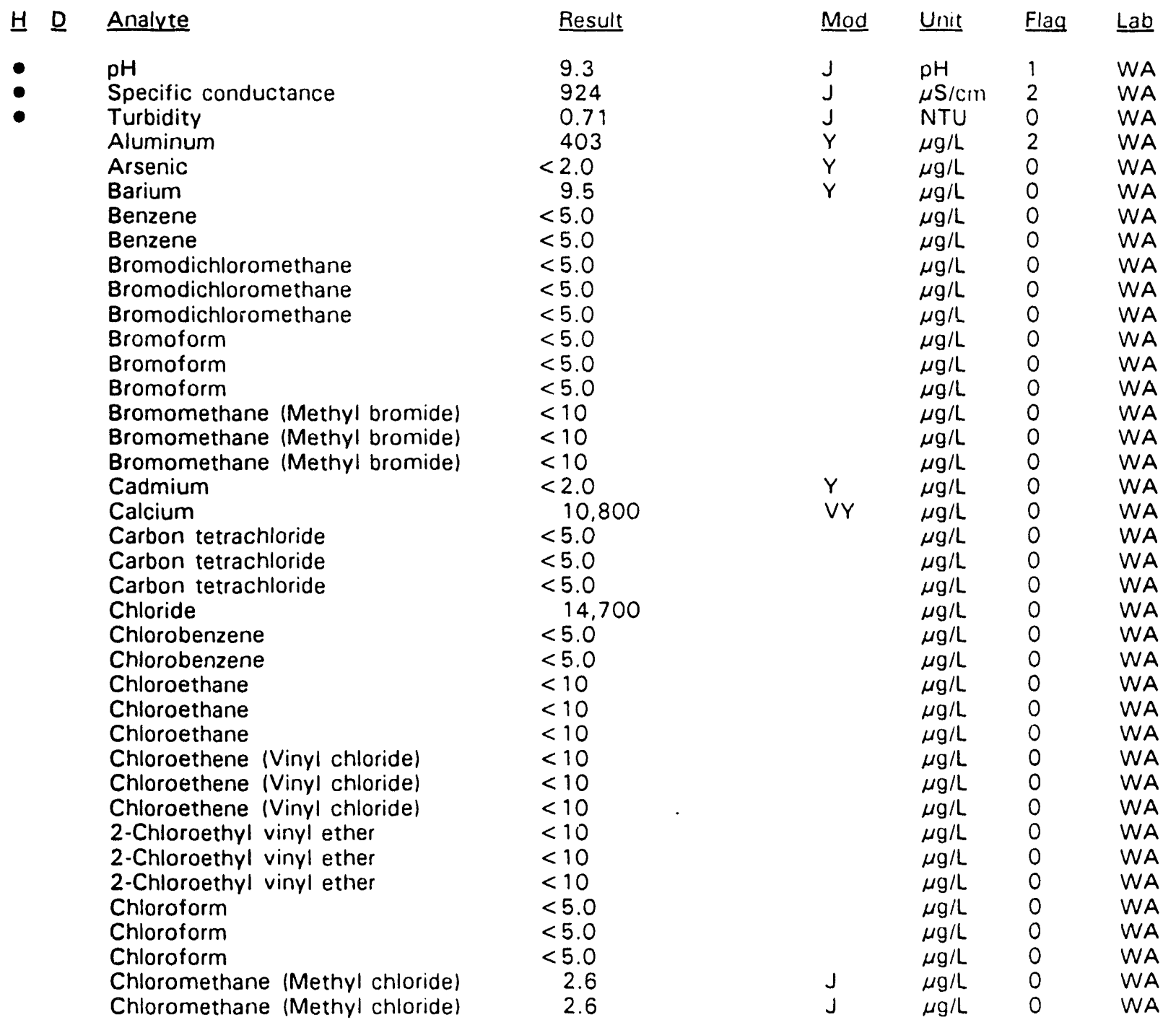

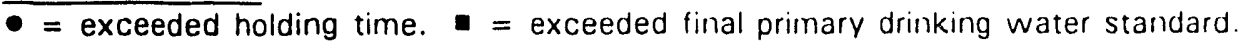

Time: $10: 35$

$\mathrm{pH}: 10.4$

Alkalinity: $72 \mathrm{mg} / \mathrm{L}$

Water temperature: $18.9^{\circ} \mathrm{C}$

Volumes purged: 4.0 well volumes 
WELi NAC 9 collected on $07.104 / 93$, laboratory analyses (cont.)

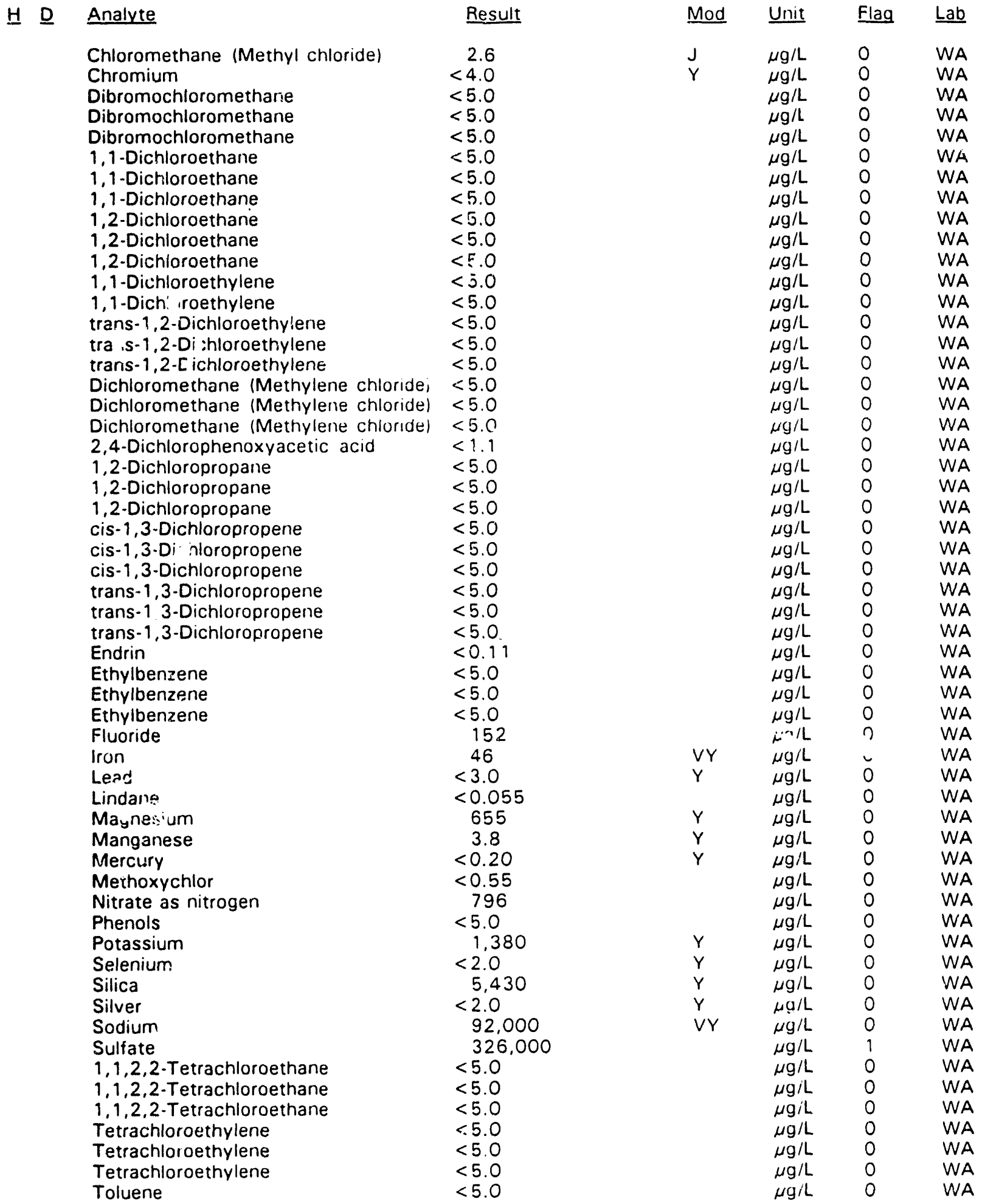

$\overline{-=\text { exceeded holding time. }}$ = exceeded final prinary drınkıng water standard. 
WELL KAC 9 collected on 02/04/93, laboratory anaiyses (cont.)

H. $\quad$ Analyte
Toluene
Total dissolved solids
Total organic carbon
Total organic halogens
Total phosphates (as P)
Toxaphene
2,4,5-TP (Silvex)
$1,1,1$-Trichloroethane
$1,1,1-$ Trichloroethane
1,1,1-Trichloroethane
$1,1,2-$ Trichloroethane
$1,1,2-$ Trichloroethane
1,1,2-Trichloroethane
Trichloroethylene
Trichloroethylene
Trichlorofluoromethane
Trichlorofluoromethane
Trichlorofluoromethane
Gross alpha
Nonvilatile beta
Radium-226
Radium-228
Tritium

$\begin{aligned} & \text { Result } \\ &<5.0 \\ & 604,000 \\ &<1.000 \\ & 15 \\ &<50 \\ &<1.1 \\ &<0.54 \\ &<5.0 \\ &<5.0 \\ &<5.0 \\ &<5.0 \\ &<5.0 \\ &<5.0 \\ &<5.0 \\ &<5.0 \\ &<5.0 \\ &<5.0 \\ &<5.0 \\ & 1.5 E+00 \pm 2.0 E+00 \\ & 6.4 E+00 \pm 4.5 E+00 \\ & 2.7 E-01 \pm 1.8 E-01 \\ & 4.0 E-01 \pm 8.0 E-01 \\ & 3.1 E+00 \pm 1.0 E+00\end{aligned}$

\footnotetext{
$\overline{0} \overline{0}$ exceeded holding time. = exceeded final primary drinkıng water standard
} 
WSRC-TR-93-277

\section{Appendix E - Data Quality/Useability Assessment}




\section{Data Quality/Useability Assessment}

Quality assurance/quality control (QA/QC) procedures relating to accuracy and precision of analyses performed on groundwater samples are followed in the field and laboratory and are reviewed prior to publication of results. The Environmental Protection Department/ Environmental Monitoring Section's (EPD/EMS) review of the volume of analytical data acquired each quarter and presented in various reports is an ongoing process; its review of the QA/QC data cannot be completed in time to meet the deadlines for the reports required by the Resource Conservation and Recovery Act and associated regulations. Other site and regulatory personnel can obtain further information on the data quality and useability in a variety of ways, including those described below.

\section{Data Qualification}

The contract laboratories continually assess their own accuracy and precision according to U.S. Environmental Protection Agency (EPA) guidelines. They submit sample- or batchspecific QA/QC information either at the same time as analytical results or in a quarterly summary. Properly defined and used result modifiers (also referred to as qualifiers) can be a key component in assessing data useability. Result modifiers designed by EPD/EMS and used by the primary laboratories are presented in Appendix D.

\section{Assessment of Accuracy of the Data}

Accuracy, or the nearness of the reported result to the true concentration of a constituent in a sample, can be assessed in several ways.

A laboratory's general accuracy can be judged by analysis of results obtained from known samples. The non-radionuclide contract laboratories analyze commercial reference samples every quarter at EPD/EMS' request. The results of these analyses are presented in the EPD/EMS quarterly report, The Savannah River Site's Groundwater Monitoring Program. The primary laboratories also seek or maintain state certification by participating periodically in performance studies; reference samples and analysis of results are provided by EPA. Results of these studies also are published in the EPD/EMS quarterly reports.

Analysis of blanks provides a tool for assessing the accuracy of both sampling and laboratory analysis. Results for all field blanks for the quarter can be found in the EPD/EMS quarterly reports. Any field or laboratory blanks that exceeded established minimums are identified in the same reports, in tables associating them with groundwater samples analyzed in the same batches.

Surrogates, organic compounds similar in chemical behavior to the compounds of interest but not normally found in environmental samples, are used to monitor the effect of the matrix on the accuracy of analyses for organic parameters. For example, for analyses of volatile organics by EPA Method 8240, three surrogate compounds are added to all samples 
and blanks in each analytical batch. In analyses of semivolatile organics, three to four acid compounds and three to four base/neutral compounds are used. Other surrogates are used in pesticides analyses. Percent recoveries for surrogate analyses are calculated by laboratory personnel, reported to EPD/EMS, reviewed, and entered into the database, but they are not published. If recoveries are not within specified limits, the laboratory is expected to re-run the samples or attach result qualifiers to the data identifying the anomalous results.

Sample-specific accuracy for both organic and inorganic parameters can be assessed by examination of matrix spike/matrix spike duplicate results. A sample is analyzed unspiked to determine a baseline set of values. A second portion of sample is spiked with known concentrations of compounds appropriate to the analyses being performed, typically 5 volatile organic compounds for volatile organics analyses, 11 semivolatile compounds for semivolatiles, 6 pesticide compounds for pesticides, all metals for metals analyses, and a known quantity of cyanide for cyanide analysis. The percentage of the spike compound that is recovered (i.e., measured in excess of the value obtained for the unspiked sample) is a direct measure of analytical accuracy. EPA requires matrix spike/matrix spike duplicates to be run at least once per 20 samples of similar matrix.

Matrix spike/matrix spike duplicate results are reported to EPD/EMS but are not published. For organic compounds, according to EPA guidelines, no action is taken on the basis of matrix spike/matrix spike duplicate data alone (i.e., no result modifiers are assigned solely on the basis of matrix spike results); however, the results can indicate if a lab is having a systematic problem in the analysis of one or more analytes.

In the case of inorganic compounds, such as metals, the matrix spike sample analysis provides information about the effect of each sample matrix on the digestion and measurement methodology. Data qualifiers can be assigned on the basis of the percentage of spike recovery and are reported in the published results tables.

\section{Assessment of Precision}

Precision of the analyses, or agreement of a set of replicate results among themselves, is assessed through the use of duplicates (laboratory-initiated) and blind replicates (provided by EPD/EMS). The results of duplicate and replicate analyses are presented in the results tables of the first, second, and third quarter reports. Duplicate and replicate results are not presented in fourth quarter reports; the results tables present instead only the highest result for each analyte for each quarter of the year.

The laboratories assess precision by calculating the relative percent difference, or RPD, for each pair of laboratory-initiated duplicate results. During 1992, at least one of the contract laboratories used a data qualifier (J3) to modify metals analyses when the RPD for laboratory duplicates was greater than $20 \%$.

Additional statistical comparisons of laboratory duplicate and blind replicate results, both intra- and interlaboratory, are presented in the EPD/EMS quarterly reports. The calculation used for these reports is the MRD, or mean relative difference, which is similar to EPA's RPD except that the ivRD provides a single value fur all of the analyses of a particular com- 
pound, either inter- or intralaboratory, during one quarter. Because detection limits may vary among samples, the MRD requires calculation of a reference detection limit, which is the detection limit at the 90th percentile of the array of limits in the population of all replicate and duplicate analyses for a given analyte during a particular quarter. The MRD is not method-specific.

\section{Method-Specific Accuracy and Precision}

The contract laboratories' EPA-approved laboratory procedures include QA/QC requirements as an integral part of the methods. Thus, knowledge of the method used in obtaining data is an important component of determining data useability. EPA has conducted extensive research and development on the methods approved for the analysis of water and waste water; information on the accuracy and precision of the method is available from EPA publications, as is full information on required QA/QC procedures. A listing of the methods used by the primary laboratories during first quarter 1992 is given below along with the source for the method description. Many, if not all, of these sources include presentations of representative accuracy and precision results.

\begin{tabular}{|c|c|c|}
\hline Method & Used to Analyze & Source \\
\hline EPA 120.1 & Specific conductance & EPA EMSL 1983 \\
\hline EPA150.1 & $\mathrm{pH}$ & EPA EMSL 1983 \\
\hline EPA 160.1 & Filterable residue (total dissolved solids) & EPA EMSL 1983 \\
\hline EPA 160.2 & Nonfilterable residue & EPA EMSL 1983 \\
\hline EPA 180.1 & Turbidity & EPA EMSL 1983 \\
\hline EPA200.7 & Trace elements & EPA EMSL 1983 \\
\hline EPA206.2 & Arsenic & EPA EMSL 1983 \\
\hline EPA208.2 & Barium & EPA EMSL 1983 \\
\hline EPA239.2 & Lead & EPA EMSL 1983 \\
\hline EPA245. 1 & Mercury & EPA EMSL 1983 \\
\hline EPA270.2 & Selenium & EPA EMSL 1983 \\
\hline EPA279.2 & Thallium & EPA EMSL 1983 \\
\hline EPA300.0 & Inorganics, non-metallics & EPA EMSL 1991 \\
\hline EPA310.1 & Alkalinity & EPA EMSL 1983 \\
\hline EPA325.2 & Chloride & EPA EMSL 1983 \\
\hline EPA335.3 & Cyanide & EPA EMSL 1983 \\
\hline EPA340.2 & Fluoride & EPA EMSL 1983 \\
\hline EPA353.1 & Nitrogen, nitrate-nitrite & EPA EMSL 1983 \\
\hline EPA353.2 & Nitrogen, nitrate, nitrite, or combined & EPA EMSL 1983 \\
\hline EPA353.3 & Nitrogen, nitrate-nitrite, or nitrite only & EPA EMSL 1983 \\
\hline EPA354.1 & Nitrogen, nitrite & EPA EMSL 1983 \\
\hline EPA365.1 & Phosphorus, all forms (reported as total phosphates) & EPA EMSL 1983 \\
\hline EPA365.2 & Phosphorus, all forms (reported as total phosphates) & EPA EMSL 1983 \\
\hline EPA375.4 & Sulfate, turbidimetric & EPA EMSL 1983 \\
\hline EPA376.2 & Sulfide & EPA EMSL 1983 \\
\hline APHA 403 & Alkalinity & APHA 1985 \\
\hline EPA413.1 & Oil \& grease & EPA EMSL 1983 \\
\hline APHA415A & lodine & APHA 1985 \\
\hline EPA4 15.1 & Total organic carbon & EPA EMSL 1983 \\
\hline EPA4 18.1 & Petroleum hydrocarbons & EPA EMSL 1983 \\
\hline EPA420.1 & Phenolics & EPA EMSL 1983 \\
\hline EPA420.2 & Phenolics & EPA EMSL 1983 \\
\hline APHATO5 & Tutal alpha-emiting radivin & APHA 1985 \\
\hline
\end{tabular}


Method

ASTMD3869C

APHA 5320

EPA6010

EPA7041

EPA7060

EPA7421

EPA7470

EPA7740

EPA7841

EPA8010

EPA8020

EPA8080

EPA8 140

EPA8150

EPA8240

EPA 8270

EPA8280

EPA9012

EPA9020

EPA9030
Used to Analyze

lodide
Dissolved organic halogen
Metals
Antimony
Arsenic
Lead
Mercury
Selenium
Thallium
Halogenated volatile organics
Aromatic volatile organics
Organochlorine pesticides and PCBs
Organophosphorus pesticides
Chlorinated herbicides
GCMS VOA
GCMS semivolatiles
Dioxins and furans
Total cyanide
Total organic halides
Sulfides

Source

ASTM 1992
APHA 1989
EPA 1986
EPA 1986
EPA 1986
EPA 1986
EPA 1986
EPA 1986
EPA 1986
EPA 1986
EPA 1986
EPA 1986
EPA 1986
EPA 1986
EPA 1986
EPA 1986
EPA 1986
EPA 1986
EPA 1986
EPA 1986

An example of the available method-specific QA/QC information is that for the analysis of metals by EPA Method 6010/200.7 (EPA, 1986/EPA EMSL, 1983). The primary laboratories, General Engineering Laboratories (GE) and Roy F. Weston, Inc. (Weston), use this inductively coupled plasma (ICP) atomic emission spectrometric method.

The following precision and accuracy data are based on the experience of seven laboratories that applied the ICP technique to acid-distilled water matrices that had been dosed with various metal concentrates. (Note: not all seven laboratories analyzed all 14 elements.) The references give results for samples having three concentration ranges; the results here are for samples having the lowest values, similar to actual groundwater results for SRS.

ICP Precision and Accuracy Data

\section{Element}

Aluminum

Arsenic

Beryllium

Cadmium

Chromium

Cobalt

Copper

iron

Lead

Manganese

Nickel

Selenium
True value $(\mu \mathrm{g} / \mathrm{L})$

60

22

20

2.5

10

20

11

20

24

15

30

6
Mean reported

value $(\mu \mathrm{g} / \mathrm{L})$

62

19

20

2.9

10

20

11

19

30

15

28

8.5
Mean percent

$\underline{\mathrm{RSD}}^{\mathrm{a}}$

33

23

9.8

16

18

4.1

40

15

32

6.7

11

42 


\begin{tabular}{|c|c|c|c|}
\hline Element & True value $(\mu \mathrm{g} / \mathrm{L})$ & $\begin{array}{l}\text { Mean reported } \\
\text { value }(\mu \mathrm{g} / \mathrm{L})\end{array}$ & $\begin{array}{l}\text { Mean percent } \\
\underline{\mathrm{RSD}}^{\mathrm{a}}\end{array}$ \\
\hline Vanadium & 70 & 69 & 2.9 \\
\hline Zinc & 16 & 19 & 45 \\
\hline
\end{tabular}

Note: In EPA (1986), the column heading is Mean Standard Deviation (\%).

a Relative standard deviation.

As another example, EPA Method 601/8010 (CFR, 1991/EPA, 1986) is used by both GE and Weston for analyses of halogenated volatile organics. In the presentation of the method in both references, the following table gives method-specific accuracy and precision as functions of concentration. Contract laboratories are expected to achieve or at least approach these limits.

Accuracy and Precision as Functions of Concentration for EPA Method 601/8010

Parameter
Bromodichloromethane
Bromoform
Bromomethane
Carbon tetrachloride
Chlorobenzene
Chloroethane
2-Chloroethyl vinyl ether
Chloroform
Chloromethane
Dibromochloromethane
1,2-Dichlorobenzene
1,3-Dichlorobenzene
1,4-Dichlorobenzene
1,1-Dichloroethane
1,2-Dichloroethane
1,1-Dichloroethene
trans-1,2-Dichloroethene
1,2-Dichloropropane
cis-1,3-Dichloropropene
trans-1,3-Dichloropropene
Methylene chloride
1,1,2,2-Tetrachlorethane
Tetrachloroethylene
1,1,1-Trichloroethane
1,1,2-Trichloroethane
Trichloroethylene
Trichlorofluoromethane
Vinyl chloride

a $X^{\prime}=$ expected recovery for one or more measurements of a sample containing a concentration of $C_{1}$ in $\mu \mathrm{g} / \mathrm{L}$.

b Expected single analyst standard deviation of measurements.

\begin{tabular}{|c|c|c|}
\hline $\begin{array}{l}\text { Accuracy as } \\
\text { recovery, } x^{\circ a}(\mu \mathrm{g} / \mathrm{L})\end{array}$ & 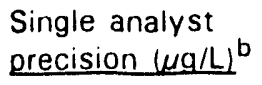 & $\begin{array}{l}\text { Overall } \\
\text { precision }(\mu \mathrm{g} / \mathrm{L})^{\mathrm{C}}\end{array}$ \\
\hline $1.12 C-1.02^{d}$ & $0.11 \bar{x}+0.04^{e}$ & $0.20 \bar{x}+1.00$ \\
\hline $0.96 C-2.05$ & $0.12 \bar{x}+0.58$ & $0.21 \bar{x}+2.41$ \\
\hline $0.76 C-1.27$ & $0.28 \bar{x}+0.27$ & $0.36 \bar{x}+0.94$ \\
\hline $0.98 C-1.04$ & $0.15 \bar{x}+0.38$ & $0.20 \bar{x}+0.39$ \\
\hline $1.00 C-1.23$ & $0.15 \bar{x}-0.02$ & $0.18 \bar{x}+1.21$ \\
\hline $0.99 C-1.53$ & $0.14 \bar{x}-0.13$ & $0.17 \bar{x}+0.63$ \\
\hline $1.00 \mathrm{C}$ & $0.20 \bar{x}$ & $0.35 \bar{x}$ \\
\hline $0.93 C-0.39$ & $0.13 \bar{x}+0.15$ & $0.19 \bar{x}-0.02$ \\
\hline $0.77 C+0.18$ & $0.28 \bar{x}-0.31$ & $0.52 \bar{x}+1.31$ \\
\hline $0.94 C+2.72$ & $0.11 \underline{\bar{x}}+1.10$ & $0.24 \underline{\bar{x}}+1.68$ \\
\hline $0.93 C+1.70$ & $0.20 \bar{x}+0.97$ & $0.13 \bar{x}+6.13$ \\
\hline $0.95 C+0.43$ & $0.14 \bar{x}+2.33$ & $0.26 \bar{x}+2.34$ \\
\hline $0.93 C-0.09$ & $0.15 \bar{x}+0.29$ & $0.20 \bar{x}+0.41$ \\
\hline $0.95 C-1.08$ & $0.09 \bar{x}+0.17$ & $0.14 \bar{x}+0.94$ \\
\hline $1.04 C-1.06$ & $0.11 \bar{x}+0.70$ & $0.15 \bar{x}+0.94$ \\
\hline $0.98 C-0.87$ & $0.21 \bar{x}-0.23$ & $0.29 \bar{x}-0.40$ \\
\hline $0.97 C-0.16$ & $0.11 \bar{x}+1.46$ & $0.17 \bar{x}+1.46$ \\
\hline $1.00 C$ & $0.13 \bar{x}$ & $0.23 \bar{x}$ \\
\hline $1.00 \mathrm{C}$ & $0.18 \bar{x}$ & $0.32 \bar{x}$ \\
\hline $1.00 \mathrm{C}$ & $0.18 \bar{x}$ & $0.32 \bar{x}$ \\
\hline $0.91 C-0.93$ & $0.11 \bar{x}+0.33$ & $0.21 \bar{x}+1.43$ \\
\hline $0.95 C+0.19$ & $0.14 \bar{X}+2.41$ & $0.23 \bar{x}+2.79$ \\
\hline $0.94 C+0.06$ & $0.14 \bar{x}+0.38$ & $0.18 \bar{x}+2.21$ \\
\hline $0.90 C-0.16$ & $0.15 \bar{x}+0.04$ & $0.20 \bar{x}+0.37$ \\
\hline $0.86 C+0.30$ & $0.13 \bar{x}-0.14$ & $0.19 \bar{x}+0.67$ \\
\hline $0.87 C+0.48$ & $0.13 \bar{x}-0.03$ & $0.23 \bar{x}+0.30$ \\
\hline $0.89 C-0.07$ & $0.15 \bar{x}+0.67$ & $0.26 \bar{x}+0.91$ \\
\hline $0.97 C-0.36$ & $0.13 \bar{x}+0.65$ & $0.27 \bar{x}+0.40$ \\
\hline
\end{tabular}


c Expected interlaboratory standard deviation of measurements.

d $C=$ true value for the concentration, in $\mu \mathrm{g} / \mathrm{L}$.

e $\bar{X}=$ average recovery found for measurements of samples containing a concentration of $C$, in $\mu \mathrm{g} / \mathrm{L}$.

$f$ Estimates based on performance in a single laboratory.

\section{References}

APHA (American Public Health Association), 1985. Standard Methods for the Examination of Water and Wastewater, 16th edition. Washington, DC.

APHA (American Public Health Association), 1989. Standard Methods for the Examination of Water and Wastewater, 17th edition. Washington, DC.

ASTM (American Society for Testing and Materials), 1992. 1992 Annual Book of ASTM Standards, Volume 11.02, Water (II). Philadelphia, PA.

CFR (Code of Federal Regulations), 1991. Guidelines Establishing Test Procedures for the Analysis of Pollutants, Title 40, Part 136, Appendix A. Revised July 1, 1991.

Washington, DC.

EPA (U.S. Environmental Protection Agency), 1986. Test Methods for Evaluating Solid Waste (SW-846), Volumes IA-IC. Washington, DC.

EPA (U.S. Environmental Protection Agency), 1987. Data Quality Objectives for Remedial Response Activities. PB88-131870; EPA/540/G-87/003. Washington, DC.

EPA (U.S. Environmental Protection Agency), 1988a. Contract Laboratory Program Statement of Work for Inorganics Analysis, Multi-Media, Multi-Concentration. SOW No. 788. Washington, DC.

EPA (U.S. Environmental Protection Agency), 1988b. Contract Laboratory Program Statement of Work for Organics Analysis Multi-Media, Multi-Concentration. SOW No. 288. Washington, DC.

EPA (U.S. Environmental Protection Agency), 1990. Guidance for Data Useability in Risk Assessment. Interim Final. EPA/540/G-90/008. Washington, DC.

EPA EMSL (U.S. Environmental Protection Agency, Environmental Monitoring and Systems Laboratory), 1979. Handbook for Analytical Quality Control in Water and Wastewater Laboratories. PB-297 451; EPA-600/4-79-019. Cincinnati, OH.

EPA EMSL (U.S. Environmental Protection Agency, Environmental Monitoring and Systems Laboratory), 1983. Methods for Chemical Analysis of Water and Wastes. Revised March 1983. Cincinnati, $\mathrm{OH}$.

EPA EMSL (U.S. Environmental Protection Agency, Environmental Monitoring and Systems Laboratory), 1991. Test Method, The Determination of Inorganic Anions in Water by Ion Chromatography-Methoa 300.0. Revised August 1991. Cincinnati, OH. 
WSRC.TR-93-277 

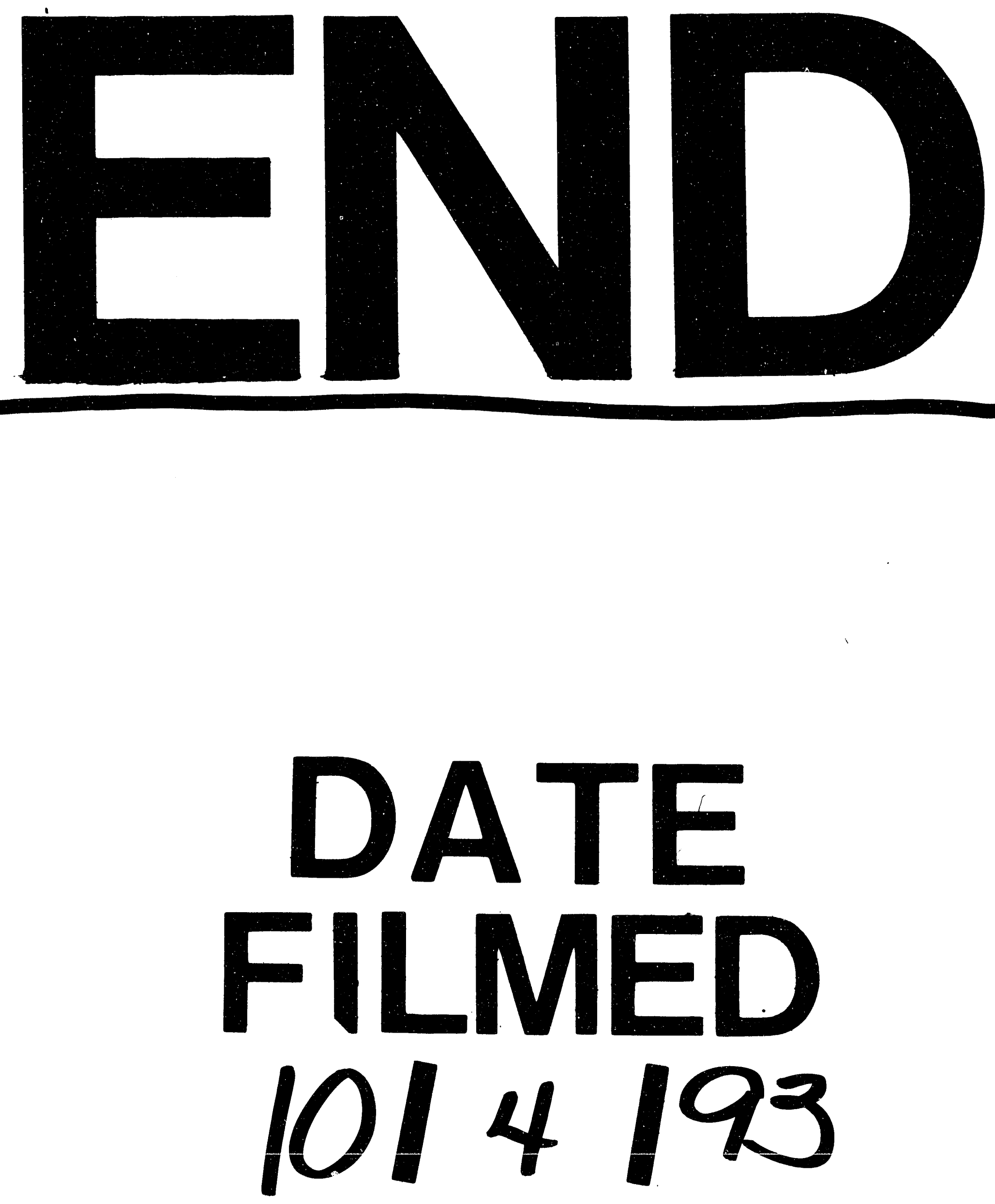
